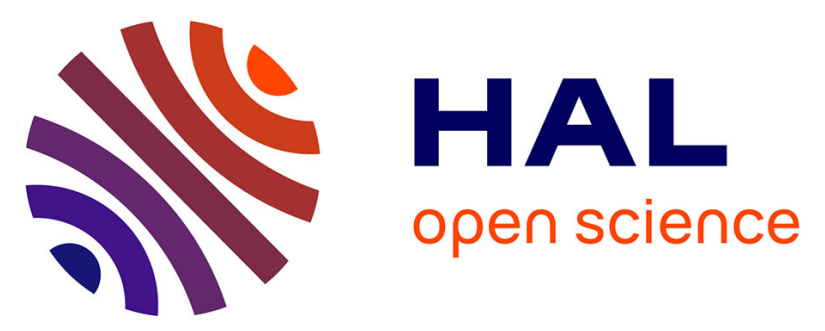

\title{
The INTEGRAL view of the pulsating hard X-ray sky: from accreting and transitional millisecond pulsars to rotation-powered pulsars and magnetars
}

A. Papitto, M. Falanga, W. Hermsen, S. Mereghetti, L. Kuiper, J. Poutanen, E. Bozzo, F. Ambrosino, F. Coti Zelati, V. de Falco, et al.

\section{To cite this version:}

A. Papitto, M. Falanga, W. Hermsen, S. Mereghetti, L. Kuiper, et al.. The INTEGRAL view of the pulsating hard X-ray sky: from accreting and transitional millisecond pulsars to rotation-powered pulsars and magnetars. New Astron.Rev., 2020, 91, pp.101544. 10.1016/j.newar.2020.101544 . hal03047487

\section{HAL Id: hal-03047487 \\ https://hal.science/hal-03047487}

Submitted on 4 Jan 2021

HAL is a multi-disciplinary open access archive for the deposit and dissemination of scientific research documents, whether they are published or not. The documents may come from teaching and research institutions in France or abroad, or from public or private research centers.
L'archive ouverte pluridisciplinaire HAL, est destinée au dépôt et à la diffusion de documents scientifiques de niveau recherche, publiés ou non, émanant des établissements d'enseignement et de recherche français ou étrangers, des laboratoires publics ou privés. 


\section{Journal Pre-proof}

The INTEGRAL view of the pulsating hard X-ray sky: from accreting and transitional millisecond pulsars to rotation-powered pulsars and magnetars

A. Papitto, M. Falanga, W. Hermsen, S. Mereghetti, L. Kuiper, J. Poutanen, E. Bozzo, F. Ambrosino, F. Coti Zelati, V. De Falco, D. de Martino, T. Di Salvo, P. Esposito, C. Ferrigno, M. Forot, D. Götz, C. Gouiffes, R. laria, P. Laurent, J. Li, Z. Li, T. Mineo, P. Moran, A. Neronov, A. Paizis, N. Rea, A. Riggio, A. Sanna, V. Savchenko, A. S lowikowska, A. Shearer, A. Tiengo, D.F. Torres

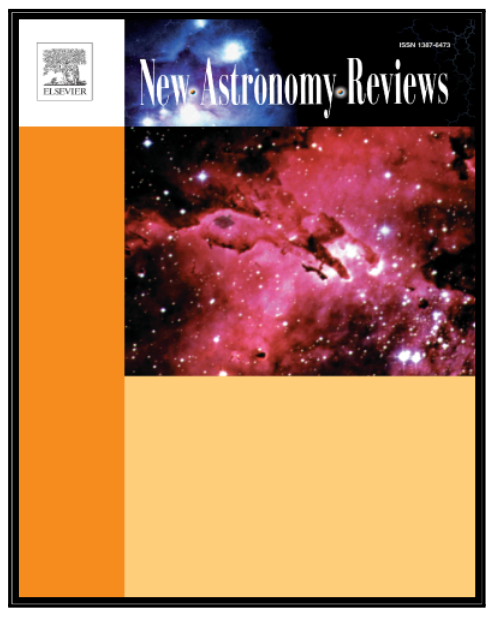

PII:

DOI:

Reference:

To appear in:

Received date:

Revised date:

Accepted date:
S1387-6473(20)30021-X https://doi.org/10.1016/j.newar.2020.101544 ASTREV 101544

New Astronomy Reviews

13 March 2020

9 August 2020

22 August 2020

Please cite this article as: A. Papitto, M. Falanga, W. Hermsen, S. Mereghetti, L. Kuiper, J. Poutanen, E. Bozzo, F. Ambrosino, F. Coti Zelati, V. De Falco, D. de Martino, T. Di Salvo, P. Esposito, C. Ferrigno, M. Forot, D. Götz, C. Gouiffes, R. laria, P. Laurent, J. Li, Z. Li, T. Mineo, P. Moran, A. Neronov, A. Paizis, N. Rea, A. Riggio, A. Sanna, V. Savchenko, A. S lowikowska, A. Shearer, A. Tiengo, D.F. Torres, The INTEGRAL view of the pulsating hard X-ray sky: from accreting and transitional millisecond pulsars to rotation-powered pulsars and magnetars, New Astronomy Reviews (2020), doi: https://doi.org/10.1016/j.newar.2020.101544

This is a PDF file of an article that has undergone enhancements after acceptance, such as the addition of a cover page and metadata, and formatting for readability, but it is not yet the definitive version of record. This version will undergo additional copyediting, typesetting and review before it is published in its final form, but we are providing this version to give early visibility of the article. Please note that, during the production process, errors may be discovered which could affect the content, and all legal disclaimers that apply to the journal pertain.

(C) 2020 Published by Elsevier B.V. 


\section{The INTEGRAL view of the pulsating hard X-ray sky: from accreting and transitional millisecond pulsars to rotation-powered pulsars and magnetars}

A. Papitto ${ }^{\mathrm{a}}$, M. Falanga ${ }^{\mathrm{b}}$, W. Hermsen ${ }^{\mathrm{c}, \mathrm{d}}$, S. Mereghetti ${ }^{\mathrm{e}}$, L. Kuiper $^{\mathrm{c}}$, J. Poutanen ${ }^{\mathrm{f}, \mathrm{g}, \mathrm{h}}$, E. Bozzo ${ }^{\mathrm{i}}$, F. Ambrosino ${ }^{\mathrm{j}}$, F. Coti Zelati ${ }^{\mathrm{k}}$, V. De Falcol ${ }^{1}$ D. de Martino ${ }^{\mathrm{m}}$, T. Di Salvo ${ }^{\mathrm{n}}$, P. Esposito ${ }^{\mathrm{o}, \mathrm{e}}$, C. Ferrigno ${ }^{\mathrm{i}}$, M. Forot ${ }^{\mathrm{p}}$, D. Götz ${ }^{\mathrm{p}}$, C. Gouiffes $^{\mathrm{q}}$, R. Iaria ${ }^{\mathrm{n}}$, P. Laurent ${ }^{\mathrm{p}}$,

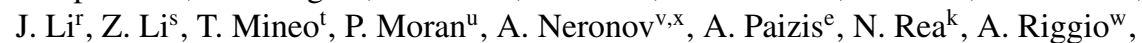

A. Sanna ${ }^{\text {w }}$, V. Savchenko ${ }^{i}$ A. Słowikowska ${ }^{y}$, A. Shearer ${ }^{\mathrm{v}}$, A. Tiengo ${ }^{\text {o,e }}$, D. F. Torres ${ }^{\mathrm{k}, \mathrm{z}, \mathrm{aa}}$

\footnotetext{
${ }^{a}$ INAF-Osservarorio Astronomico di Roma, via Frascati 33, Monte Porzio Catone, I-00078 Italy

${ }^{b}$ International Space Science Institute (ISSI), Hallerstrasse 6, CH-3012 Bern, Switzerland ${ }^{c}$ SRON Netherlands Institute for Space Research, Sorbonnelaan 2, NL-3584 CA Utrecht, the Netherlands

${ }^{d}$ Anton Pannekoek Institute for Astronomy, University of Amsterdam, Science Park 904, NL-1098 XH Amsterdam, the Netherlands

e INAF Istituto di Astrofisica Spaziale e Fisica Cosmica, Via A. Corti 12, I-20133 Milano, Italy

${ }^{f}$ Tuorla Observatory, Department of Physics and Astronomy, 20014, University of Turku, Finland ${ }^{g}$ Space Research Institute of the Russian Academy of Sciences, Profsoyuznaya str. 84/32, 117997, Moscow, Russia

${ }^{h}$ Nordita, KTH Royal Institute of Technology and Stockholm University, Roslagstullsbacken 23, 10691, Stockholm, Sweden

iISDC, Department of Astronomy, University of Geneva, Chemin d'Ecogia 16, 1290, Versoix, Switzerland jINAF Istituto di Astrofisica e Planetologia Spaziali (IAPS), Via del Fosso del Cavaliere 100, I-00133 Rome, Italy

${ }^{k}$ Institute of Space Sciences (ICE, CSIC), Campus UAB, Carrer de Can Magrans, E-08193, Barcelona, Spain

${ }^{l}$ Research Centre for Computational Physics and Data Processing, Faculty of Philosophy E Science, Silesian University in Opava, Bezruovo namesti 13, CZ-746 01 Opava, Czech Republic

${ }^{m}$ INAF Osservatorio Astronomico di Capodimonte, Salita Moiariello 16, I-80131 Napoli, Italy

${ }^{n}$ Università degli Studi di Palermo, Dipartimento di Fisica e Chimica - Emilio Segr, via Archirafi 36, I-90123 Palermo, Italy

${ }^{o}$ Scuola Universitaria Superiore IUSS Pavia, piazza della Vittoria 15, 27100, Pavia, Italy

${ }^{p}$ CEA, IRFU, Service d'Astrophysique, Orme des Merisiers, 91191 Gif-sur-Yvette, France

${ }^{q}$ Laboratoire AIM, UMR 7158 (CEA/Irfu, CNRS/INSU, Universit Paris VII), CEA Saclay, Bt. 709, F-91191 Gif-sur-Yvette Cedex, France

'Deutsches Elektronen Synchrotron DESY, D-15738 Zeuthen, Germany

Department of Physics, Xiangtan University, Xiangtan, 411105, PR China

${ }^{t}$ INAF Istituto di Astrofisica Spaziale e Fisica Cosmica Palermo, Via U. La Malfa 153, I-90146 Palermo, Italy

${ }^{u}$ Centre for Astronomy, School of Physics, National University of Ireland Galway, University Road, Galway, Ireland

${ }^{v}$ Astronomy Department, University of Geneva, Ch. d'Ecogia 16, 1290, Versoix, Switzerland ${ }^{w}$ Dipartimento di Fisica, Università di Cagliari, SP Monserrato-Sestu, Km 0.7, I-09042 Monserrato, Italy ${ }^{x}$ APC, Astroparticule et Cosmologie CNRS/IN2P3, CEA/IRFU, 10 rue Alice Domon et Leonie Duquet, F-75013, Paris, France

${ }^{y}$ Institute of Astronomy, Faculty of Physics, Astronomy and Informatics, Nicolaus Copernicus University in Toruń, Grudziadzka 5, PL-87-100 Toruń, Poland

${ }^{z}$ Institut d'Estudis Espacials de Catalunya (IEEC), E-08034 Barcelona, Spain
}

Email address: alessandro.papitto@inaf.it (A. Papitto) 


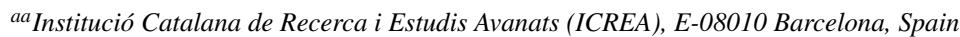

\begin{abstract}
In the last 25 years a new generation of X-ray satellites imparted a significant leap forward in our knowledge of X-ray pulsars. The discovery of accreting and transitional millisecond pulsars proved that disk accretion can spin up a neutron star to a very high rotation speed. The detection of $\mathrm{MeV}-\mathrm{GeV}$ pulsed emission from a few hundreds of rotation-powered pulsars probed particle acceleration in the outer magnetosphere, or even beyond. Also, a population of two dozens of magnetars has emerged. INTE$G R A L$ played a central role to achieve these results by providing instruments with high temporal resolution up to the hard X-ray/soft, gamma-ray band and a large field of view imager with good angular resolution to spot hard X-ray transients. In this article we review the main contributions by INTEGRAL to our understanding of the pulsating hard X-ray sky, such as the discovery and characterization of several accreting and transitional millisecond pulsars, the generation of the first catalog of hard X-ray/soft $\gamma$ ray rotation-powered pulsars, the detection of polarization in the hard X-ray emission from the Crab pulsar, and the discovery of persistent hard X-ray emission from several magnetars.
\end{abstract}

Keywords: accretion disks, magnetars, neutron stars, pulsars, $\mathrm{X}$-rays: binaries, $\mathrm{X}$-rays: bursts

\title{
1. Introduction
}

Since 1967, the pulsar phenomenon has provided the largest share of information we have obtained so far on the properties of neutron stars (henceforth NS) [1, 2, 3]. By now, emission coherently modulated by the NS rotation has been observed at all wave5 lengths, from the radio to the very high energy domains. A few physical mechanisms can produce pulsations observed at X-ray and soft gamma-ray energies covered by $I N$ TEGRAL, namely magnetically channeled accretion of matter transferred from a companion star in a binary system, spreading of the thermonuclear burning front over the NS surface resulting from the ignition of accreted matter (so-called type-I X-ray burst oscillations), pulsed emission powered by the rotation of the strong electro-magnetic field anchored to the NS surface and/or the dissipation of such a field in magnetars. Observing pulsars has been crucial to measure fundamental properties of NSs (such as masses, radii, magnetic fields) and understand their evolution (see, e.g., [4]).

In this chapter we review the main results achieved by the INTEGRAL mission [5] on accreting and transitional millisecond pulsars, rotation-powered pulsars and magnetars. In this regard, the hard X-ray $(20 \mathrm{keV}-1 \mathrm{MeV})$ imager IBIS/ISGRI [6, 7] played a major role, as it combined a large field of view $\left(29^{\circ} \times 29^{\circ}\right.$ with a fully coded field of $\left.8^{\circ} \times 8^{\circ}\right)$, fine angular resolution $\left(12^{\prime}\right.$ full-width half-maximum) and high temporal resolution $(60 \mu \mathrm{s},[8])$. The X-ray (3-35 keV) monitor JEM-X [9] complemented these 
properties providing a better angular resolution of $3^{\prime}$ and sensitivity to softer energies, although with a smaller field of view (the fully illuminated part is $\sim 4.8^{\circ} \times 4.8^{\circ}$-wide).

The structure of the article is the following. In Sec. 2 we review the INTEGRAL contribution to the study of accreting millisecond pulsars, Gyr-old and relatively weakly magnetized $\left(\simeq 10^{8}-10^{9} \mathrm{G}\right)$ NSs that were spun-up to their current fast spin period $(P \simeq 1-10 \mathrm{~ms})$ by the accretion of matter transferred from a low mass companion star $\left(\leq M_{\odot}\right)$. These sources also show bursts of soft X-rays caused by the thermonuclear burning of the material accreted on the surface and the INTEGRAL results are summarized in Sec. 2.5. We refer the reader to the article by Sazonov et al. for the results obtained for slower and/or non-pulsating NSs in low-mass X-ray binaries, and to the 30 article by Kretschmar et al. for the case of X-ray pulsars in high-mass X-ray binaries, both in this volume. Sec. 3 describes the role played by INTEGRAL in discovering and characterizing transitional millisecond pulsars, a small sample of sources that are able to alternate between phases of emission as a low-mass X-ray binary and regimes characterized by radio pulsar emission. Studies of pulsars powered by the rotation of

35 their magnetic field, including both slower $(P \sim 0.1-10 \mathrm{~s})$ and strongly magnetized $\left(B \sim 10^{11}-10^{13} \mathrm{G}\right)$ classical pulsars and recycled millisecond pulsars $(P \sim 1-10 \mathrm{~ms}$; $\left.B \sim 10^{8} \mathrm{G}\right)$ are presented in Sec. 4. Finally, INTEGRAL observations of NSs powered by the dissipation of their intense $\left(B \sim 10^{13}-10^{14} \mathrm{G}\right)$ magnetic field (so-called magnetars) are summarized in Sec. 5.

\section{2. Accreting millisecond pulsars}

Accreting millisecond pulsars (AMSPs in the following) are NSs that transiently accrete the plasma captured from a low-mass companion star $\left(M_{2} \leq M_{\odot}\right)$ via Rochelobe overflow ([10]; see $[11,12]$ for reviews). Their magnetic field $\left(B_{p} \simeq 10^{8}-10^{9} \mathrm{G}\right)$ is strong enough to truncate the disk in-flow before the plasma reaches the surface of

45 the NS. The in-falling matter is then channeled by the magnetic field of the NS to the magnetic polar regions of the NS surface. As long as the magnetic and spin axes are misaligned and the emission beam crosses the line of sight, this produces coherent pulsations mainly observed in the X-ray domain.

The spin periods of AMSPs range between 1.6 and $~ 10 \mathrm{~ms}$. According to the 50 recycling evolutionary model $[13,14,15]$, such an extremely quick rotation is achieved during a prolonged $(\approx 0.1-1 \mathrm{Gyr}) \mathrm{X}$-ray bright phase of accretion of matter lost by a low-mass companion star. The $\sim 300$ millisecond radio pulsars known to date are then assumed to be the descendants of low mass X-ray binaries (LMXBs in the following). In these systems, a radio pulsar turns on as soon as the pressure of the 5 pulsar wind inhibits the in-fall of matter lost by the companion and accretion ceases (see also Sec. 3).

So far, AMSPs have been found in binaries hosting either a main sequence star $\left(M_{2} \sim 0.1-0.5 \mathrm{M}_{\odot}, P_{\text {orb }} \approx\right.$ a few hours $)$, a brown dwarf $\left(M_{2} \approx 0.05 \mathrm{M}_{\odot}, P_{\text {orb }} \simeq 1-2\right.$ hr) or a white dwarf $\left(M_{2} \approx 0.01 \mathrm{M}_{\odot}, P_{\text {orb }} \simeq 40 \mathrm{~min}\right)$ were found. These values are

60 similar to those of binary millisecond radio pulsars whose signal is irregularly eclipsed by matter ejected by the pulsar wind and engulfing the binary system. These eclipsing radio pulsars are dubbed either black widow $\left(M_{2} \approx 0.05 \mathrm{M}_{\odot},[16,17]\right)$ or redback 


\begin{tabular}{||lcccl||}
\hline Source & $P_{\text {spin }}(\mathrm{ms})$ & $P_{\text {orb }}(\mathrm{hr})$ & Outburst year & References \\
\hline INTEGRAL sources & \multicolumn{4}{|c}{} \\
\hline \hline IGR J00291+5934 & 1.7 & 2.46 & $2004,{ }^{\prime} 15$ & {$[28,29,30]$} \\
IGR J17511-3057 & 4.1 & 3.47 & 2009 & {$[31]$} \\
IGR J17498-2921 & 2.5 & 3.84 & 2011 & {$[32]$} \\
IGR J17480-2446 & 90 & 21.3 & 2011 & {$[33,34,35,36]$} \\
IGR J18245-2452 & 3.9 & 11.0 & 2013 & {$[37,38,39]$} \\
IGR J17062-6143 & 6.1 & $>0.3$ & 2008 & {$[40]$} \\
IGR J16597-3704 & 9.5 & 0.77 & 2017 & {$[41]$} \\
IGR J17379-3747 & 2.1 & 1.88 & 2018 & {$[42,43]$} \\
IGR J17591-2342 & 1.9 & 8.80 & 2018 & {$[44]$} \\
\hline Other s0urces & & & & \\
SAX J1808.4-3658 & 2.5 & 2.01 & $2008, ' 15,19$ & {$[45,46,47]$} \\
XTE J1751-305 & 2.3 & 0.71 & $2005, ' 07, ' 09$ & {$[48,49,50]$} \\
XTE J1807-294 & 5.3 & 0.67 & 2003 & {$[51]$} \\
HETE 1900.1-2455 & 2.7 & 1.39 & 2005 & {$[52]$} \\
SAX J1748.9-2021 & 2.3 & 8.77 & $2015, ' 17$ & {$[53,54,55]$} \\
Swift J1749.4-2807 & 1.9 & 8.82 & 2010 & {$[56,57,58]$} \\
MAXI J0911-655 & 2.9 & 0.74 & 2016 & {$[59,60,61]$} \\
\hline
\end{tabular}

Table 1: AMSPs observed by INTEGRAL.

pulsars $\left(M_{2} \simeq 1 \mathrm{M}_{\odot},[18,19]\right)$ depending on the mass of the companion star, and are generally considered to share a close evolutionary link with AMSPs [20, 21, 22].

Discovering AMSPs and measuring their spin evolution is crucial to understand what is the maximum spin that can be reached by a NS through accretion. In turn, this indirectly probes whether continuous gravitational-wave spin-down torques are required to limit the accretion driven spin up to the minimum observed period of a millisecond pulsar, $\approx 1.5 \mathrm{~ms}[23,24,25]$. The X-ray pulsed emission of AMSPs is

70 emitted close to the surface of a rapidly rotating object that attains a speed of up to $\approx 15 \%$ of the speed of light at the equator. General and special relativity effects shape the energy and trajectory of X-ray photons in a way that can be disentangled through $\mathrm{X}$-ray pulse profile fitting. This makes AMSPs among the best candidates to measure simultaneously the mass and radius of a NS and draw constraints on its equation of 75 state ([26]; see also [27] for a recent review).

\subsection{Discovery and follow-up of AMSPs with INTEGRAL}

The two dozens of AMSPs discovered so far are all X-ray transients. They undergo a few weeks-long X-ray outbursts and spend most of the time in quiescence, although episodes of X-ray activity lasting up to a few years have also been observed.

80 Recurrence times range from a few months to more than 15 years, for sources which have been observed only once, so far. During outbursts, the mass accretion rate rarely exceeds a few per cent of the Eddington rate $\left(L_{X} \approx 10^{36}-10^{37} \mathrm{erg} \mathrm{s}^{-1}\right)$, whereas in quiescence the luminosity is much lower $\left(L_{X} \lesssim 10^{32} \mathrm{erg} \mathrm{s}^{-1}\right)$. The discovery of new 
systems of this class requires instruments with a large field of view and a good sensi85 tivity.

The instruments on-board INTEGRAL perfectly satisfy these requirements and managed to discover the X-ray outbursts of eight sources that were later identified as AMSPs (out of a total of 22; see Table 1, [62] and references therein), and to study their X-ray emission from the onset of the outbursts nearly down to the return in qui90 escence. Since the launch of INTEGRAL the large field of view of the IBIS/ISGRI imager has been exploited to monitor regularly the Galactic bulge $[63,64]$, where most of the transient LMXBs are expected. The highly eccentric long orbit and the special pointing strategy adopted by INTEGRAL allowed IBIS/ISGRI to accumulate several thousands of kiloseconds of observations in each monitored region, with a few-hours

95 long uninterrupted coverage, and achieve a hard $(20-200 \mathrm{keV}) \mathrm{X}$-ray flux sensitivity as low as a few $\times 10^{-11} \mathrm{erg} \mathrm{cm}^{-2} \mathrm{~s}^{-1}$ even in crowded regions. For a distance between $5-8 \mathrm{kpc}$, this limiting sensitivity corresponds to a luminosity of $\approx 10^{35} \mathrm{erg} \mathrm{s}^{-1}$, which is usually reached by AMSPs already during the earliest stages of their outbursts, and attained again towards the end of the outbursts before the switch back to quiescence. IBIS/ISGRI observations were also often complemented by data in the soft band covered by JEM-X at similar sensitivity.

These features, complemented by the good angular resolution of the two instruments, proved crucial to discover AMSPs and disentangle their emission from foreground sources (see Fig. 1). These imaging capabilities were particularly important to identify IGR J00291 +5934, which is located only $\sim 18^{\prime}$ from the close-by persistent intermediate polar V609 Cas [29], and IGR J17511-3057, which is located only 20' away from the other AMSP XTE J1751-305 and whose discovery outburst was partly contaminated by a faint activity episode of the latter source ([31], see Fig. 2. The good angular resolution of IBIS/ISGRI and JEM-X has also proven fundamental to clearly distinguish the type-I X-ray bursts emitted by the AMSP IGR J17498-2921 from those going off in the nearby bursters SLX 1744-300/299 and 1A 1742-294, located at $0.9^{\circ}$ and $0.86^{\circ}$, respectively, from the AMSP [32]. In 2010 INTEGRAL also discovered the LMXB IGR J17480-2446 in the globular cluster Terzan 5 [33, 34]. The source was subsequently identified as a 90 ms pulsar [35] orbiting a low-mass companion star in a $21 \mathrm{hr}$ orbit ([36], see Sec. 2.5 for details).

INTEGRAL observations have also been extensively carried out to follow up AMSPs first detected by other facilities and which underwent one or multiple outbursts since the beginning of the mission science operations in 2002. In a few cases (e.g. XTE J1751-305, SAX J1748.9-2021, SAX J1808.4-3658), the INTEGRAL seasonal pointings toward the Galactic bulge detected the onset of some of the outbursts of AMSPs already discovered. The LMXB nature of Swift J1749.4-2807 was first established during its 2010 outburst announced by INTEGRAL [56, 57]; the source was later indentified as a $1.9 \mathrm{~ms}$ AMSP which showed 1.7 ks-long X-ray eclipses [see, e.g., 58, 65], making it the first and only eclipsing AMSP discovered so far, with important consequences for the determination of the NS mass and radius [66]. The first outburst of MAXI J0911-655, was also observed by INTEGRAL [59, 60, 61], which also provided a long term monitoring of the source reporting on the discovery of significant hard X-ray emission more than 450 days after the onset of the event [67]. 


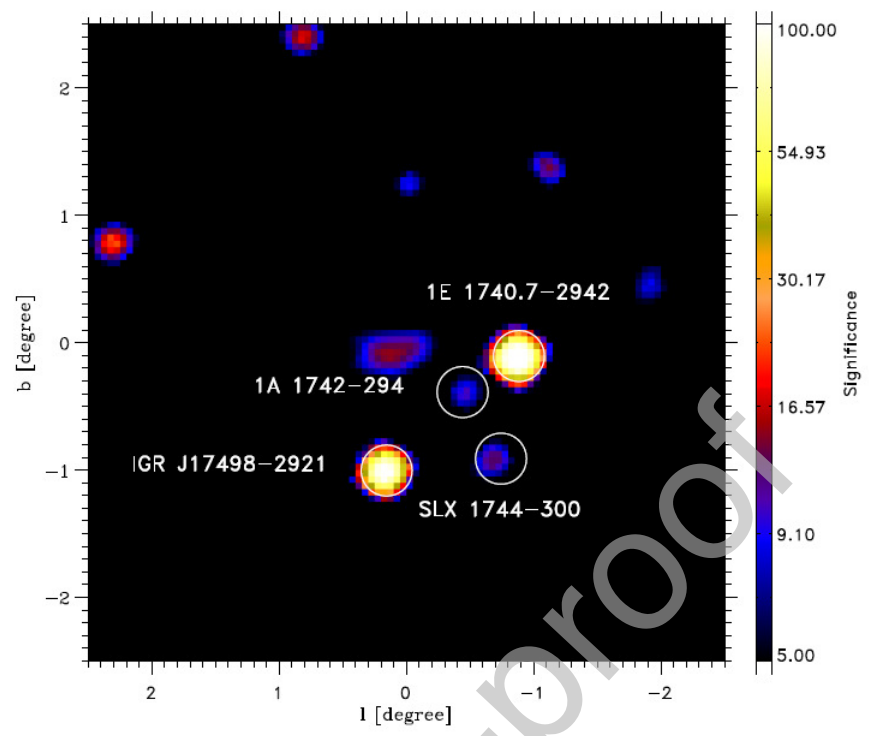

Figure 1: IBIS/ISGRI 20-100 keV sky image of the field around IGR J17498-2921 obtained during observations performed at the time of the source discovery in 2011, giving an effective exposure of $210 \mathrm{ks}$ (image taken from [32]).

\subsection{The $X$-ray light curves} which have been important to extract light-curves of AMSP outbursts and identify the physical mechanisms driving the outburst onset and decay. The light curves observed were generally characterized by a fast rise (a few days) and an exponential decay (up to several weeks) which terminated with a break followed by a linear decay extending This behaviour has been commonly interpreted in terms of the disk instability model, in which the irradiation of the accretion disk by the central X-ray source plays a key role in the shaping of the light curve profile [68]. Modelling of the light curves observed by the Rossi X-ray Timing Explorer (RXTE, [69]) showed that the timescale of the decay and its luminosity at a characteristic time are linked to the outer radius of the accretion disk [70]. The break observed during the X-ray flux decay at the end of the outburst is thought to be associated with the lowest X-ray luminosity at which the outer disk region can be kept in a hot high-viscosity state by the centrally illuminating source. When the outer disk region enters the cool low-viscosity state, the mass accretion rate onto the compact object is effectively cut-off and the source starts its return to quiescence. The application of this model to the INTEGRAL data gave compatible results [29, 31, 32, 58].

So far, only IGR J00291+5934 has shown a double-peaked outburst [71, 72], while a few other AMSPs have undergone "re-flares" toward the later stages of the return to 


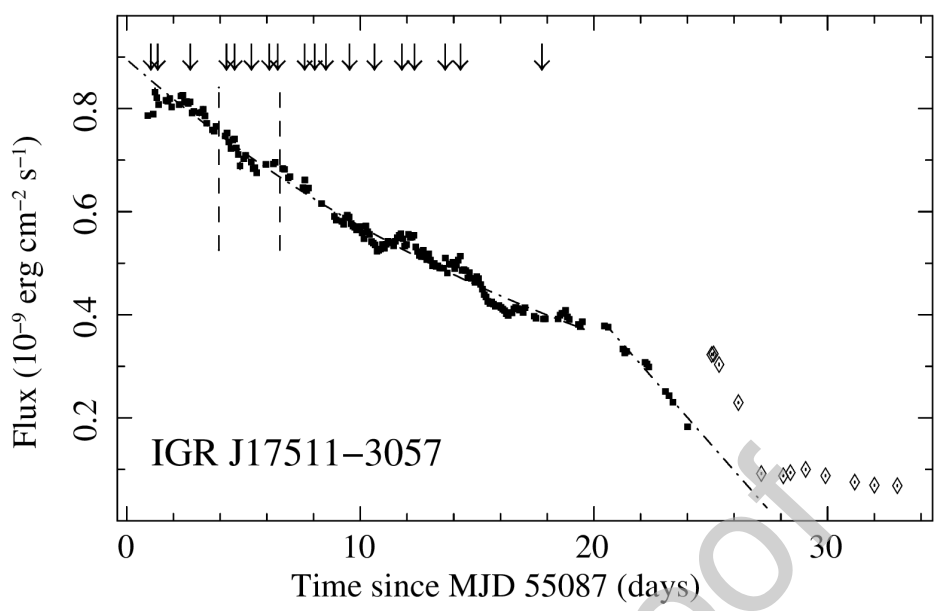

Figure 2: The light curve of the outburst of IGR J17511-3057 observed in 2009 [31]. The typical exponential decay profile is clearly visible, with a break occurring about 20 days after the onset of the event. The light curve is obtained from the RXTE/PCA $(2-20 \mathrm{keV})$ data, with the vertical dashed lines indicating the interval of the INTEGRAL observations. The arrows mark the times of the detected X-ray bursts. The diamonds refer to the observations in which both IGR J17511-3057 and XTE J1751-305 were active and the instruments on-board RXTE were unable to separate the contribution of the two sources.

quiescence. The mechanism(s) driving these re-brightening episodes is still a matter of debate [see, e.g., 73, 74, and references therein]. The typical luminosity at which the re-flares occur is close to the detection limit for both IBIS/ISGRI and JEM-X and thus these events are hardly observable by INTEGRAL.

\subsection{The hard X-ray spectra of AMSPs}

The spectra of AMSPs in outburst measured by INTEGRAL are typically hard and dominated by a power law $d F / d E \propto E^{-\Gamma}$, with photon index $\Gamma \sim 2$, extending up to 100 $\mathrm{keV}$ or beyond [75]. Most likely, these hard X-ray photons originate from the accretion columns above the polar caps, where electrons energized by the shock between the infalling plasma and the NS surface up-scatter the surface soft photons to higher energies $[76,77]$. In most cases, the combined IBIS/ISGRI+JEM-X spectra could be well fit with a thermal Comptonization model comprs [78] in the slab geometry assumed for the accretion columns $[29,31,32,51,52,58]$. The main model parameters are the Thomson optical depth $\tau_{\mathrm{T}} \sim 1-3$ across the slab, the electron temperature $k T_{\mathrm{e}} \sim 25-50$ $\mathrm{keV}$, the temperature $k T_{\mathrm{bb}} \sim 0.3-1.0 \mathrm{keV}$ of the soft-seed blackbody photons (assumed to be injected from the bottom of the slab), and the emission area $A_{\mathrm{bb}} \sim 20 \mathrm{~km}^{2}$. Fig. 3 shows a typical broad-band AMSP spectrum observed during the decay of an outburst, fitted with a compPs model. The spectrum observed during an outburst of the transitional millisecond pulsars IGR J18245-2452 is instead significantly harder, with a photon index $\Gamma \sim 1.3$ and seed photons coming from a larger region and characterized by a lower temperature ([39], see Sec. 3.1). INTEGRAL observations of Aql X-1, which has been detected as an AMSP only for a couple of minutes [79], also caught the source in a state characterized by a very hard spectrum extending up to $150 \mathrm{keV}$ [80]. 


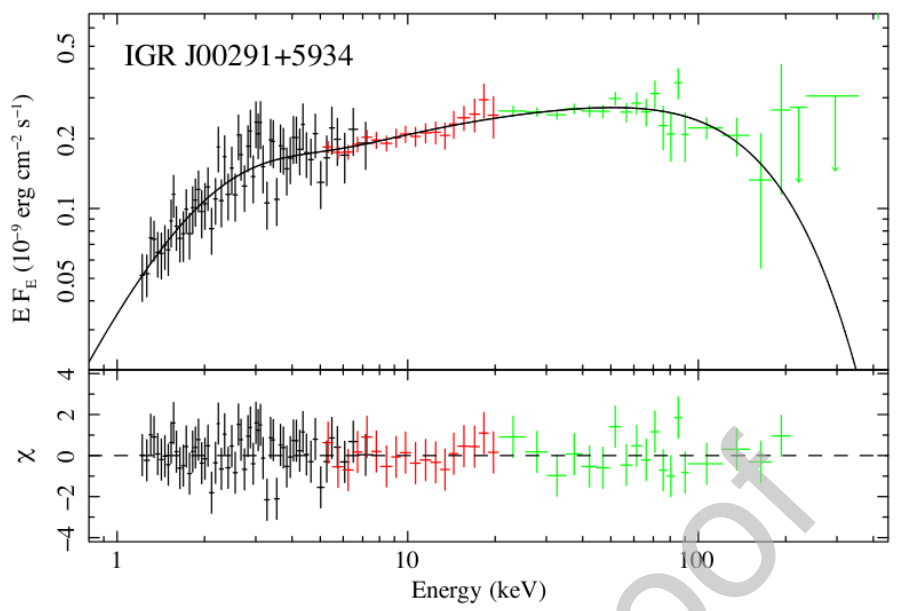

Figure 3: The unfolded broad-band spectrum of the AMSP IGR J00291+5934 measured by JEM-X (red points) and IBIS/ISGRI (green points) during an outburst exhibited in 2015. Swift/XRT data were also used for the analysis and are shown as black points. The best fit is obtained with the comPPs model (solid black line), resulting in a plasma temperature of $k T \sim 50 \mathrm{keV}$. The bottom panel shows the residuals from the best fit [figure from 30].

The spectra of several INTEGRAL-detected AMSPs have been also observed by using combined INTEGRAL and (quasi-)simultaneous XMM-Newton [81] and NuSTAR [82] observations. Although they have been clearly found to be dominated by hard power-law components, typically ascribed to the Comptonization of seed thermal photons in agreement with previous INTEGRAL results, the extension into the soft X-ray regime frequently revealed the presence of a additional thermal components originating from hot spots on the NS surface or from the inner disk boundary. In addition, a broad iron line produced by the reflection of the X-ray photons onto the inner regions of the accretion disk surrounding the compact object was sometimes also observed $[83,84,85,44,86]$. In at least one case, high spectral resolution observations carried out with the gratings on-board Chandra were able also to detect outflows from the outer regions of the accretion disk [87], and to provide hints of an expanding hot corona with high outflow velocities also in the case of IGR J00291+0034 [88].

Little spectral variability, if any, has been generally observed during the course of an outburst. SAX J1748.9-2021 in the globular cluster NGC 6440 clearly made an exception. It has been observed to switch between hard and soft spectra [89] such as occurs in the so-called "Atoll" sources [90]. The combined extensive monitoring performed with IBIS/ISGRI and JEM-X during the source outburst in 2015 was able to efficiently catch one of these spectral state changes ([54]; see Fig. 4); a dramatic transition from a hard to a soft state state occurred over roughly half a day, about ten days after the onset of the outburst. In that outburst the source reached a peak luminosity of about $5 \times 10^{37} \mathrm{erg} \mathrm{s}^{-1}$, a value higher than usually observed from AMSPs.

After the state change, the spectrum observed with XMM-Newton appeared to be very soft with a Comptonization electron temperature around $2 \mathrm{keV}$ [91]. The (quasi) si- 


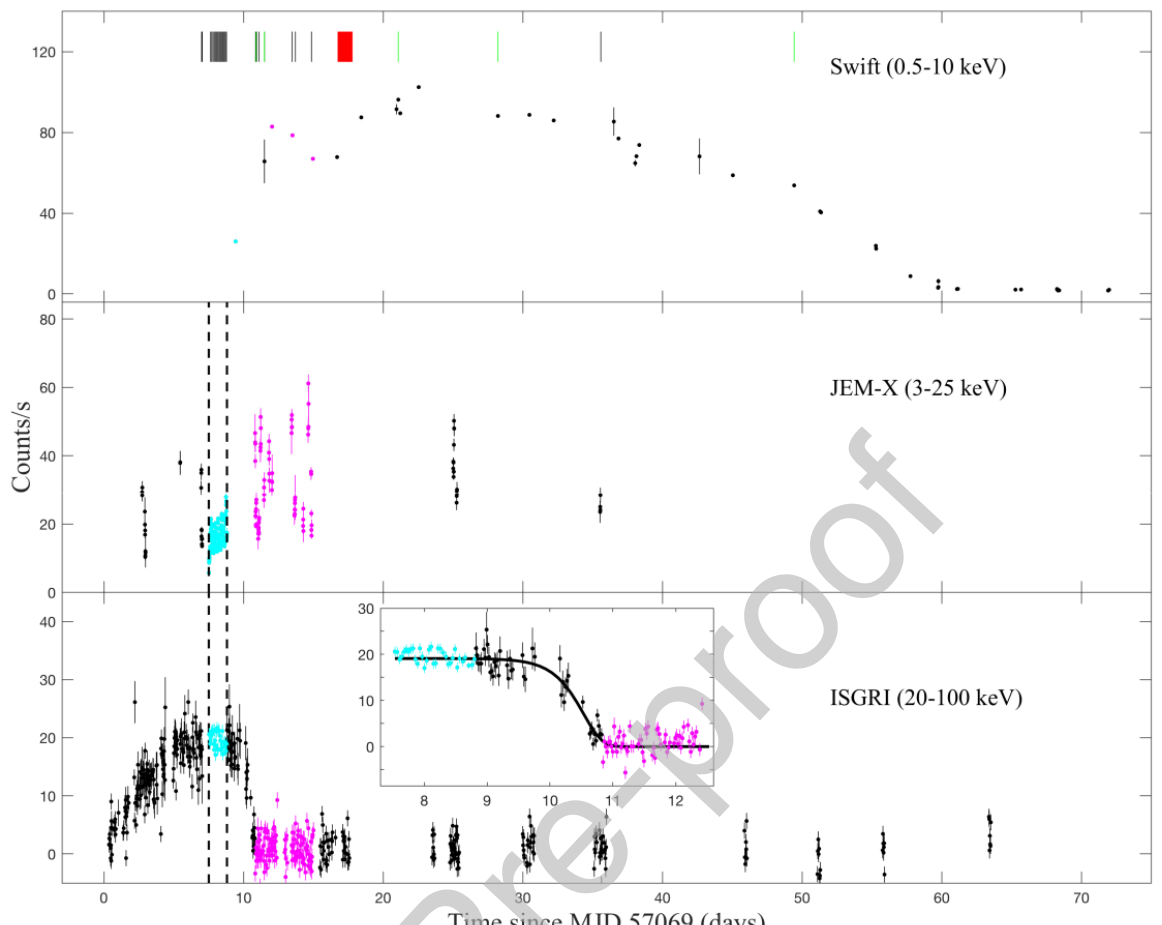

Figure 4: The light curve from the 2015 outburst of the AMSP SAX J1748.9-2021. The top panel shows data collected with Swift/XRT, while the two panels below report the JEM-X and IBIS/ISGRI data. The spectral transition from hard (light blue) to soft (magneta) state is clearly visible in the INTEGRAL data (see the inset of the bottom panel). The figure also shows the time of a dedicated $100 \mathrm{ks}-$ long INTEGRAL ToO observation (marked with vertical dashed lines) and all the thermonuclear bursts recorded during the outburst with INTEGRAL, Swift, and XMM-Newton (black, green, and red vertical lines, respectively). Figure taken from [54].

multaneous spectrum observed with INTEGRAL also revealed the presence of a hard power-law component with a photon index of 2.3. Similar hard tails are often observed in Z-sources (see e.g. the INTEGRAL spectrum of Sco X-1, [92, 93]) and atoll sources in the soft state (see e.g. the IBIS/ISGRI spectrum of GX 13+1 [94], and the XMM-Newton/INTEGRAL spectrum of GX 3+1 [95]) and are interpreted in terms of Comptonization of photons off electrons with a non-thermal distribution of velocity. This non-thermal component may be related to high energy electrons injected in the Comptonization region by a (failed?) jet or powered by magnetic reconnections close to the accretion disk. During the outburst occurred in October 2017, SAX J1748.92021 showed instead a standard outburst X-ray luminosity of $3 \times 10^{36} \mathrm{erg} \mathrm{s}^{-1}$ and a more common hard spectrum (photon index $\Gamma \sim 1.6-1.7$, and electron temperature of $20 \mathrm{keV}$, [96]). This demonstrates the importance of a high-energy monitor such as INTEGRAL for addressing the spectral state of these sources and individuate peculiar ones. 


\subsection{The hard $X$-ray pulse profiles}

AMSPs are relatively faint $X$-ray transients and the amplitude of their pulsations is $\lesssim 10$ per cent, [11]. For this reason, usually X-ray pulsations could not be discovered independently by INTEGRAL without a previous detection by a large area focusing or collimated instruments, as those on-board RXTE, XMM-Newton, and more recently NUSTAR and The Neutron Star Interior Composition Explorer Mission (NICER, [97]). Only during the 2015 outburst of IGR J00291+5934, did INTEGRAL data statistics reach a sufficient level to measure the pulsar ephemeris directly from the IBIS/ISGRI event files [98], obtaining parameters compatible with those derived by RXTE. Once the spin and orbital parameters of an AMSP were provided, the INTEGRAL data could be folded to increase the signal-to-noise ratio and carry out a pulse timing analysis in a very broad energy range, covering up to a few hundreds of $\mathrm{keV}$. In this way, IBIS/ISGRI detected X-ray pulsations up to $150 \mathrm{keV}$ from IGR J00291+5934 [29], IGR J17511-3057 [31], IGR J17498-2921 [32], and IGR J18245-2452 [39], while an accurate analysis of the data has not been published yet for recently discovered IGR AMSPs, such as IGR J16597-3704, IGR J17379-3747, IGR J17591-2342 and IGR J17062-6143 (Kuiper et al. in prep.). The measurements obtained by IBIS/ISGRI permitted to perform an analysis of the pulsed fractions in a hard ( $>20 \mathrm{keV}) \mathrm{X}$-ray domain previously poorly covered by other instruments. Different trends were observed from source to source. IGR J17511-3057, IGR J17498-2921 and IGR J18245-2452 showed a constant, or slightly decreasing pulsed fraction above $10 \mathrm{keV}$, compatible with the results obtained by RXTE for SAX J1808.4-3658 and XTE J1751-305 (see left panels of Fig. 5 labelled as (b) and (c); see [99] and references therein). A decrease of the pulsed fraction at high energies has been explained in the context of the two pulsed components assumed to originate from the hot-spot on the NS surface and from the accretion column above the poles ([76, 77], see Sec. 2.3$)$. The soft blackbody radiation from the hot-spots is more beamed along the axis than the hard photons produced by Compton up-scattering in the accretion columns, naturally accounting for the lower pulsed fraction observed at higher energies [26]. On the other hand, a clear increase of 240 the pulsed fraction with energy above $\sim 50 \mathrm{keV}$ was observed from IGR J00291+5934 (see left panel labelled as (a) in Fig. 5). Embedding of the accretion columns in a Compton cloud was proposed to explain this [99]. the electron cross section and the resulting Compton optical depth decrease in fact at high energies, allowing a larger fraction of the pulsed harder X-rays to reach the observer un-scattered. Note that a trend of increasing pulsed fraction with energy, albeit already at softer X-rays, was observed by other missions (e.g., RXTE, XMM-Newton and NuSTAR) also from SAX J1748.9-2021 [89, 100], Swift J1756.9-2508 [101, 102] and, to a lesser extent, Swift J1749.4-2807 [58].

INTEGRAL crucially contributed also to reveal the complex dependence of time lags of pulsed photons with energy (see right panel of Fig. 5, taken from [99]). The measured soft lags mean that the low-energy pulses are delayed relative to pulses at higher energies. Below $10 \mathrm{keV}$, soft photons generally lag hard photons, although the opposite behavior was observed during an outburst of the transitional millisecond pulsar IGR J18245-2452 (see Sec. 3.1). Observed soft phase/time lags were explained either in terms of down-scattering of hard photons emitted in the accretion columns by the colder surrounding plasma $[99,103,104]$ or by the broader emission pattern 

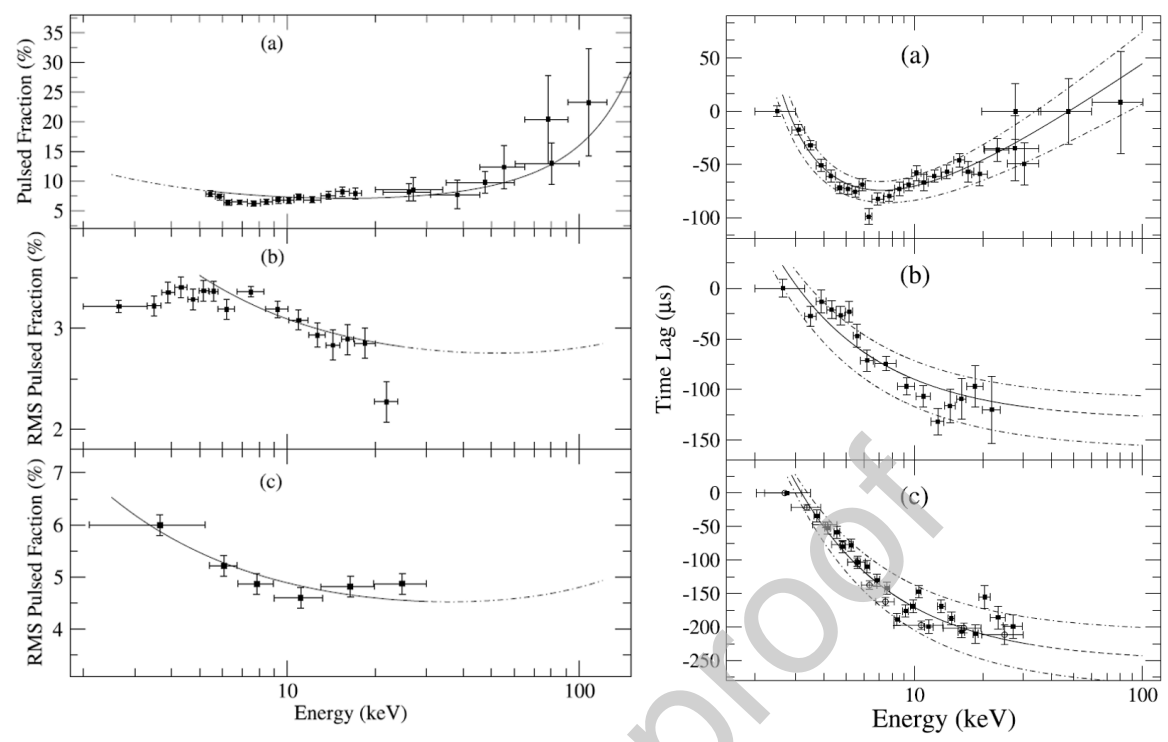

Figure 5: The pulsed fraction (panel (a), left) and the time lags of the hard pulse (panel (a), right) measured during the 2005 outburst of the AMSP IGR J00291+5934 using data from the RXTE/PCA, RXTE/HEXTE, INTEGRAL/JEM-X, and INTEGRAL IBIS/ISGRI. Panel (b) and (c) show the same quantities for SAX J1808.4-3658 and XTE J1751-305, as measured by RXTE. Figures adapted from [99].

of hard photons Comptonized in the accretion columns with respect to soft photons emitted from the NS hot spots, which make harder photons to be seen before the softer ones as the NS rotates $[26,77,105]$. Above an energy of $6 \mathrm{keV}$, the dependence of the lags observed from IGR J00291+5934 reverses, and the harder photons $(\sim 100 \mathrm{keV})$ arrive later than the softer $(\sim 10-20 \mathrm{keV})$ ones. In the Comptonization scenario this is explained by the fact that higher energy photons were up-scattered more times and took longer to reach the observer [see, e.g., 32, 99, 31, and references therein].

\subsection{Thermonuclear type-I X-ray bursts}

The INTEGRAL observing strategy generally implies a few-day long observations toward a pre-defined region of the sky. The INTEGRAL monitoring programs aimed at the Galactic bulge, as well as dedicated observational campaigns devoted to specific sources, often offered nearly uninterrupted light curves of all sources in the field and covering a significantly larger fraction of the outbursts of AMSPs with respect to other telescopes. These light curves were particularly useful to search for thermonuclear type-I X-ray bursts and accurately constrain their recurrence time as a function of the mass accretion rate $(\dot{m})$ inferred from the observed non-burst X-ray flux [106]. Fig. 6 shows an example of a particularly intense burst observed by INTEGRAL from the AMSP HETE J1900.1-2455 [52], during which the double peaked profile in the IBIS/ISGRI data proved that a photospheric radius expansion took place [see, e.g., 107, for a recent review]. On the other hand, the light curves of most of the thermonuclear bursts shown by the AMSPs observed by INTEGRAL were characterized 


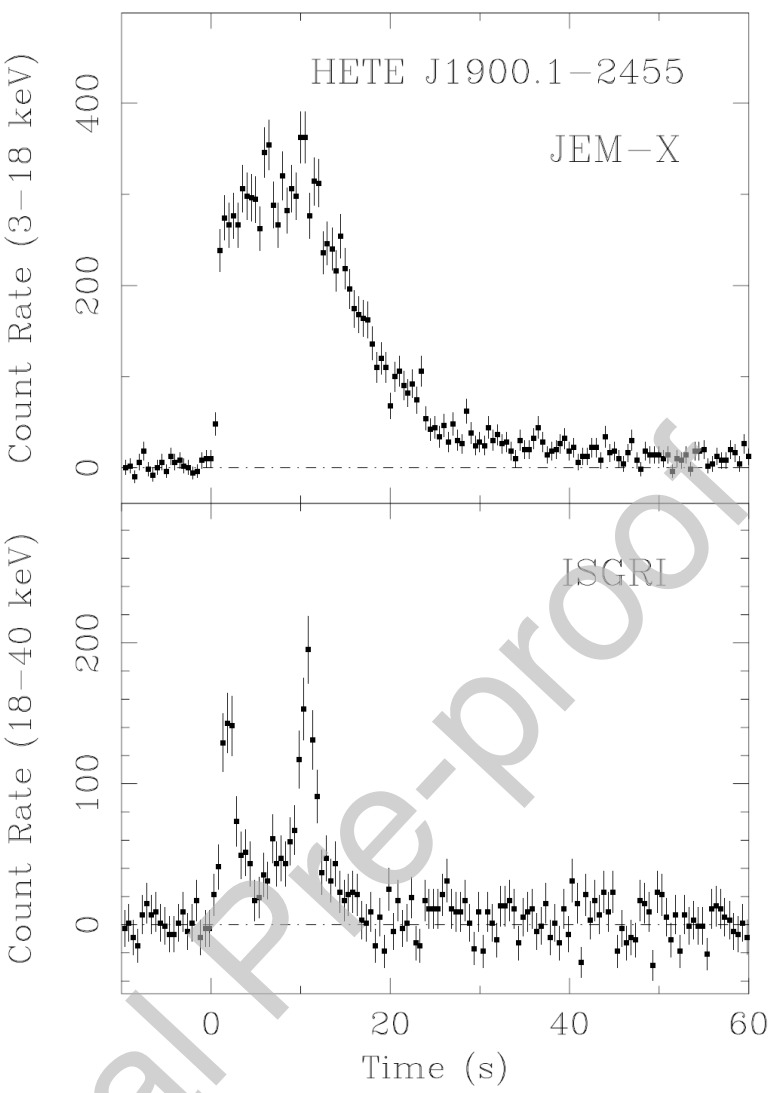

Figure 6: An intense type-I X-ray burst observed by JEM-X and IBIS/ISGRI from the AMSP HETE J1900.1-2455 during its outburst in 2005. The upper panel shows the JEM-X light curve (3-18 keV), while the lower panel shows the IBIS/ISGRI light curve $(18-40 \mathrm{keV})$. The double peaked IBIS/ISGRI light curves clearly show the presence of a photospheric radius expansion [figure from 52].

by a fast rise and an exponential decay lasting a few tens of seconds. Such short burst profiles indicate that the ignition most likely occured in presence of hydrogenpoor material, suggesting that either the accreted material is hydrogen-deficient or that the CNO abundances in AMSPs was slightly higher than the solar value [see, e.g., $30,31,32,39,52,54,58]$. In the case of IGR J17511-3057, this suggestion could be strengthened by the fact that the variation of the burst recurrence time as a function of $\dot{m}$ (see Fig. 7) was found to be much shorter than that predicted in case of helium-ignition models [31].

Among the many thermonuclear bursts observed by INTEGRAL from the more than a hundred of bursting sources known [see, e.g., 108, for a recent catalogue of the bursts observed by JEM-X], it is worth mentioning here the peculiar case of IGR J174802446. This source is located in the Globular Cluster Terzan 5 and underwent so far a 


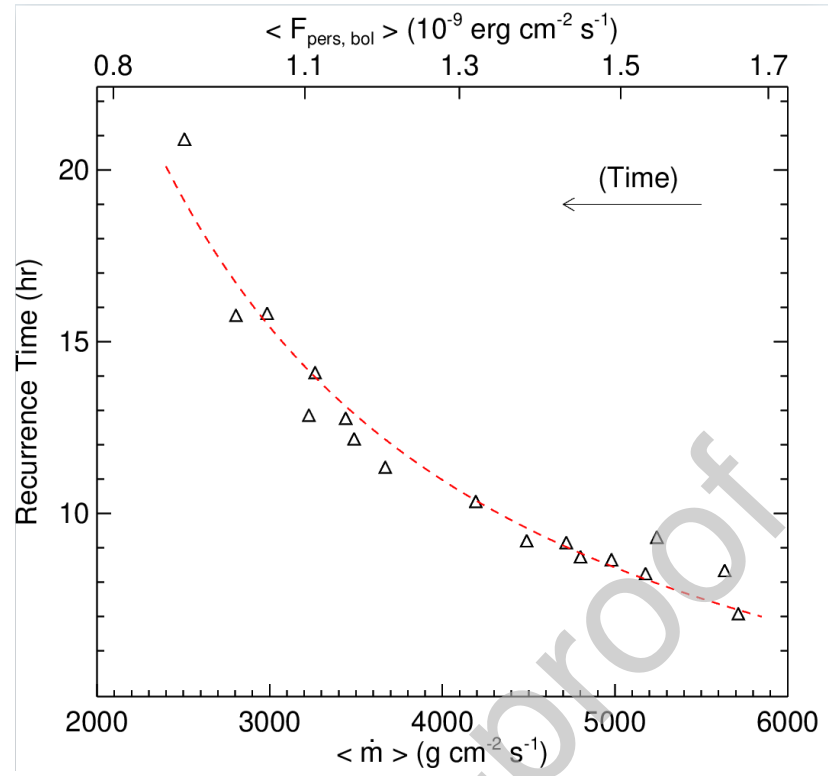

Figure 7: An example of the study of the burst recurrence time in the AMSP IGR J17511-3057 during its 2010 outburst. Triangles in the figure represent the observed burst recurrence times shown as a function of the mass accretion rate per unit area. By using a fit to the data with a power-law model, the recurrence time is found to increase with time roughly as $\left\langle F_{\text {pers,bol }}\right\rangle^{-1.1}$ [figure from 31].

single bright detectable outburst in 2010 [33] which displayed a number of remarkable unique features. First of all, pulsations were clearly detected at a spin period of about $91 \mathrm{~ms}$, making this a unique source linking AMSPs and slower spinning pulsars in LMXBs [35, 36, 109, 110]. Evolutionary calculations suggested that this binary system might have formed through a close encounter between the NS and its companion within the globular cluster, and that the compact object is only a mildly recycled pulsar as accretion did not begin earlier than a few $10^{7}$ yr ago [111]. Furthermore, the source showed several hundreds of thermonuclear bursts along the outburst. The recurrence time markedly decreased during the rising part of the outburst ([112, 113]; see Fig. 8). Close to the outburst peak the frequency of the bursts became so high and their peak flux so close to the level of the persistent emission that they could no longer be identified by visual inspection in the source light curve $([112,114]$, see Fig. 8), but rather emerged as $\mathrm{mHz}$ quasi periodic oscillations in the Fourier power density spectrum [115]. A careful analysis of the spectral softening of the emission during the burst decay proved unequivocally that they had all a thermonuclear origin $[112,116,117]$. Alhough a decrease of the recurrence time with increasing mass accretion rate had been theoretically anticipated [118], this was the first time that it was observed in an LMXB in such great detail. This made IGR J17480-2446 a unique laboratory to test thermonuclear burning models. All the thermonuclear bursts also displayed burst oscillations at a frequency within a few per cent of the spin rotation of the NS, proving for the first time that millisecond rotational velocities are not required to produce these kind of tim- 


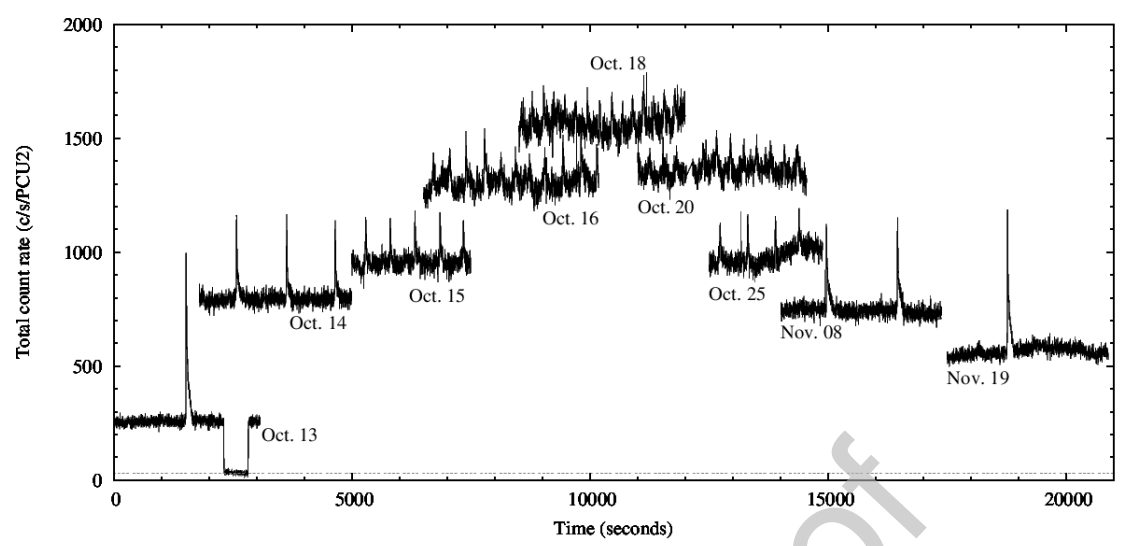

Figure 8: The evolution of the thermonuclear burst frequency in IGR J17480-2446 during the course of its 2010 outburst. Data were obtained from RXTE and the x-axis shows the recurrence time at different phases of the outburst, which evolution of the persistent emission is represented in the y-axis in units of count-rate recorded by the PCU2 on-board RXTE Times have been shifted arbitrarily for display purposes [figure from $113]$.

ing features and ruling out models based on this assumption [119]. IGR J17480-2446 also displayed a clear evidence of a fast-moving disk wind with ejection velocity up to $\sim 3000 \mathrm{~km} \mathrm{~s}^{-1}$, a rare feature in NSs hosted in LMXB and much more common in systems harboring accreting BHs [120]. In addition, the source endured an extremely high level of crustal heating during the outburst, which did not seem to have cooled completely even 5.5 years since the end of the outburst $[121,122,123]$.

Peculiar burst properties have also been observed from SAX J1748.9-2021. The burst recurrence time drastically decreased from $\approx 2$ to $\approx 1 \mathrm{hr}$ as the source underwent an abrupt hard-to-soft state spectral transition ([54], see Fig. 4). The relation between the burst recurrence time and the mass accretion rate indicated that in both states the bursts were consistent with being produced by a mixture of $\mathrm{H}$ and $\mathrm{He}$.

INTEGRAL observations of thermonuclear type-I X-ray bursts from LMXBs also played an important role in the study of the properties of the X-ray coronae from the effect of the burst emission on the surrounding accretion flow (see [124] and references therein). Stacking of 123 bursts observed by INTEGRAL from the persistent LMXB $4 \mathrm{U} 1728-34$ revealed a deficit of hard $40-80 \mathrm{keV}$ photons compared to the persistent emission due to the enhanced corona cooling caused by the soft burst photons [125]; such a deficit had not been detected before by RXTE [126], possibly due to the different response and background contamination. The reader is referred to the article by

\section{Transitional millisecond pulsars}

According to the classical recycling picture $[13,14,15]$, the switch on of a millisecond radio pulsar powered by the rotation of its magnetic field should occur only after the Gyr-long mass accretion phase in a LMXB has ceased and the pulsar wind 


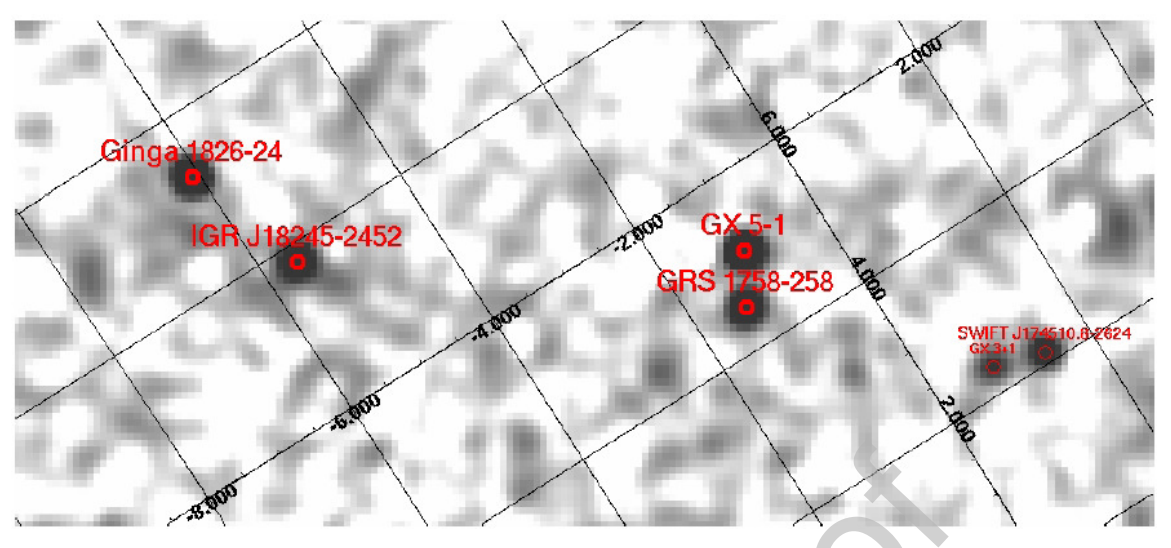

Figure 9: Mosaic of the IBIS/ISGRI field around IGR J18245-2452 at the time of its discovery in 2013 [37].

pressure becomes dominant. However, the possibility that a source could swing between a radio pulsar behaviour and a LMXB regime also over much shorter timescales (days to weeks) had been proposed already a few years before AMSPs were actually discovered [127, 128, 129]. When the X-ray luminosity of a transient LMXB drops below $10^{32} \mathrm{erg} \mathrm{s}^{-1}$ at the end of an outburst, the magnetospheric radius of a $\sim 10^{8} \mathrm{G}$ NS spinning at a period of a few milliseconds expands beyond the light cylinder radius of the pulsar, $r_{L C}=71.6\left(P_{s} / 1.5 \mathrm{~ms}\right) \mathrm{km}$, where $P_{s}$ is the pulsar spin period. A radio pulsar powered by the rotation of its magnetic field could then eject the residual disk matter and power the emission of the binary in quiescence. The discovery that AMSPs were weakly magnetized X-ray transients which dropped to an X-ray lumi345 nosity of $10^{31}-10^{32} \mathrm{erg} \mathrm{s}^{-1}$ during quiescence (see Sec. 2.2) was compatible with such a picture [130]. Furthermore, the optical luminosity of quiescent AMSPs turned out to be too large to be compatible with reprocessing of such a faint X-ray emission, suggesting it was instead due to reprocessing of the more powerful spin-down energy [131]. The rate at which AMSPs spin down during quiescence [132] and the fast and complex orbital evolution $[132,133]$ were also very similar to those observed from the so-called redback millisecond radio pulsars $[19,134]$. However, deep searches for radio pulsations from AMSPs in quiescence were not successful, even when conducted at high radio frequencies ( 5 and $8 \mathrm{GHz}$ ) at which free-free absorption is less important $[46,135,136]$.

The quest for sources switching back and forth between a radio pulsar and an accretion powered phase finally succeded when the radio pulsar PSR J1023+0038 showed indications of a past accretion disk activity [137], and eventually with the discovery of IGR J18245-2452, an AMSP that is detected as a radio pulsar during quiescence [37]. Dubbed transitional millisecond pulsars (see [12] and Papitto, in prep., for reviews), these sources are crucial to investigate how the interaction between the NS magnetosphere and the disk in-flow determines the pulsar emission regime. INTEGRAL played a crucial role in the recent discovery of this class mainly thanks to its all-sky monitoring capabilities. On one hand it detected the onset of the outburst of 


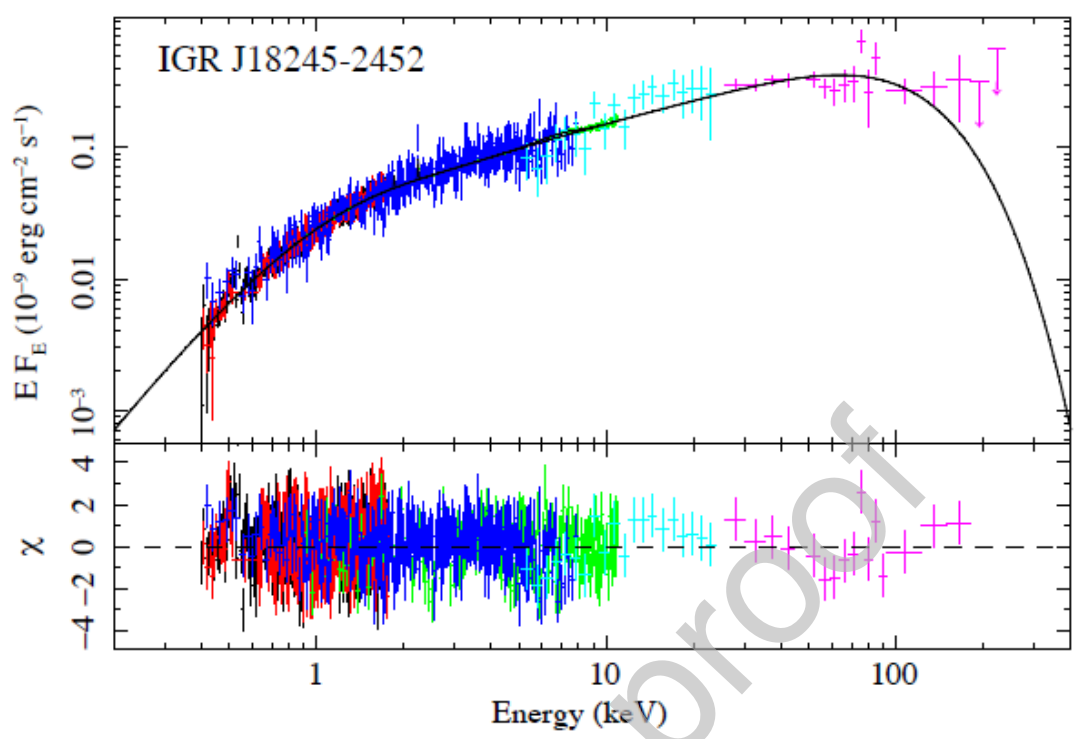

Figure 10: The unfolded absorbed broad-band spectrum of IGR J18245-2452 observed during the 2013 Xray outburst, fitted with a Comptonization compPs model (black line). Data points from XMM-Newton/RGS (red and black points, 0.4-1.8 keV), XMM-Newton/EPIC-pn (green points, 0.9-11 keV), Swift/XRT (blue points, $0.4-8 \mathrm{keV}$ ), INTEGRAL/JEM-X (light blue points, $5-25 \mathrm{keV}$ ) and INTEGRAL IBIS/ISGRI (magenta points, 22-250 keV) were included. The bottom panel shows residuals with respect to the best fit model. Figure from [39].

IGR J18245-2452 [138]; on the other, its catalogue included steady and relatively faint hard sources which were later identified as candidate transitional millisecond pulsars through multi-wavelength follow-up observations [139, 140].

\subsection{Swinging between accretion and rotation-powered states, IGR J18245-2452}

In 2013, IBIS/ISGRI discovered the transient IGR J18245-2452 in the globular cluster M28 [138, see Fig. 9]. The luminosity observed from the transient $\left(3 \times 10^{36} \mathrm{erg}\right.$ $\mathrm{s}^{-1}$ at a distance of $5.5 \mathrm{kpc}$ ) suggested that it was powered by mass accretion in a binary system. Subsequent observations of type-I X-ray bursts both by INTEGRAL [39] and Swift [37] identified the compact object in the binary as a NS. Finally, a coherent Xray periodicity at the $3.9 \mathrm{~ms}$ spin period of the NS was detected thanks to high-time resolution XMM-Newton observations; the spin and orbital parameters of this newlydiscovered AMSP were the same as those of a radio pulsar detected a few years before when the X-ray source was in quiescence, so unveiling the transitional nature of the system [37].

IBIS/ISGRI and JEM-X observations measured the properties of this transitional pulsar in the accretion phase and allowed to put it in the context of AMSPs [39]. The observed spectral energy distribution (see Fig. 10) was dominated by a power law component with a photon index of $\Gamma=1.32(1)$ and a cut-off energy of $122_{-16}^{+21} \mathrm{keV}$. Inter- 

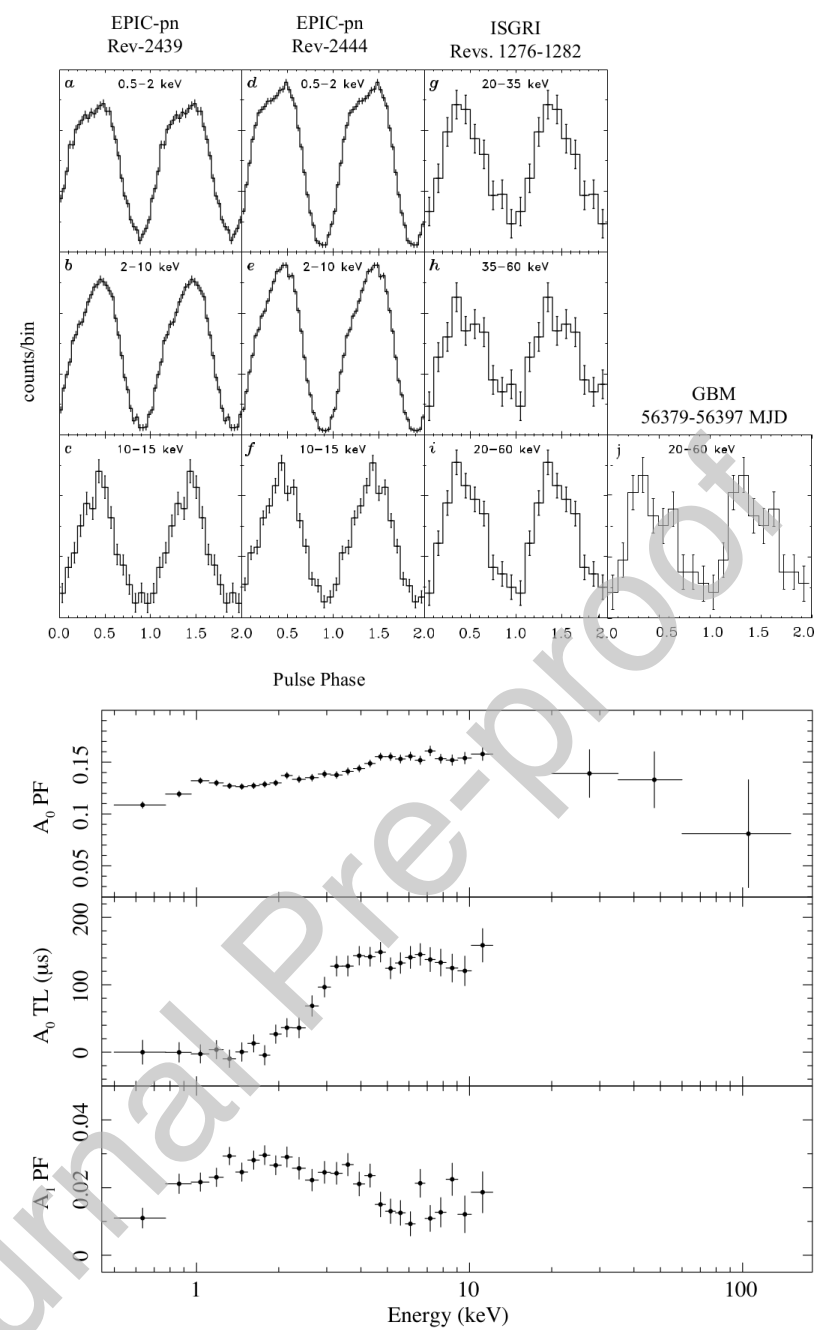

Figure 11: Top panel: pulse profiles of IGR J18245-2452 observed by XMM-Newton/EPIC-pn (panels a)-f), INTEGRAL IBIS/ISGRI (panels g)-i) and Fermi/GBM (panel j); bottom panel, from top to bottom: pulsed fraction of the first harmonic, hard phase/time lags, and pulsed fraction of the second harmonic. Reproduced from [39].

preted in terms of Comptonization of soft photons radiated from the NS hot spots, the spectral energy distribution measured from IGR J18245-2452 was the hardest among AMSPs. X-ray pulsations were detected by IBIS/ISGRI up to $60 \mathrm{keV}$, with a pulsed fraction of $\sim 10 \%$, compatible with the values detected at low energies (see the top panel of Fig. 11). The high energy pulses of IGR J18245-2452 lagged behind the pulses detected at soft X-rays by up to $150 \mu$ s (see the middle panel of Fig. 11). This 
was normally not observed in other AMSPs (see Sec. 2.4) and was ascribed to a peculiar energy dependence of the emission pattern of the hot spots on the NS surface [39].

Type-I X-ray bursts observed by INTEGRAL and Swift were all powered by ignition of Helium.

\subsection{X-ray sub-luminous transitional millisecond pulsars}

The month-long outburst observed in 2013 from IGR J18245-2452 has been the only bright accretion event observed from a transitional millisecond pulsar so far. Other transitional millisecond pulsars, such as PSR J1023+0038 [137] and XSS J12270-4859 [141], have persisted for years in an accretion disk state characterized by a much fainter X-ray luminosity $\left(L_{X} \simeq 5 \times 10^{33} \mathrm{erg} \mathrm{s}^{-1}\right.$ ), variable among two roughly constant levels (dubbed high and low modes) and frequent flares [142, 143, 144, 145]. This peculiar accretion disk state is also characterized by a variable, bright continuous radio emission $[146,147]$ and by an unexpected gamma-ray $(\sim \mathrm{GeV})$ emission with a power roughly comparable to that observed in the X-ray band [148, 149]. X-ray pulsations were detected only in the high mode $[150,151]$, simultaneously to unexpectedly bright optical pulses [152, 153].

The ability of IBIS/ISGRI in detecting faint, quasi persistent hard X-ray sources was crucial to identify such transitional pulsars in this enigmatic state. The case of XSS J12270-4859 is illustrative. A program of optical spectroscopy of unidentified INTEGRAL sources showed the presence in its spectrum of broad, double-peaked emission lines originating from an accretion disk [154]. While such a spectrum hinted at a cataclysmic variable, the presence of a gamma-ray counterpart detected by the Fermi/LAT 410 instrument suggested an atypical low-mass X-ray binary instead [142, 155, 156]. The IBIS/ISGRI spectrum was described by a power law with a hard photon index $(\Gamma=1.3)$ extending up to $100 \mathrm{keV}$ without a detectable cut-off [142]. The continuous detection by IBIS/ISGRI showed that the source could persist in such a state for at least a decade, possibly powered by a millisecond pulsar that ejects disk mass through the propeller

415 effect $[157,158]$ or accreting material at a very low rate $[159,160]$. The transitional nature of the source was eventually demonstrated when its disk disappeared [141] and it switched on as a radio pulsar [161].

Cross referencing the INTEGRAL IBIS/ISGRI catalogue with that of the Large Area Telescope (LAT; $20 \mathrm{MeV}$ - $300 \mathrm{GeV}$ ) aboard the Fermi Gamma-ray Space Tele420 scope [162] turned out to be one of the most effective techniques to identify strong candidate transitional millisecond pulsars in such a faint and peculiar accretion disk state (see Fig. 12), and allowed the identification of the candidates IGR J04288-6702 [139] and IGR J11098-6457 [140]. Follow-up observations of these two sources at optical and soft X-ray energies hinted at similar time variability properties as those observed from the other known transitional millisecond pulsars. However, their transitional nature is yet to be firmly established, waiting for a switch to the radio pulsar state hopefully in the near future.

\section{Rotation-powered pulsars}

Before the launch of INTEGRAL, highly magnetized $\left(B \simeq 10^{11}-10^{12} \mathrm{G}\right)$ rotationpowered pulsars were already known to emit steadily from radio frequencies up to 

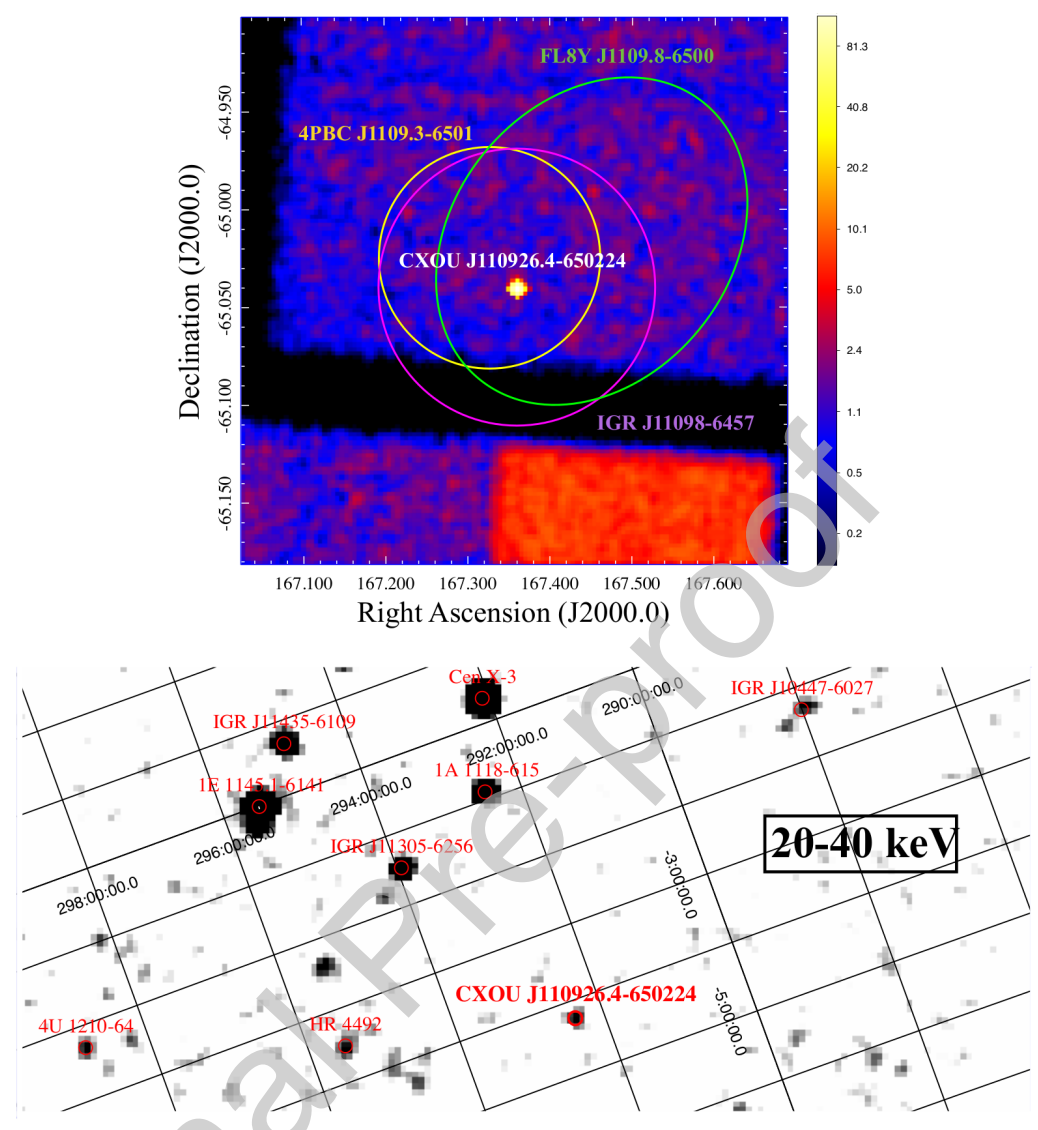

Figure 12: Top panel: Chandra/ACIS-I 0.3-8 keV image of the field around CXOU J110926.-650224 with the error circles of the counterparts from INTEGRAL (IGR J11098-6457, 20-40 keV, magenta line) Swift/BAT (4PBC J1109.3-6501, 15-150 keV, yellow line) and Fermi/LAT (FL8Y J1109.8-6500, $100 \mathrm{MeV}-300 \mathrm{GeV}$, green line) catalogues. Bottom panel: INTEGRAL IBIS/ISGRI 20-40keV mosaic image of the field around IGR J11098-6457; Figure from [140].

high-energy $\gamma$-rays. The Compton Gamma-Ray Observatory (CGRO) had increased the number of detected high-energy $(E \geq 100 \mathrm{MeV}) \gamma$-ray pulsars to a total of eight (see [163] for a review). However, the timing signatures and spectral shapes were only measured for three pulsars in the energy band from $\sim 20 \mathrm{keV}$ to $\sim 5 \mathrm{MeV}$ (or higher): the Crab pulsar (PSR B0531+21), PSR B1509-58 and the Vela pulsar (PSR B083345). This very small sample exhibited very different characteristics. The Crab and Vela pulsars showed double-peaked pulse profiles, while PSR B1509-58 showed a broad single pulse. The latter had a spectral shape peaking in luminosity at $\mathrm{MeV}$ energies, vastly different from that of the Crab [164]. The Vela pulsar was only marginally detected at hard X-ray/soft gamma-ray energies but appeared to be the strongest pulsar at 
$\mathrm{GeV}$ energies, where it reached maximum luminosity with a spectral shape completely different from both the Crab and PSR B1509-58 [165].

Before the launch of INTEGRAL, high-energy non-thermal emission from highly magnetized rotating NSs was believed to originate from particle acceleration along the open magnetic field lines in the pulsar magnetosphere. The different competing models could be grouped in two categories, the so-called Polar Cap (PC) models and Outer Gap (OG) models. In the PC scenario charged particles (mainly $e^{+} e^{-}$) are accelerated along the open field lines in the vicinity of the magnetic poles. Subsequent cascade processes (starting with curvature radiation or inverse Compton scattering) give rise to the emergent high-energy spectrum (e.g. [166]). In the OG scenario the acceleration of charged particles and production of high-energy radiation takes place in charge depleted gaps between the null-charge surface and the light cylinder above the last closed field lines (e.g. $[167,168])$. It was also predicted that due to their quick rotation, millisecond pulsars should be fairly strong high-energy gamma-ray emitters [169].

With the launch in June 2008 of Fermi, the LAT produced ground-breaking results at high-energy $\gamma$-rays. The number of detected $\gamma$-ray pulsars increased from eight to $\sim 250$ (status in 2019), including 90 millisecond pulsars (MSPs). These numbers can be compared to the seven pulsars securely detected above $100 \mathrm{MeV}$ with the Energetic Gamma Ray Experiment Telescope (EGRET) aboard CGRO and the single recycled MSP J0218+4232, likely detected above $100 \mathrm{MeV}$ [170]. The number of $\gamma$-ray sources totals more than 5000 in the fourth Fermi catalog [162], including 239 identified pulsars and many candidate rotation-powered pulsars. These results stimulated also major developments in theoretical modelling of the high-energy emissions in pulsar magnetospheres. The new emission characteristics, e.g. spectral turnover at GeV energies, pulse morphologies, favoured OG models over PC models [171] and Slot Gap models [172, 173]. However, recent global particle-in-cell simulations of pulsar magnetospheres reveal that most particle acceleration occurs in and near the current sheet beyond the light cylinder and the separatrices [174, 175, 176, 177, 178, 179].

INTEGRAL promised for the first time fine imaging and accurate localization of hard-X-ray $/ \gamma$-ray sources, with good timing and good sensitivity over the broad energy range from $3 \mathrm{keV}$ to $\sim 10 \mathrm{MeV}$. However, the predictions on the expected results for rotation-powered pulsars over this energy window were very uncertain. They relied on uncertain interpolations performed on a handful of radio pulsars between the spectra of pulsed emission measured at higher $\gamma$-ray energies and at soft X-ray energies, or on extrapolations of spectra measured only at lower X-ray energies. As expected, the strong Crab pulsar emission could be used as a calibration source, and studied in more detail at hard X-rays. However, the next-in-flux known hard X-ray pulsar, PSR B150958 , whose hard X-ray emission had been studied earlier using the instruments aboard CGRO [164] and the Italian/Dutch mission BeppoSAX [180], was already a factor 30-50 times weaker than the Crab in the $20-100 \mathrm{keV}$ band. The Vela pulsar was even fainter, with a hard X-ray luminosity a thousand times lower than the Crab. Using data from the RXTE, hard X-ray timing and spectral properties had been also reported for the Crab-like pulsar in the Large Magellenic Cloud (LMC), PSR B0540-69 [181], which turned out to be $\sim 250$ times weaker than that of the Crab in the hard X-ray domain. For the recycled rotation-powered pulsars, the MSPs, the prospects for detections with INTEGRAL were even smaller. Three MSPs, PSR B1821-24, PSR J0218+42323, and 
PSR B1937+21, had been reported to emit non-thermal emission at X-ray energies with very hard spectra with power-law indices $\Gamma \sim 1.1$. X-ray pulsations were detected up to $20 \mathrm{keV}$ for PSR B1821-24 [182] and PSR J0218+4232 [183], and up to $25 \mathrm{keV}$ orders of magnitude weaker than that of the Crab at $20 \mathrm{keV}$.

Given the expected low INTEGRAL count rates of the weak pulsed emission, it is no surprise that in the first decade of the INTEGRAL mission, most analyses did not address the pulsed emission but exploited the imaging capabilities in analyses of the total emission from pulsars and their Pulsar Wind Nebulae (PWNe). Only later, first detections at hard X-rays were reported and the total spectra (pulsar + PWN) discussed. The detection of emission up to $200 \mathrm{keV}$ from PSR B1509-58 in SNR MSH 15-52 [185] was followed by the presentation of the spatial and spectral properties of just the unpulsed emission in the $20-200 \mathrm{keV}$ band [186]. PSR B0540-69/ SNR 0540-693 in the LMC was first detected up to $100 \mathrm{keV} \mathrm{[187],} \mathrm{later} \mathrm{up} \mathrm{to} 200 \mathrm{keV}$, while significant pulsations with a high duty cycle were visible up to $100 \mathrm{keV}$ [188]. For PSR J16175055 near RCW 103 (18 - $60 \mathrm{keV} \mathrm{[189])} \mathrm{and} \mathrm{later} \mathrm{PSR} \mathrm{J1811-1925} \mathrm{in} \mathrm{G11.2-0.3} \mathrm{(up}$ to $200 \mathrm{keV}[190])$ the total spectra could be discussed for the first time. In addition, with INTEGRAL IBIS/ISGRI PSR J0537-6910 was detected in the $20-60 \mathrm{keV}$ band in a very deep survey of the LMC region [191].

The above summary indicates that very long INTEGRAL exposures were required to increase the sample of pulsars for which pulsed emission could be detected at hard X-rays. Similar to the case of AMSPs (see Sec. 2.4), the low pulsed-count rates in the INTEGRAL window required timing analyses to rely on pulsar ephemerides determined in radio monitoring observations or in the X-ray band below $\sim 10 \mathrm{keV}$ where instruments such as the PCA aboard RXTE were sufficiently sensitive to allow for measurements of pulsar ephemerides. The latter was required when the pulsars were not detected in the radio band. Once these timing solutions were determined at lower energies, phase folding of the event arrival times measured with the INTEGRAL instru515 ments could be performed to search for the pulsed signals at hard X-rays. A complete high-energy overview of the soft $\gamma$-ray pulsar population was published with a catalog containing 18 rotation-powered (non-recycled) pulsars with pulsed emission detected in the hard X-ray band above $20 \mathrm{keV}$ [192]. Surprisingly, most of these pulsars were not detected by Fermi at high-energy $\gamma$-rays. This catalog and the characteristics of 520 this sample will be addressed at the end of this section. First, the INTEGRAL results from studies of the Crab pulsed emission will be presented, followed by the important results from polarization measurements.

\subsection{The Crab pulsar}

Early in the mission, the first INTEGRAL results were published for the archetypical Crab pulsar with a verification of the absolute timing capabilities of all high-energy instruments [8, 193], JEM-X, IBIS/ISGRI and SPI. It was shown that the X-ray main pulse is leading the radio pulse in phase by $285 \pm 12 \mu$ s (IBIS/ISGRI) and $265 \pm 23 \mu \mathrm{s}$ (SPI) [8] (statistical errors only), values that are more accurate than those reported earlier. Using six years of SPI telescope data (total exposure $\sim 4 \mathrm{Ms}$ ) these values were confirmed $(275 \pm 15 \mu \mathrm{s})$ [194] for the hard X-ray band 20-100 keV. More interestingly, it was shown that the delay between the radio and X-ray signals varies in the $20-300$ 
$\mathrm{keV}$ range. Namely, the delay was reported to be $310 \pm 6 \mu$ s in the $3-20 \mathrm{keV}$ soft $\mathrm{X}$-ray band from an analysis of $R X T E$ data [194].

A coherent high-energy picture of the Crab nebula and pulsar spectra from soft $535 \mathrm{X}$-rays up to high-energy $\gamma$-rays had been published shortly before the INTEGRAL launch [195], including a pulse-phase-resolved spectral analysis performed in seven phase slices over the $0.1 \mathrm{keV}-10 \mathrm{GeV}$ energy band. In this high-energy picture of the Crab, data from the four narrow-field instruments aboard BeppoSAX: LECS, MECS, HPGSPC and PDS covered energies up to $300 \mathrm{keV}$ (see [195] for references). At higher $540 \gamma$-ray energies, data were used from COMPTEL and EGRET aboard CGRO. Early in the INTEGRAL mission, an accurate phase-resolved (now in 50 bins) spectral analysis for the Crab pulsar over the energy range 3-500 keV was achieved with multiple Crab calibration observations with JEM-X, IBIS/ISGRI \& PICsIT and SPI, characterizing in detail the curved spectral shape over this energy range [196]. The combination of INTEGRAL timing and spectral results with those from the previous BeppoSAX and $C G R O$ missions were input for a multi-component model for the broad-band emission of the Crab pulsar from the optical band to high-energy $\gamma$-rays [197].

\subsection{Joint optical - $\gamma$-ray polarisation measurements with INTEGRAL}

Although a relatively weak radio pulsar and a strong emitter at X and $\gamma$-ray energies, the Crab pulsar is the brightest of all the known pulsars at optical wavelengths and consequently has been extensively studied. The pulsar's spin-down energy powers its surrounding nebula, which radiates at all electromagnetic frequencies from the radio band to TeV $\gamma$-rays [198]. Our understanding of the high-energy emission process in pulsars is still very incomplete. However, polarisation observations can begin to unravel this conundrum through geometric considerations. Harding and Kalapotharakos [199] have modelled the high-energy polarised emission from optical to $\gamma$-ray wavelengths. They predict a polarisation degree and polarisation angle which depends upon location and specifically whether the radiation originates from inside or outside the pulsar's light cylinder. Matching the polarisation profile can give a unique restriction 560 on the location of the production of high-energy emission [200].

The Crab nebula was one of the first objects outside of the Solar System to have detectable X-ray polarisation [201]. This was followed by the first detection of $\gamma$ ray polarisation using INTEGRAL [202, 203]. Optical polarised emission from the nebula and pulsar have been described by a number of authors [204, 205, 206, 207, 208]. Phase-resolved observations showed a change in polarisation consistent in shape with a beam of synchrotron radiation coming from both poles of an orthogonal rotator. Słowikowska et al. [205, 209] found the presence of a highly polarised continuous component which most likely corresponds to the nearby bright synchrotron emitting knot [207], also known as inner-knot, located 0.65 arcsec to the southest of the pulsar 570 [210].

Optical and X-ray observations showed spatial and some flux variability of the inner nebula [211]. Indeed this had been observed in some early optical studies [212]. However, the flux from the whole nebula was expected to be constant at the level of a few percent $[213,214]$ and as such was often used as a standard candle calibration source. However since 2008, strong $\gamma$-ray flares have been observed at a rate of about 1 per year by the Agile and Fermi $\gamma$-ray telescopes [215, 216, 217]. Around these $\gamma$-ray 


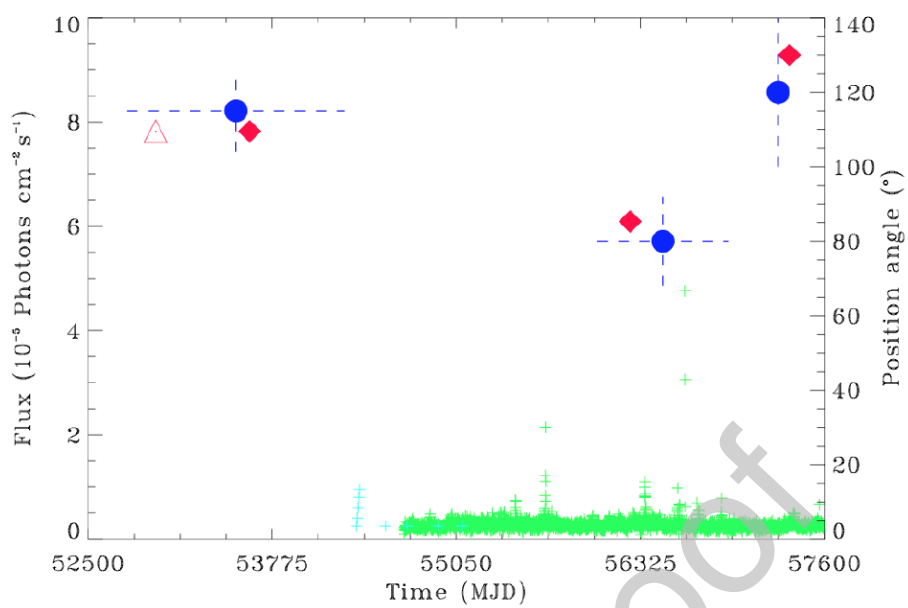

Figure 13: Galway Astronomical Stokes Polarimeter (GASP, [219], open triangle and filled diamonds) and INTEGRAL observations of the polarisation position angle of the Crab (blue filled circles; [208, 220]). Green and cyan points show the flux observed by Fermi and AGILE, respectively. More recent Astrosat measurements [221] in the $0.1-0.38 \mathrm{MeV}$ band indicate a polarisation angle of $(143.5 \pm 2.8)^{\circ}$ consistent with the trend observed by GASP and INTEGRAL. Figure taken from [208].

flaring events, there were no associated changes seen in the near-IR or X-ray fluxes [218].

Since the original INTEGRAL $\gamma$-ray observations in 2008, the Crab has been observed twice a year improving the statistics for determining the $\gamma$-ray polarisation [222]. This has allowed for comparisons between optical and $\mathrm{MeV} \gamma$-ray polarisation. In particular Moran et al. [208] looked for any correlated changes in the polarisation at the two wavebands. Changes in the polarisation angle seemed to be correlated, albeit only at the $2.5 \sigma$ level (see Fig. 13). Whether this is indicative of reconnection or other events associated with $\gamma$-ray flares is not proven. Other suggestions include magnetoBremsstrahlung emission which explain the lack of variability at lower photon energies [218]. More correlated observations simultaneous with a flare are required to address this problem, either looking at flux and polarisation changes in the nebula and/or in the immediate vicinity of the pulsar.

4.3. The soft $\gamma$-ray pulsar catalog

Despite the weakness of the pulsar signals in the INTEGRAL hard X-ray/soft $\gamma$ ray band, significant progress was achieved when the X-ray observatories Chandra, RXTE, XMM-Newton, Suzaku, INTEGRAL and later NuSTAR, discovered weak energetic point sources at soft/medium X-rays $(0.1-10 \mathrm{keV})$ in young supernova remnants, often detected at radio frequencies or in location-error boxes of unidentified CGRO EGRET/Fermi LAT ( $\geq 100 \mathrm{MeV})$, INTEGRAL IBIS/ISGRI (20-300 keV) or H.E.S.S./VERITAS/MAGIC $(\geq 30 / 100 \mathrm{GeV})$ sources. The pulsed signals of these new point sources could be identified at soft $\mathrm{X}$-rays or in the radio band. Once the timing 


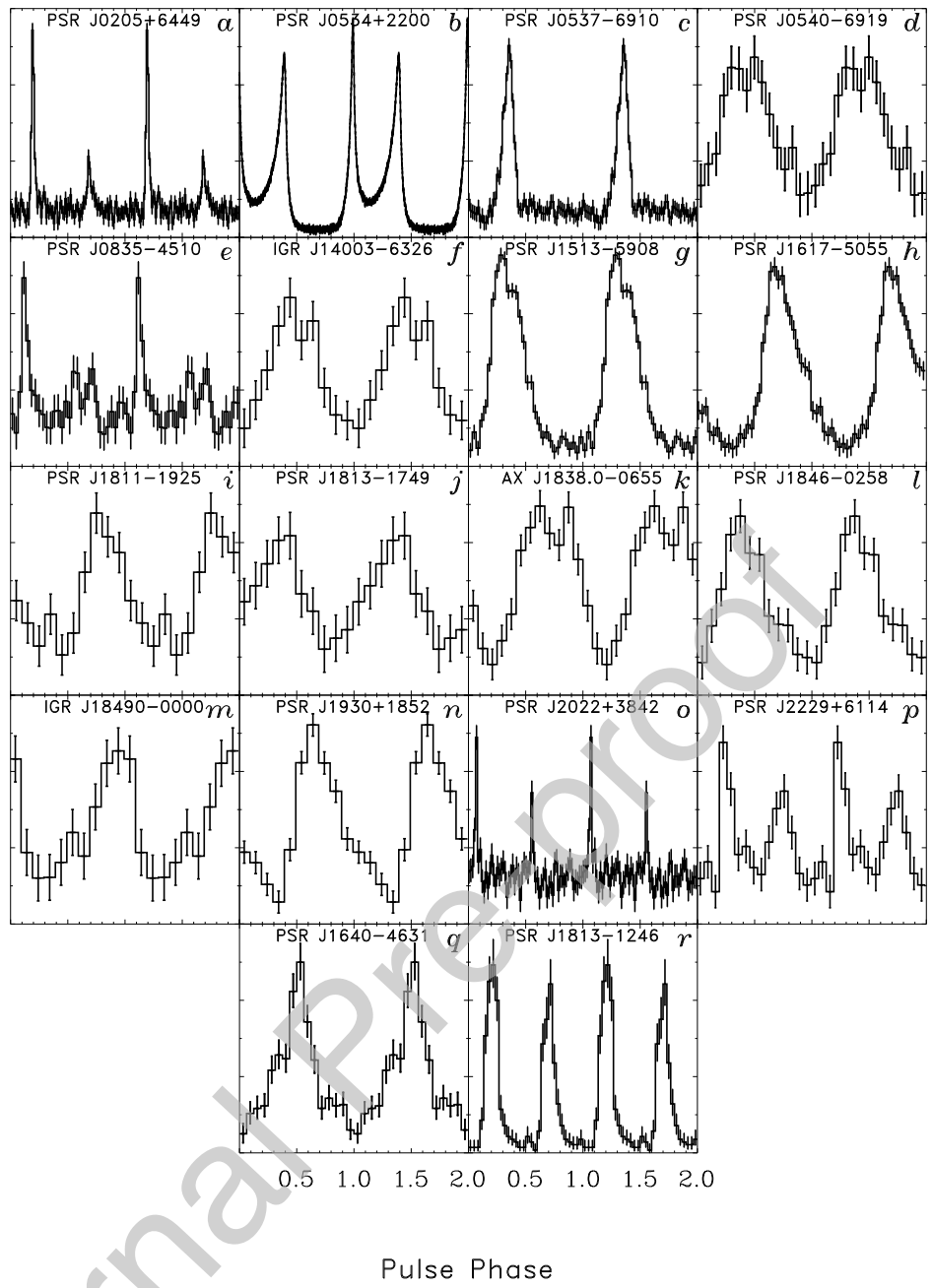

Figure 14: The pulse shapes of all soft $\gamma$-ray pulsars in the catalog [192]. The profiles are measured either with INTEGRAL IBIS/ISGRI or RXTE PCA or HEXTE. Exceptions are PSR J1640-4631 (NuSTAR) and PSR J1813-1246 (XMM-Newton EPIC).

solutions were determined below $10 \mathrm{keV}$, phase folding of the event arrival times measured with RXTE PCA and HEXTE and INTEGRAL IBIS/ISGRI could be performed to search for the pulsed signals above $20 \mathrm{keV}$. The imaging capabilities of IBIS/ISGRI allowed in addition the detection and spectral characterization of the total emission (pulsar plus PWN), often up to energies above $100 \mathrm{keV}$. However, very long exposures were required to obtain secure detections, collecting over many years exposures each time a source was in the field-of-view of INTEGRAL and/or RXTE. This approach led to an INTEGRAL/RXTE soft $\gamma$-ray pulsar catalog containing 18 pulsars for which nonthermal pulsed emission has been securely detected at hard X-rays/soft $\gamma$-rays above $20 \mathrm{keV}$ [192]. That paper summarizes the history of the detection of each pulsar in different bands of the electromagnetic spectrum, and the X-ray timing and spectral 
characteristics. Below, we will present the role of INTEGRAL in the discovery and/or characterization of a few pulsars, followed by a comparison of the sample of hard Xray/soft $\gamma$-ray pulsars with those in the second Fermi pulsar catalog [223].

\subsubsection{IGR J18490-0000, IGR J14003-6326 and IGR J11014-6103 from INTEGRAL sources to pulsars}

615

Three pulsars were originally discovered as X-ray point sources by INTEGRAL in deep IBIS/ISGRI mosaic images, namely IGR J18490-0000/PSR J1849-0001 [224], IGR J14003-6326/PSR J1400-6325 [225] and IGR J11014-6103/PSR J1 101-6101 [226]. Follow-up observations outside the INTEGRAL band led to their identification as rotationpowered pulsars.

Multiple follow-up observations of IGR J18490-0000 revealed the presence of a $\mathrm{TeV}$ source with HESS, a soft X-ray counterpart with Swift-XRT and XMM-Newton and, finally [227], the 38.5-ms X-ray pulsation and a weak PWN with Chandra (for further references and derived (hard) X-ray characteristics see [192]), while no radio counterpart was detected with the GMRT. The discovery of IGR J14003-6326 triggered similar follow up observations, notably with Chandra, revealing the presence of a point source plus a PWN and a SNR [228]. The latter reference also reported the detection of a 31.2-ms pulsar with RXTE PCA. Improved (hard) X-ray characteristics are given with the catalog [192]. Both these new hard X-ray pulsars were not detected by Fermi LAT. For the strongest of the two (PSR J1849-0001) one can conclude that the non-detection by the LAT means that maximum luminosity is reached at $\mathrm{MeV}$ energies.

IGR J11014-6103 and its surroundings were studied in great detail in X-rays, first by using all available archival X-ray data [229] and subsequently with a dedicated Chandra observation [230]. It was shown that the putative pulsar counterpart is moving away from SNR MSH 11-61A, located at 11 arcmin from the X-ray point source. The latter was shown to have a hard spectrum in the $2-10 \mathrm{keV}$ band (power-law photon index $\Gamma=1.1 \pm 0.2$ ). Finally, XMM-Newton observations revealed the X-ray pulsations $(62.6 \mathrm{~ms})$ [231] and allowed the characterisation of the pulsed-emission X-ray spectrum up to $10 \mathrm{keV}$ [192]. This pulsar is an excellent candidate to be detected at higher $\mathrm{X}$-ray energies as a soft $\gamma$-ray pulsar.

\subsubsection{AX J1838.0-0655 / PSR J1838-0655, another 'MeV' pulsar}

An interesting discovery turned out to be the detection of soft $\gamma$-ray emission up to $300 \mathrm{keV}$ from the ASCA source AX J1838.0-0655 using INTEGRAL IBIS/ISGRI data [232]. Its location made an association with TeV source HESS J1837-069 [233] plausible, suggesting a pulsar/PWN origin, later confirmed using RXTE PCA data (23.4 kyr-old pulsar with period $70.5 \mathrm{~ms}$ ) [234, 235]. This young pulsar turned out to be the third pulsar in hard-X-ray flux after the Crab pulsar and PSR B1509-58, without detections in the radio and Fermi high-energy $\gamma$-ray bands. Analysis of RXTE/HEXTE and INTEGRAL IBIS/ISGRI data revealed X-ray pulse profiles up to $\sim 150 \mathrm{keV}$ with pulse and spectral shapes similar to that of PSR B1509-58 [192]. This pulsar turned ${ }_{650}$ out to be another prototype example of a pulsar with a spectrum reaching maximum luminosity at $\mathrm{MeV}$ energies. 

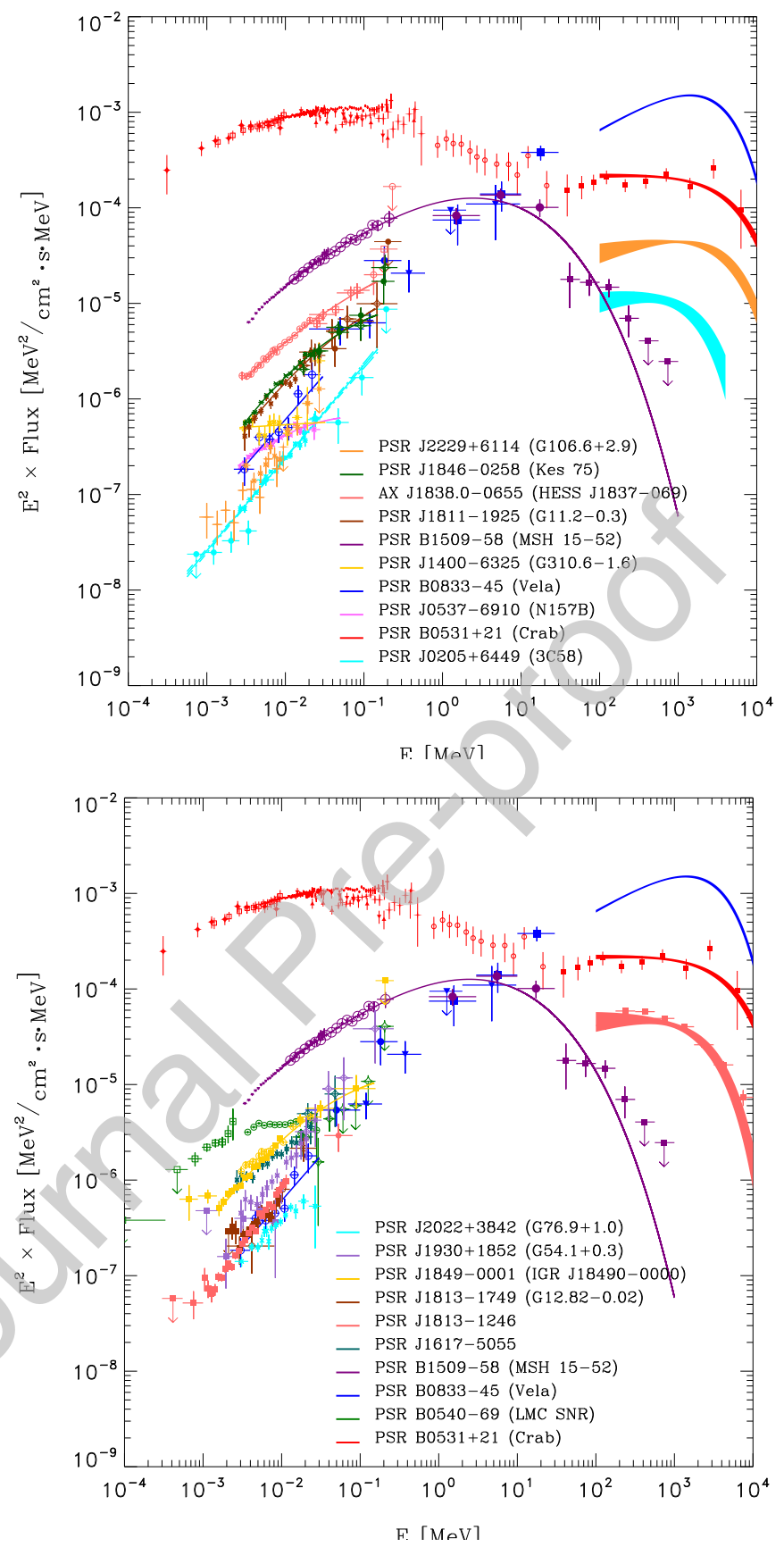

Figure 15: The high-energy pulsed emission spectra for 17 of the 18 detected soft $\gamma$-ray pulsars from $0.1 \mathrm{keV}$ $-10 \mathrm{GeV}$ summarized in two panels. The spectra of PSR B0531+21 (Crab), PSR B0833-45 (Vela) and PSR B1509-58 are shown in both panels for reference purposes. The pulsed spectrum of PSR J1640-4631 is not shown, because its total spectrum has been reported up to hard X-rays but its pulsed spectrum just up to $25 \mathrm{keV}$. Figure taken from [192]. 


\subsubsection{PSR J1846-0258 showing magnetar-like behaviour}

A very intriguing high-B-field $\left(4.9 \times 10^{13} \mathrm{G}\right)$ pulsar is PSR J1846-0258, a relatively slow $(P \sim 324 \mathrm{~ms})$ radio-quiet pulsar, with the smallest characteristic age $(\tau \sim 723 \mathrm{yr})$

all known pulsars. It is located in the centre of SNR Kes75, showing up as a bright hard X-ray source surrounded by a diffuse PWN. INTEGRAL detected point-source emission up to $\sim 200 \mathrm{keV}$ and pulsed emission up to $\sim 150 \mathrm{keV}$ (see [236] and references therein). Most surprisingly, this pulsar showed magnetar-like behaviour during 2006 June 7-12 with an increase by $\sim$ a factor five in luminosity due to a radiative outburst in soft X-rays lasting 55 days and accompanied by five magnetar-like bursts $[237,238]$. The onset of the radiative event was accompanied by a major spin-up glitch of the pulsar [236]. IBIS/ISGRI could study the evolution of the total non-thermal flux (PSR J1846-025 + Kes-75) during the years 2003-2006 before and after the outburst. The pulsar was found to be stable in X-rays before the spin-up glitch with a power-law spectrum (index $\Gamma=1.80 \pm 0.06$ ). During the outburst the hard X-ray flux increased by $\sim 50 \%$, the spectral shape remained the same, and after one year the non-thermal emission was back to its pre-outburst values. The X-ray pulse profile measured by IBIS/ISGRI, PCA and HEXTE, was a broad single asymmetric pulse that did not vary in shape over the 3-150 keV energy range and, remarkably, did not change during the magnetar-like outburst, nor did its non-thermal spectral shape (power-law index $\Gamma$ 1.2). The accurate IBIS/ISGRI measurement of the total and pulsed spectra showed that the pulsed fraction approaches $100 \%$ around $150 \mathrm{keV}$ [236]. In its steady state, also this pulsar exhibits very similar timing and spectral characteristics as PSR B150958 with a spectrum reaching its maximum luminosity at $\mathrm{MeV}$ energies, confirmed with the recent detection with Fermi LAT of a very weak pulsed emission between 30 and $100 \mathrm{MeV}$ [239].

\subsubsection{Hard X-ray pulsars, a distinct subset of the non-thermal population of rotation- powered pulsars}

The hard X-ray/soft $\gamma$-ray $(\mathrm{E} \geq 20 \mathrm{keV})$ pulsar population counts only 18 members [192], all non-recycled pulsars, compared to a total of 160 (status in 2019) nonrecycled pulsars detected by Fermi LAT above $100 \mathrm{MeV}$. As was expected, INTEGRAL could not detect the very weak pulsed emission from the recycled MSPs, even though Fermi had increased the number of MSPs detected above $100 \mathrm{MeV}$ from one to $\sim 60$. The question from the start of the INTEGRAL mission was: will INTEGRAL just detect/confirm the hard X-ray spectral tails of the high-energy $\gamma$-ray pulsars that reach their maximum luminosities at $\mathrm{GeV}$ energies and have predominantly narrow (double) pulse profiles, or will INTEGRAL provide new information on the total high-energy non-thermal pulsar population? In order to investigate this question, the characteristics of the 18 soft $\gamma$-ray pulsars were compared with those of the 77 non-recycled LATdetected pulsars in the Second Fermi Pulsar Catalog [192]. Surprisingly, it was found that the soft $\gamma$-ray pulsars are all fast rotators and on average $\sim 9.3$ times younger and $\sim 43$ times more energetic than the Fermi LAT sample (see also [240] for a recent assessment). The majority (11 sources) exhibits broad, structured single pulse profiles, and only six have double (or even multiple, Vela) pulses (see Fig. 14). Fifteen soft $\gamma$-ray pulsars show hard power-law spectra in the hard X-ray band and reach maximum luminosities typically in the $\mathrm{MeV}$ range (see Fig. 15). For only seven of the $18 \mathrm{soft}$ 
$\gamma$-ray pulsars pulsed emission has also been detected by the LAT, but 12 have a PWN detected at $\mathrm{TeV}$ energies. In conclusion, observations of rotation-powered pulsars at hard X-rays and $\mathrm{MeV} \gamma$-rays reveal a subset of the total high-energy pulsar population Xet (yet) be observed with Fermi at energies above $100 \mathrm{MeV}$. This distinct subsample was originally not taken into account in population studies based on the Fermi catalog. However, Torres [241, 242] presented recently a physical model for the nonthermal emission of pulsars above $1 \mathrm{keV}$ to fit the spectra of the $\gamma / \mathrm{X}$-ray pulsars along seven orders of magnitude over the INTEGRAL band up to the Fermi high-energy $\gamma$ rays. The spectra of all pulsars with detected non-thermal emission could be modeled with a continuous variation of only four model parameters, and it was proposed that their values likely relate to the closure mechanism operating in the accelerating region.

After the launch in June 2012 of NuSTAR (two orders of magnitude more sensitive than INTEGRAL in the energy band 3-79 keV) several of the rotation-powered pulsars in the soft $\gamma$-ray pulsar catalog have been observed. The published temporal and spectral characteristics were all confirmed, and so far no new entries to the pulsar catalog were reported. However, NUSTAR made some progress by detecting the non-thermal hard-X-ray pulsations of PSR B1821-24, PSR B1937+21 and PSR J0218+4232 for energies up to $\sim 50 \mathrm{keV}, \sim 20 \mathrm{keV}$ and $\sim 25 \mathrm{keV}$, respectively [243], and confirming the earlier reported hard X-ray spectra with photon indices $\Gamma \sim 1.1$. Still, INTEGRAL remains unique in its capabilities to image point sources (pulsars + PWNe) up to few hundred $\mathrm{keV}$, as well as to measure pulsed-emission from non-recycled pulsars above $80 \mathrm{keV}$.

\section{Magnetars}

Magnetars are isolated NSs defined by the fact that the main source powering their persistent and flaring emission is magnetic energy [244, 245]. Their external magnetic field is estimated to reach $10^{15} \mathrm{G}$, and their internal field might be even higher. Although we know only about two dozens of magnetars in the Galaxy and in the Magellanic Clouds, the extreme properties of this small class of objects make them partic725 ularly interesting as laboratories to study physical processes in high magnetic fields. They emit predominantly in the X-ray and soft $\gamma$-ray energy range, where they show a variety of variable phenomena, ranging from short bursts on sub-second timescales, to outbursts lasting several months.

Their magnetic field, much larger than in ordinary NSs, is likely produced thanks to a very short rotational period at birth, of the order of only 2-3 ms [246]. Magnetars have been invoked as central engines of gamma-ray bursts, able to power the prompt emission and/or part of the afterglow, as a possible explanation for the enigmatic Fast Radio Bursts, as well as potential sources of gravitational waves.

For a thorough description of the magnetar observational properties and of the theoretical models proposed to explain them we refer to several recent reviews [247, 248, 249, 250]. Here, we concentrate on the results obtained with the INTEGRAL satellite. We first describe the discovery of hard X-ray emission in magnetars, then we summarize the results concerning the short bursts, and, finally, we describe the observations of the only giant flare emitted by a magnetar after the INTEGRAL launch. 


\subsection{The discovery of persistent hard $X$-ray emission}

Although magnetars were first discovered as Soft Gamma-ray Repeaters (SGRs) through the detection of bursts in the hard X-ray band [251], until the launch of INTEGRAL their persistent emission had been observed only in the classical $\sim 1-10 \mathrm{keV}$ $\mathrm{X}$-ray range. At these energies, the persistent X-ray counterparts of the SGRs, as well 745 as the Anomalous X-ray Pulsars (AXPs, another class of X-ray sources [252] later recognized to be magnetars), are characterized by rather soft X-ray spectra. These spectra were usually fitted with the sum of a thermal component, with typical blackbody temperature of the order of $\sim 0.5 \mathrm{keV}$, and a steep power law with photon index $\Gamma$ ranging between $\sim 3$ and $\sim 4$ [253]. Therefore, the INTEGRAL discovery of persistent hard Xray emission from several magnetars was quite unexpected since a simple extrapolation of the X-ray spectra would lead to very small hard X-ray fluxes.

An INTEGRAL source with average flux of $7 \mathrm{mCrab}$ in the $60-120 \mathrm{keV}$ range and coincident with the AXP 1E 1841-045 in the Kes 73 supernova remnant was first reported in [224]. This discovery prompted an analysis of archival RXTE data ([254], see also [255] for a re-analysis of BeppoSAX data) that, thanks to the detections of pulsations up to $\sim 150 \mathrm{keV}$, confirmed that the hard $\mathrm{X}$-rays were indeed emitted by the magnetar and not by the supernova remnant. The pulsations were later found also in the IBIS/ISGRI data [256].

Hard X-ray spectral components were subsequently detected with INTEGRAL in other AXPs (1RXS 1708-4009 and 4U 0142+61 [257, 256, 258]), as well as in the two brightest SGRs: 1806-20 [259, 260, 261] and 1900+14 [262, 263]. As an example, we show in Fig. 16 the spectrum of $4 \mathrm{U} 0142+61$. All these magnetars are persistently bright sources, but in the following years INTEGRAL detected hard X-ray tails also in transient magnetars, such as SGR 0501+4516 [264] and 1E 1547.0-5408 [265, 266], when they went in outbursts.

The INTEGRAL results for the latter source are particularly interesting because they showed the appearance of a new transient component extending up to $150 \mathrm{keV}$ after the onset of the January 2009 outburst [266]. This pulsed component was shifted in phase with respect to the lower energy pulse profile and had a different spectral and flux evolution compared to that of the total hard X-ray emission.

These INTEGRAL observations, as well as further hard X-ray data obtained with other satellites (mainly Suzaku and NuSTAR [267, 268, 269]), have clearly shown that the magnetar hard X-ray tails extending up to $\sim 150-200 \mathrm{keV}$ are not a simple extrapolation of their lower energy emission. In fact, they are fitted by flatter power-laws

$775\left(\Gamma_{\gamma} \sim 0.5-2\right)$ and often show a pulse profile different from that seen below $10 \mathrm{keV}$ (see, e.g., Fig. 17). The pulsed flux is generally harder than the unpulsed one, causing an increase of pulsed fraction with energy. The upper limits in the MeV region $[256,258,270]$ imply a spectral turn-over of the hard components, which, nevertheless, contain an energy of the same order of that of the soft X-ray emission, or in some 780 cases even larger

While the soft X-rays are generally ascribed to thermal emission from the magnetar surface, modified by the effects of a strongly magnetized atmosphere, the hard X-ray tails are thought to originate from non-thermal particles in the magnetosphere (see, e.g., [271]), likely with an important contribution from resonant cyclotron scattering 


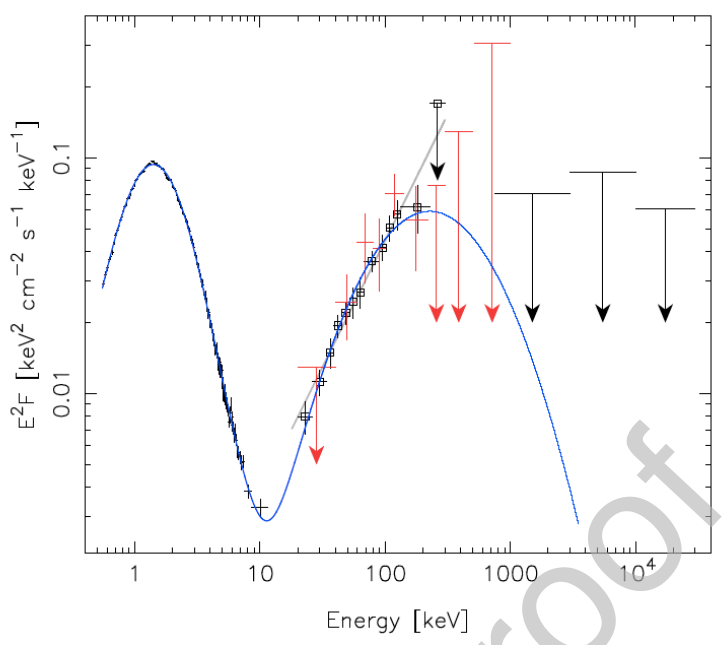

Figure 16: The broad band spectrum of $4 \mathrm{U} 0142+61$ (from [258]). The data below $10 \mathrm{keV}$ are from XMMNewton, while those above $20 \mathrm{keV}$ are from the INTEGRAL IBIS/ISGRI(black) and SPI (red) instruments. The upper limits in the $0.75-30 \mathrm{MeV}$ range are from COMPTEL. The blue line is a best fit with a phenomenological model consisting of the sum of log-parabolic functions, that clearly illustrates the different components at soft and hard X-ray energies.

[272]. In the twisted magnetospheres of magnetars, currents flow along bundles of closed field lines, which in turn can have a complicated geometry with time-dependent local structures. As a result, the computations of the emerging spectra [273, 274, 275], as well as their consistent comparison with the observational data [276, 277], were not simple. Nevertheless, this research field triggered by the INTEGRAL discovery offered in perspective a new important channel for the understanding of physical properties of magnetars and their emission processes.

\subsection{Bursts from soft gamma-ray repeaters}

Two magnetars have been particularly active during the first years of the $I N$ TEGRAL mission, leading to the detection of several bursts: SGR 1806-20 and 1E 1547.0-5408. Many of these bursts, as well as a few ones from other magnetars, occurred within the field of view of the IBIS/ISGRI instrument and were detected and localized in real time by the INTEGRAL Burst Alert System (IBAS, [278]). More recently, a particularly interesting result was obtained with the IBAS detection of a peculiar burst from the magnetar SGR 1935+2154 [279]. This burst was characterized by the simultaneous emission of an extremely bright radio pulse with properties similar to those of the fast radio bursts [280, 281].

A detailed analysis of the bursts from SGR 1806-20 was reported in [282] and [283]. Thanks to the high sensitivity of the IBIS/ISGRI imager in the $15-200 \mathrm{keV}$ range, it was possible to study for the first time the faint end of the luminosity distribution of the SGR bursts. Indeed, the faintest bursts observed in October 2003 had fluences as low as $2 \times 10^{-8} \mathrm{erg} \mathrm{cm}^{-2}$. Several bursts showed a significant spectral 


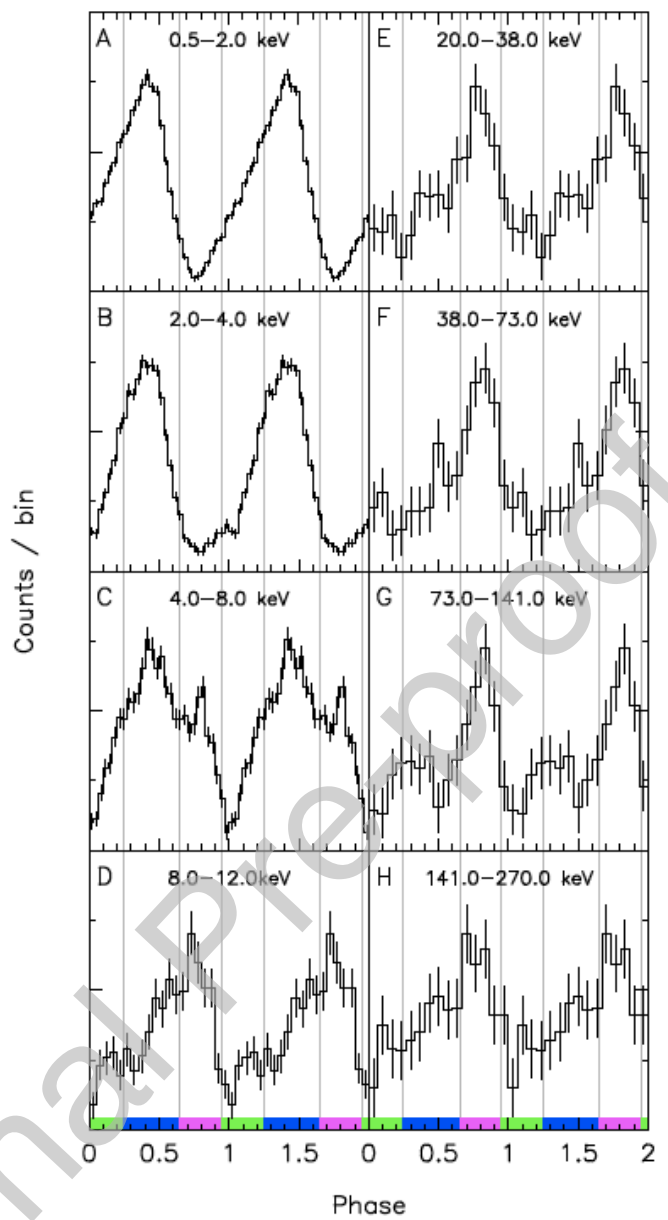

Figure 17: Pulse profiles of 1RXS 1708-4009 (from [270]) obtained in the soft X-ray band with XMMNewton (left panels) and in the hard X-ray band with INTEGRAL (right panels). Observations were not strictly simultaneous.

evolution in the hard X-ray range and some evidence for an overall anticorrelation between spectral hardness and flux was found with a time resolved spectral analysis of the whole sample of bursts [282]. This anticorrelation was later confirmed with the analysis of a larger sample of more than 200 bursts [283]. The INTEGRAL distribution of the burst fluences was found to be a power-law with index $(0.91 \pm 0.09)$, for fluences in the range between $3 \times 10^{-8}$ and $2 \times 10^{-6} \mathrm{erg} \mathrm{cm}^{-2}$. This rather flat slope implies that the integrated flux of fainter bursts below the detection threshold does not contribute significantly to the flux of the "persistent" hard X-ray emission.

1E 1547.0-5408 is a transient magnetar that exhibited three major outbursts: in June 2007, October 2008 and January 2009 [265]. During the latter outburst, this 
source emitted numerous short bursts reaching a particularly high rate on January 22, when more than 200 bursts were detected by INTEGRAL in a few hours [284]. Contrary to the case of SGR 1806-20, they showed a positive correlation between hardness and above $2 \times 10^{-4} \mathrm{erg} \mathrm{cm}^{-2} \mathrm{~s}^{-1}$ at $E>25 \mathrm{keV}$. Two of them lasted several seconds, and had tails modulated at the NS spin period of $2.1 \mathrm{~s}$. In particular, the time evolution of the burst shown in Fig. 18 resembled that of the giant flares. However, the energy released in this event was at most $\sim 10^{43} \mathrm{erg}$ (for $d=5 \mathrm{kpc}$ ), which is orders of magnitude smaller than that of the three magnetar giant flares observed to date.

The INTEGRAL results on 1E 1547.0-5408 triggered follow-up observations with other facilities. X-ray images obtained on 2009, January 23 and in the following two weeks with Swift and XMM-Newton showed the presence of three expanding rings around the source position [286], caused by scattering from relatively thin dust layers along the line of sight. By fitting the expansion rate of the rings it was possible to determine the time of the burst(s) responsible for the scattered X-ray radiation and it was found to be well in agreement with the period of highest bursting activity seen with INTEGRAL. Furthermore, with a spectral analysis of the scattered X-rays it was also possible to estimate a distance of 4-5 kpc for 1E 1547.0-5408.

A particularly interesting burst was discovered at 14:34:24 UTC of April 28 by the IBAS software, that automatically identified its origin from the transient magnetar SGR 1935+2154 and distributed a public alert after less than 10 seconds. This event was indepentently discovered at radio wavelengths $[280,281]$ and represents the first, and so far unique, SGR burst from which simultaneous radio emission has been detected. The INTEGRAL data obtained with IBIS, as well as those obtained by other high-energy satellites [287, 288], showed that its spectrum was harder than that of typical magnetar bursts. On the other hand, this event was not particularly luminous, with a 20-200 keV emitted energy of the order of $\sim 10^{39} \mathrm{erg}$ (assuming isotropic emission and a distance of $4.4 \mathrm{kpc}$, [279]).

${ }_{845}$ In the 400-800 $\mathrm{MHz}$ band the burst consisted of two narrow pulses separated by $29 \mathrm{~ms}$ and with a total fluence of $700 \mathrm{kJy} \mathrm{ms}$. Interestingly, two narrow pulses with the same separation, are visible also in the IBIS light curve and have a delay of $6.5 \mathrm{~ms}$ with respect to the radio ones. The discovery of simultaneous fast bursting emission at radio and high-energies from SGR 1935+2154 gives strong support 850 to models based on magnetars that have been proposed to explain the enigmatic class of sources known as fast radio bursts [289, 290].

\subsection{The 2004 giant flare from SGR 1806-20}

On December 27, 2004, a very bright burst was detected in the Anti-Coincidence Shield (ACS) of the SPI instrument and the corresponding light curve derived by the IBAS software was automatically published on-line in real time. Due to the large peak flux reached in the first $200 \mathrm{~ms}$ of the burst, the following tail was almost invisible on a linear scale, but, after a closer examination, its presence was noticed. The clear periodicity at $7.6 \mathrm{~s}$ visible in the burst tail unequivocally identified this event as a giant flare from SGR 1806-20 [291], similar to the two giant flares observed on 1979 March 5 from SGR 0525-66 and on 1998 August 27 from SGR 1900+14. This giant flare, first reported by INTEGRAL, was the culmination of two years of increasing bursting 


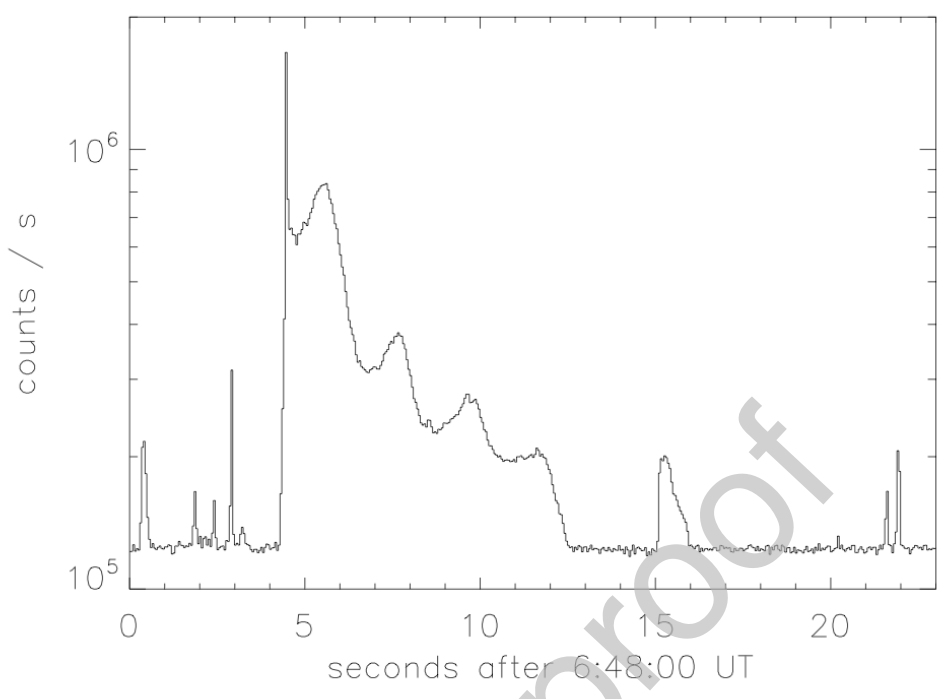

Figure 18: The SPI/ACS light curve at $E>80 \mathrm{keV}$ of a bright burst observed from 1E 1547.0-5408 on January 22,2009 . The tail following the bright initial pulse clearly show the NS rotation period of $2.1 \mathrm{~s}$. Figure from [284].

activity from SGR 1806-20, that was accompanied by a spectral hardening and an increase in the spin-down rate [292, 283].

Despite the downward revision in the source distance (from an initial estimate of $15 \mathrm{kpc}$, to the most likely value of $8.7 \mathrm{kpc}$ [293]) the SGR 1806-20 giant flare has been the most energetic observed so far, with a peak isotropic luminosity of $\sim 10^{47} \mathrm{erg} \mathrm{s}^{-1}$. The total energy release of $\sim 10^{46} \mathrm{erg}$ implies a catastrophic magnetic reconnection, associated to a major crustal fracture, and leading to a global reconfiguration of the NS's magnetic field.

870 Detailed results on the 2004 giant flare were obtained with different satellites [295, 296, 297]. In addition to the features reported in these works, thanks to the very large effective area of the ACS, INTEGRAL could discover a long-lasting emission that might be the first evidence for a soft $\gamma$-ray afterglow following a SGR giant flare [294]. As it can be seen in Fig. 19, after the end of the pulsating tail, the ACS count rate increased again, reaching a peak at $\mathrm{t} \sim 700 \mathrm{~s}$, and then returned to the pre-flare background level at $t \sim 3000-4000 \mathrm{~s}$. The presence of this long-lasting emission was later confirmed with Konus-Wind [298] and RHESSI [299] data, although with smaller statistics and on different time intervals.

The time evolution of the "afterglow" component seen by the INTEGRAL ACS above $\sim 80 \mathrm{keV}$ is well fitted by a power law decay with $F(t) \propto t^{-0.85}$. For a thermal Bremsstrahlung spectrum with temperature $k T=30 \mathrm{keV}$, the fluence at $E>80 \mathrm{keV}$ in the 400-4000 s time interval was $\sim 3 \times 10^{-4} \mathrm{erg} \mathrm{cm}^{-2}$, which is of the same order of that in the pulsating tail (i.e. in the 1-400 s time interval). However, since the ACS 


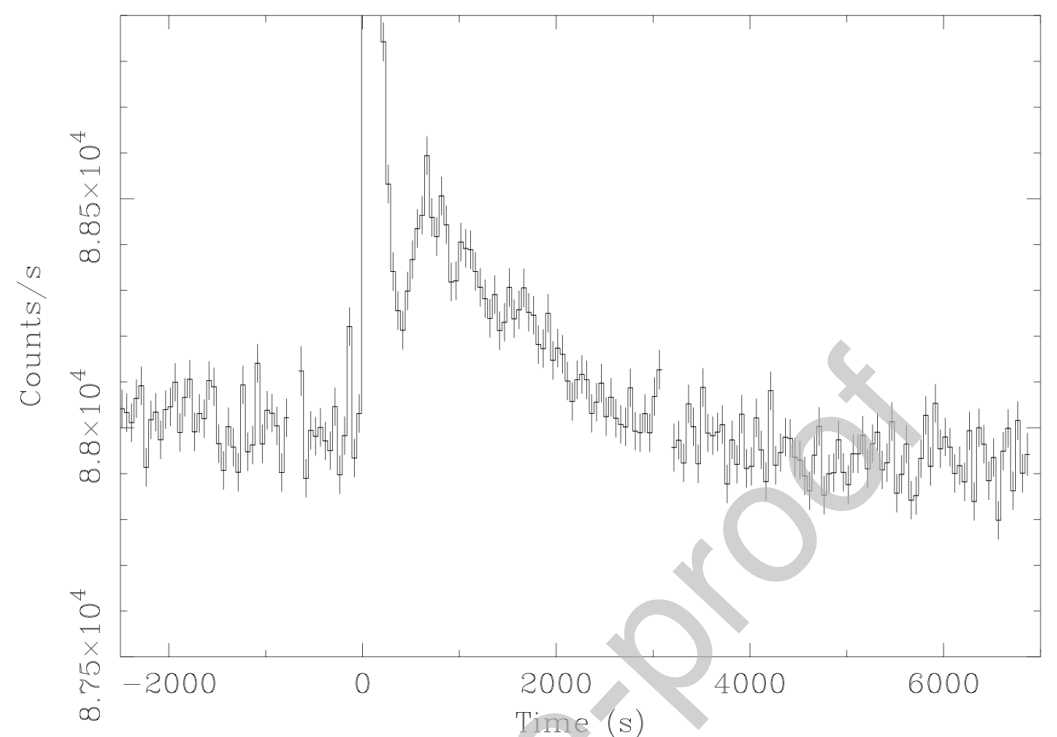

Figure 19: SPI/ACS light curve $(E>80 \mathrm{keV})$ of the December 27, 2004 giant flare from SGR 1806-20 (from [294]). Due to the rebinning at $50 \mathrm{~s}$, the $7.6 \mathrm{~s}$ pulsations in the tail time interval $(1-400 \mathrm{~s})$ are not visible in this figure. After the pulsating tail, the flux increases again, peaking $700 \mathrm{~s}$ after the beginning of the flare and decreasing below the background level about one hour later. Note that the peak of the flare at $\mathrm{t}=0$, reaching an observed count rate $>2 \times 10^{6}$ counts $\mathrm{s}^{-1}$, is out of the vertical scale.

does not provide spectral information, an estimate of the total energy, i.e. including the contribution at energies below $\sim 80 \mathrm{keV}$, is affected by a significant uncertainty due to the spectral extrapolation.

The power-law time evolution, as well as the hard power-law spectrum (photon in$\operatorname{dex} \Gamma \sim 1.6,[298]$ ), suggest to interpret this long-lasting emission as radiation caused by the interaction of relativistic ejecta from SGR 1806-20 with the circumstellar material [294], similar to the afterglows seen in $\gamma$-ray bursts. With standard models for $\gamma$-ray burst afterglows based on synchrotron emission, it is possible to relate the bulk Lorentz factor of the ejected material, $\gamma_{e j}$, with the time $t_{0}$ of the afterglow onset. The values observed with INTEGRAL give $\gamma_{e j} \sim 15\left(E / 5 \times 10^{43} \mathrm{erg}\right)^{1 / 8}\left(n / 0.1 \mathrm{~cm}^{-3}\right)^{-1 / 8}\left(t_{0} / 100 \mathrm{~s}\right)^{-3 / 8}$, where $n$ is the ambient density. $\gamma_{e j}$ is thus consistent with a mildly relativistic outflow, as also inferred from the analysis of the radio source that appeared after the giant flare [300]. 


\section{Future prospectives}

INTEGRAL has been successfully operated in Space for almost 18 years, and no major degradation of the instrument capabilities has been recorded so far. In principle, the mission scientific operations could continue until 2029, when the satellite is already planned to re-enter the Earth atmosphere. As emphasized multiple times in this review, the unique combination of sensitivity, timing resolution, large field of view and angular resolution of the INTEGRAL instruments has led to crucial advancements in our understanding of the pulsating hard X-ray sky.

For the study of the AMSPs and transitional millisecond X-ray pulsars in outburst, INTEGRAL will certainly continue to provide the means to discover additional rare members of these classes of sources. Any newly discovered object has provided unique insights into the accretion physics, leading to advancements in the understanding of the interaction between the accretion flow and the intense magnetic/gravitational field of explosions that often go off close to the compact object surface. So far, only one transitional X-ray pulsar has been caught during a bright X-ray outburst state, indeed thanks to INTEGRAL observations. It is thus of paramount importance to continue hunting for these peculiar objects in order to solve the unknown mechanisms driving the magnetars. The past 18 years of observations have proven that these objects are well at reach for the INTEGRAL instrumentation and we expect that additional outbursting events revealed during the INTEGRAL monitoring of the sky will produce rich data-sets to improve our understanding of the complex magnetospheric phenomenology that is driving the high energy emission from these systems.

The complementarity of the instruments on-board INTEGRAL with those of other last generation X-ray facilities, as XMM-Newton, Chandra, NuSTAR, and NICER, proved fundamental to go beyond the simple identification of new isolated and/or binary systems with rapidly rotating NSs. It also allowed to dig deeply inside the properties of their high energy emission, with INTEGRAL providing a unique contribution in the hardest X-ray energy band ( $\gtrsim 80 \mathrm{keV})$. Efforts are on-going to improve the rapidity of the response of the different facilities to the frequent INTEGRAL discoveries and to coordinate sub-sequent observations in a multi-messenger fashion [see, e.g., 301, and references therein].

Continued observations of the hard X-ray sky with INTEGRAL in the coming years will also certainly improve our sensitivity to detect fainter and fainter new hard sources. This will clearly boost the studies of these new discoveries with multi-wavelength emission, such as transitional millisecond pulsars. In this case, the INTEGRAL contribution will help us address fundamental questions such as which evolutionary channels produce transitional objects, how frequent is the occurrence of the faint disk state observed from these systems and ultimately which physical mechanism powers it and makes these objects different from standard AMSPs. Based on this and similar past experiences, we expect that the improved synergies between INTEGRAL and the other operating facilities in the multi-messenger context will help unveil the true nature of the newly discovered sources, providing further exciting discoveries and unexpected challenges in the decade to come. 


\section{Acknowledgements}

Al. P., D- d- M. and T. D. S- acknowledge financial support from ASI/INAF I/037/12/0, ASI/INAF 2017-14-H.0 (PI: De Rosa, PI: Belloni) and from INAF "Sostegno alla ricerca scientifica main streams dell'INAF", Presidential Decree 43/2018 and from "SKA/CTA projects", Presidential Decree N. 70/2016. J.P. was supported by the Ministry of Science and Higher Education of the Russian Federation grant 14.W03.31.0021. The INTEGRAL French teams acknowledge partial funding from the French Space Agency (CNES). Z.L. thanks the International Space Science Institute in Bern for the 950 hospitality. Z.L was supported by National Natural Science Foundation of China (Grant Nos. U1938107, 11703021, U1938107, 11873041). Ad. P. and S. M. acknowledge continuous support from the Italian Space Agency ASI through the ASI/INAF agreement n.2019-35-HH. D. F. T and the group at the Institute of Space Sciences acknowledges support via grants PGC2018-095512-B-I00, SGR2017-1383, andAYA2017-92402-

955 EXP. F .C. Z. is supported by a Juan de la Cierva fellowship. V. D. F. acknowledges Silesian University in Opava and Gruppo Nazionale di Fisica Matematica of Istituto Nazionale di Alta Matematica for support. This research was supported by the Polish National Science Centre grant number 2017/25/B/ST9/02805.

\section{References}

\section{References}

[1] A. Hewish, S. J. Bell, J. D. H. Pilkington, P. F. Scott, R. A. Collins, Observation of a Rapidly Pulsating Radio Source, Nat., 217 (5130) (1968) 709-713. doi : 10. $1038 / 217709$ a 0 .

[2] F. Pacini, Energy Emission from a Neutron Star, Nat., 216 (5115) (1967) 567568. doi : $10.1038 / 216567 a 0$.

[3] T. Gold, Rotating Neutron Stars as the Origin of the Pulsating Radio Sources, Nat., 218 (5143) (1968) 731-732. doi : 10.1038/218731a0.

[4] P. Ghosh, Rotation and Accretion Powered Pulsars, Vol. 10 of World Scientific Series in Astronomy and Astrophysics, World Scientific Publishing Co., 2007.

[5] C. Winkler, T. J. L. Courvoisier, G. Di Cocco, N. Gehrels, A. Giménez, S. Grebenev, W. Hermsen, J. M. Mas-Hesse, F. Lebrun, N. Lund, G. G. C. Palumbo, J. Paul, J. P. Roques, H. Schnopper, V. Schönfelder, R. Sunyaev, B. Teegarden, P. Ubertini, G. Vedrenne, A. J. Dean, The INTEGRAL mission, A\&A, 411 (2003) L1-L6. doi : 10 . 1051/0004-6361:20031288.

[6] P. Ubertini, F. Lebrun, G. Di Cocco, A. Bazzano, A. J. Bird, K. Broenstad, A. Goldwurm, G. La Rosa, C. Labanti, P. Laurent, I. F. Mirabel, E. M. Quadrini, B. Ramsey, V. Reglero, L. Sabau, B. Sacco, R. Staubert, L. Vigroux, M. C. Weisskopf, A. A. Zdziarski, IBIS: The Imager on-board INTEGRAL, A\&A, 411 (2003) L131-L139. doi : 10 . 1051/0004-6361:20031224. 
[7] F. Lebrun, J. P. Leray, P. Lavocat, J. Crétolle, M. Arquès, C. Blondel, C. Bonnin, A. Bouère, C. Cara, T. Chaleil, F. Daly, F. Desages, H. Dzitko, B. Horeau, P. Laurent, O. Limousin, F. Mathy, V. Mauguen, F. Meignier, F. Molinié, E. Poindron, M. Rouger, A. Sauvageon, T. Tourrette, ISGRI: The INTEGRAL Soft Gamma-Ray Imager, A\&A, 411 (2003) L141-L148. arXiv:astro-ph/ Q310362, doi:10.1051/0004-6361:20031367.

[8] L. Kuiper, W. Hermsen, R. Walter, L. Foschini, Absolute timing with IBIS, SPI and JEM-X aboard INTEGRAL. Crab main-pulse arrival times in radio, X-rays and high-energy gamma -rays, A\&A, 411 (2003) L31-L36. arXiv: astro-ph/0309178, doi : 10.1051/0004-6361:20031353.

[9] N. Lund, C. Budtz-Jørgensen, N. J. Westergaard, S. Brand t, I. L. Rasmussen, A. Hornstrup, C. A. Oxborrow, J. Chenevez, P. A. Jensen, S. Laursen, K. H. Andersen, P. B. Mogensen, I. Rasmussen, K. Omø, S. M. Pedersen, J. Polny, H. Andersson, T. Andersson, V. Kämäräinen, O. Vilhu, J. Huovelin, S. Maisala, M. Morawski, G. Juchnikowski, E. Costa, M. Feroci, A. Rubini, M. Rapisarda, E. Morelli, V. Carassiti, F. Frontera, C. Pelliciari, G. Loffredo, S. Martínez Núñez, V. Reglero, T. Velasco, S. Larsson, R. Svensson, A. A. Zdziarski, A. Castro-Tirado, P. Attina, M. Goria, G. Giulianelli, F. Cordero, M. Rezazad, M. Schmidt, R. Carli, C. Gomez, P. L. Jensen, G. Sarri, A. Tiemon, A. Orr, R. Much, P. Kretschmar, H. W. Schnopper, JEM-X: The X-ray monitor aboard INTEGRAL, A\&A, 411 (2003) L231-L238. doi:10.1051/0004-6361: 20031358.

[10] R. Wijnands, M. van der Klis, A millisecond pulsar in an X-ray binary system, Nat., 394 (6691) (1998) 344-346. doi : 10.1038/28557.

[11] A. Patruno, A. L. Watts, Accreting Millisecond X-Ray Pulsars, arXiv e-prints (2012) arXiv:1206.2727arXiv: 1206.2727.

[12] S. Campana, T. Di Salvo, Accreting Pulsars: Mixing-up Accretion Phases in Transitional Systems, in: L. Rezzolla, P. Pizzochero, D. I. Jones, N. Rea, I. Vidaña (Eds.), Astrophysics and Space Science Library, Vol. 457 of Astrophysics and Space Science Library, 2018, p. 149. arXiv: 1804.03422, doi: $10.1007 / 978-3-319-97616-7 \_4$.

[13] G. S. Bisnovatyi-Kogan, B. V. Komberg, Pulsars and close binary systems, SvA, 18 (1974) 217.

[14] M. A. Alpar, A. F. Cheng, M. A. Ruderman, J. Shaham, A new class of radio pulsars, Nat., 300 (5894) (1982) 728-730. doi : 10. 1038/300728a0.

[15] V. Radhakrishnan, G. Srinivasan, On the origin of the recently discovered ultrarapid pulsar, Current Science 51 (1982) 1096-1099.

[16] A. S. Fruchter, D. R. Stinebring, J. H. Taylor, A millisecond pulsar in an eclipsing binary, Nat., 333 (6170) (1988) 237-239. doi : 10.1038/333237a0. 
[17] P. Draghis, R. W. Romani, A. V. Filippenko, T. G. Brink, W. Zheng, J. P. Halpern, F. Camilo, Multiband Optical Light Curves of Black-widow Pulsars, ApJ, 883 (1) (2019) 108. arXiv:1908.00992, doi:10.3847/1538-4357/ ab378b.

[18] N. D’Amico, A. Possenti, R. N. Manchester, J. Sarkissian, A. G. Lyne, F. Camilo, An Eclipsing Millisecond Pulsar with a Possible Main-Sequence Companion in NGC 6397, ApJL, 561 (1) (2001) L89-L92. arXiv: astro-ph/ 0108250, doi : $10.1086 / 324562$.

[19] J. Strader, S. Swihart, L. Chomiuk, A. Bahramian, C. Britt, C. C. Cheung, K. Dage, J. Halpern, K.-L. Li, R. P. Mignani, J. A. Orosz, M. Peacock, R. Salinas, L. Shishkovsky, E. Tremou, Optical Spectroscopy and Demographics of Redback Millisecond Pulsar Binaries, ApJ, 872 (1) (2019) 42. arXiv: 1812 . 04626, doi : 10.3847/1538-4357/aafbaa.

[20] W. Kluzniak, M. Ruderman, J. Shaham, M. Tavani, Nature and evolution of the eclipsing millisecond binary pulsar PSR1957 + 20, Nat., 334 (6179) (1988) 225-227. doi : 10.1038/334225a0.

[21] E. P. J. van den Heuvel, J. van Paradijs, Fate of the companion stars of ultra-rapid pulsars, Nat., 334 (6179) (1988) 227-228. doi: 10 . 1038/334227a0.

[22] M. Ruderman, J. Shaham, M. Tavani, D. Eichler, Late Evolution of Very Low Mass X-Ray Binaries Sustained by Radiation from Their Primaries, ApJ, 343 (1989) 292. doi : 10.1086/167704.

[23] D. Chakrabarty, The spin distribution of millisecond X-ray pulsars, in: R. Wijnands, D. Altamirano, P. Soleri, N. Degenaar, N. Rea, P. Casella, A. Patruno, M. Linares (Eds.), American Institute of Physics Conference Series, Vol. 1068 of American Institute of Physics Conference Series, 2008, pp. 67-74. arXiv:0809.4031, doi : 10.1063/1.3031208.

[24] A. Papitto, D. F. Torres, N. Rea, T. M. Tauris, Spin frequency distributions of binary millisecond pulsars, A\&A, 566 (2014) A64. arXiv: 1403.6775, doi: 10. $1051 / 0004-6361 / 201321724$.

[25] A. Patruno, B. Haskell, N. Andersson, The Spin Distribution of Fast-spinning Neutron Stars in Low-mass X-Ray Binaries: Evidence for Two Subpopulations, ApJ, 850 (1) (2017) 106. arXiv: 1705.07669, doi : 10.3847/1538-4357/ aa927a.

[26] J. Poutanen, M. Gierliński, On the nature of the X-ray emission from the accreting millisecond pulsar SAX J1808.4-3658, MNRAS, 343 (4) (2003) 1301-1311. arXiv: astro-ph/0303084, doi : 10.1046/j . 1365-8711.2003.06773 .x.

[27] A. L. Watts, N. Andersson, D. Chakrabarty, M. Feroci, K. Hebeler, G. Israel, F. K. Lamb, M. C. Miller, S. Morsink, F. Özel, A. Patruno, J. Poutanen, D. Psaltis, A. Schwenk, A. W. Steiner, L. Stella, L. Tolos, M. van der 
Klis, Colloquium: Measuring the neutron star equation of state using x-ray timing, Reviews of Modern Physics 88 (2) (2016) 021001. arXiv: 1602 .01081, doi : 10.1103/RevModPhys.88.021001.

[28] S. E. Shaw, N. Mowlavi, J. Rodriguez, P. Ubertini, F. Capitanio, K. Ebisawa, D. Eckert, T. J. L. Courvoisier, N. Produit, R. Walter, M. Falanga, Discovery of the INTEGRAL X/ $\gamma$-ray transient IGR J00291+5934: A Comptonised accreting ms pulsar?, A\&A, 432 (1) (2005) L13-L16. arXiv: astro-ph/0501507, doi : 10. $1051 / 0004-6361: 200500011$.

[29] M. Falanga, L. Kuiper, J. Poutanen, E. W. Bonning, W. Hermsen, T. di Salvo, P. Goldoni, A. Goldwurm, S. E. Shaw, L. Stella, INTEGRAL and RXTE observations of accreting millisecond pulsar IGR J00291+5934 in outburst, A\&A, 444 (1) (2005) 15-24. arXiv:astro-ph/0508613, doi:10.1051/ 0004-6361:20053472.

[30] V. De Falco, L. Kuiper, E. Bozzo, D. K. Galloway, J. Poutanen, C. Ferrigno, L. Stella, M. Falanga, The 2015 outburst of the accretion-powered pulsar IGR J00291+5934: INTEGRAL and Swift observations, A\&A, 599 (2017) A88. arXiv: 1611.08218 , doi : 10.1051/0004-6361/201629575.

[31] M. Falanga, L. Kuiper, J. Poutanen, D. K. Galloway, E. W. Bonning, E. Bozzo, A. Goldwurm, W. Hermsen, L. Stella, Spectral and timing properties of the accreting X-ray millisecond pulsar IGR J17511-3057, A\&A, 529 (2011) A68. arXiv: 1012.0229, doi:10.1051/0004-6361/201016240.

[32] M. Falanga, L. Kuiper, J. Poutanen, D. K. Galloway, E. Bozzo, A. Goldwurm, W. Hermsen, L. Stella, Spectral and timing properties of the accreting X-ray millisecond pulsar IGR J17498-2921, A\&A, 545 (2012) A26. arXiv: 1208. 1384, doi : $10.1051 / 0004-6361 / 201219582$.

[33] P. Bordas, E. Kuulkers, J. Alfonso-Garzón, V. Beckmann, T. Bird, S. B. J. Chenevez, T. Courvoisier, M. Del Santo, A. Domingo, K. Ebisawa, C. Ferrigno, P. Jonker, P. Kretschmar, C. Markwardt, T. Oosterbroek, A. Paizis, K. Pottschmidt, C. Sánchez-Fernández, R. Wijnand s, A hard X-ray transient in the direction of Terzan 5 detected by INTEGRAL, The Astronomer's Telegram 2919 (2010) 1.

[34] C. Ferrigno, S. Brandt, E. Kuulkers, P. Bordas, E. Bozzo, J. Chenevez, C. Kouveliotou, A. J. van der Horst, INTEGRAL and RXTE spectral analysis of IGR J17480-2446, the new transient in Terzan 5., The Astronomer's Telegram 2940 (2010) 1 .

[35] T. E. Strohmayer, C. B. Markwardt, EXO 1745-248 is an $11 \mathrm{~Hz}$ Eclipsing Pulsar, The Astronomer's Telegram 2929 (2010) 1.

[36] A. Papitto, A. D’Aì, S. Motta, A. Riggio, L. Burderi, T. di Salvo, T. Belloni, R. Iaria, The spin and orbit of the newly discovered pulsar IGR J174802446, A\&A, 526 (2011) L3. arXiv: 1010 .4793, doi : 10.1051/0004-6361/ 201015974. 
[37] A. Papitto, C. Ferrigno, E. Bozzo, N. Rea, L. Pavan, L. Burderi, M. Burgay, S. Campana, T. di Salvo, M. Falanga, M. D. Filipović, P. C. C. Freire, J. W. T. Hessels, A. Possenti, S. M. Ransom, A. Riggio, P. Romano, J. M. Sarkissian, I. H. Stairs, L. Stella, D. F. Torres, M. H. Wieringa, G. F. Wong, Swings between rotation and accretion power in a binary millisecond pulsar, Nat., 501 (7468) (2013) 517-520. arXiv: 1305.3884, doi : 10.1038/nature12470.

[38] C. Ferrigno, E. Bozzo, A. Papitto, N. Rea, L. Pavan, S. Campana, M. Wieringa, M. Filipović, M. Falanga, L. Stella, Hiccup accretion in the swinging pulsar IGR J18245-2452, A\&A, 567 (2014) A77. arXiv: 1310. 7784, doi : 10. 1051/ 0004-6361/201322904.

[39] V. De Falco, L. Kuiper, E. Bozzo, C. Ferrigno, J. Poutanen, L. Stella, M. Falanga, The transitional millisecond pulsar IGR J18245-2452 during its 2013 outburst at X-rays and soft gamma-rays, A\&A, 603 (2017) A16. arXiv: 1704.04181, doi : 10.1051/0004-6361/201730600.

[40] T. Strohmayer, L. Keek, IGR J17062-6143 Is an Accreting Millisecond XRay Pulsar, ApJL, 836 (2) (2017) L23. arXiv: 1702 . 05449, doi : 10. 3847/ 2041-8213/aa5e51.

[41] A. Sanna, A. Bahramian, E. Bozzo, C. Heinke, D. Altamirano, R. Wijnands, N. Degenaar, T. Maccarone, A. Riggio, T. Di Salvo, R. Iaria, M. Burgay, A. Possenti, C. Ferrigno, A. Papitto, G. R. Sivakoff, N. D'Amico, L. Burderi, Discovery of $105 \mathrm{~Hz}$ coherent pulsations in the ultracompact binary IGR J165973704, A\&A, 610 (2018) L2. arXiv: 1711.03092, doi : 10 . 1051/0004-6361/ 201732262 .

[42] A. Sanna, E. Bozzo, A. Papitto, A. Riggio, C. Ferrigno, T. Di Salvo, R. Iaria, S. M. Mazzola, N. D Amico, L. Burderi, XMM-Newton detection of the $2.1 \mathrm{~ms}$ coherent pulsations from IGR J17379-3747, A\&A, 616 (2018) L17. arXiv: 1807.08574, doi : $10.1051 / 0004-6361 / 201833205$.

[43] T. E. Strohmayer, P. S. Ray, K. C. Gendreau, P. M. Bult, S. Guillot, S. Mahmoodifar, G. K. Jaisawal, Z. Arzoumanian, D. Altamirano, S. Bogdanov, D. Chakrabarty, T. Enoto, C. B. Markwardt, F. Ozel, S. M. Ransom, NICER discovers millisecond pulsations from the neutron star LMXB IGR J17379-3747, The Astronomer's Telegram 11507 (2018) 1.

[44] A. Sanna, C. Ferrigno, P. S. Ray, L. Ducci, G. K. Jaisawal, T. Enoto, E. Bozzo, D. Altamirano, T. Di Salvo, T. E. Strohmayer, A. Papitto, A. Riggio, L. Burderi, P. M. Bult, S. Bogdanov, A. F. Gambino, A. Marino, R. Iaria, Z. Arzoumanian, D. Chakrabarty, K. C. Gendreau, S. Guillot, C. Markwardt, M. T. Wolff, NuSTAR and NICER reveal IGR J17591-2342 as a new accreting millisecond X-ray pulsar, A\&A, 617 (2018) L8. arXiv: 1808.10195, doi: 10. $1051 / 0004-6361 / 201834160$. 
[45] M. Del Santo, E. Bozzo, E. Kuulkers, A. Bazzano, V. Beckmann, T. Bird, A. Bodaghee, J. Chenevez, A. Domingo, P. Jonker, P. Kretschmar, C. Markwardt, A. Paizis, K. Pottschmidt, C. Sanchez-Fernandez, R. Wijnands, INTEGRAL observations of SAX J1808.4-3658 currently in outburst, The Astronomer's Telegram 7380 (2015) 1.

[46] A. Patruno, A. Jaodand, L. Kuiper, P. Bult, J. W. T. Hessels, C. Knigge, A. R. King, R. Wijnands, M. van der Klis, Radio Pulse Search and X-Ray Monitoring of SAX J1808.4-3658: What Causes Its Orbital Evolution?, ApJ, 841 (2) (2017) 98. arXiv: 1611.06023 , doi : 10.3847/1538-4357/aa6f5b.

[47] C. Ferrigno, V. Savchenko, J. Chenevez, J. Wilms, E. Kuulkers, Galactic Bulge Monitoring Team, E. Bozzo, L. Ducci, INTEGRAL observations of the Galactic Center region: Sgr A* and SAX J1808.4-3658., The Astronomer's Telegram 13035 (2019) 1.

[48] S. A. Grebenev, S. V. Molkov, R. A. Sunyaev, An outburst of the accreting millisecond X-ray pulsar XTE J1751-305 detected with INTEGRAL, The Astronomer's Telegram 446 (2005) 1.

[49] M. Falanga, S. Soldi, S. Shaw, A. Goldwurm, G. Belanger, D. Porquet, F. Melia, R. Terrier, F. Yusef-Zadeh, INTEGRAL IBIS/ISGRI hard X-ray detection of the accreting millisecond pulsar XTE J1751-305, The Astronomer's Telegram 1046 (2007) 1 .

[50] J. Chenevez, E. Kuulkers, V. Beckmann, A. Bird, S. Brandt, A. Domingo, K. Ebisawa, P. Jonker, P. Kretschmar, C. Markwardt, T. Oosterbroek, A. Paizis, D. Risquez, C. Sanchez-Fernandez, S. Shaw, R. Wijnands, INTEGRAL sees transient activity in the Galactic Bulge: XTE J1751-305 and GRS 1741.9-2853 in outburst, The Astronomer's Telegram 2235 (2009) 1.

[51] M. Falanga, J. M. Bonnet-Bidaud, J. Poutanen, R. Farinelli, A. Martocchia, P. Goldoni, J. L. Qu, L. Kuiper, A. Goldwurm, INTEGRAL spectroscopy of the accreting millisecond pulsar XTE J1807-294 in outburst, A\&A, 436 (2) (2005) 647-652. arXiv:astro-ph/0503292, doi:10.1051/0004-6361: 20042575 .

[52] M. Falanga, J. Poutanen, E. W. Bonning, L. Kuiper, J. M. Bonnet-Bidaud, A. Goldwurm, W. Hermsen, L. Stella, Simultaneous INTEGRAL and RXTE observations of the accreting millisecond pulsar HETE J1900.1-2455, A\&A, 464 (3) (2007) 1069-1074. arXiv:astro-ph/0609776, doi:10.1051/ 0004-6361:20066457.

[53] E. Kuulkers, E. Bozzo, A. Bazzano, V. Beckmann, T. Bird, A. Bodaghee, J. Chenevez, M. Del Santo, A. Domingo, P. Jonker, P. Kretschmar, A. Paizis, K. Pottschmidt, C. Markwardt, C. Sanchez-Fernandez, R. Wijnands, INTEGRAL detection of a hard X-ray transient in NGC 6440, The Astronomer's Telegram 7098 (2015) 1. 
[54] Z. Li, V. De Falco, M. Falanga, E. Bozzo, L. Kuiper, J. Poutanen, A. Cumming, D. K. Galloway, S. Zhang, Mixed H/He bursts in SAX J1748.9-2021 during the spectral change of its 2015 outburst, A\&A, 620 (2018) A114. arXiv: 1810. Q5490, doi : $10.1051 / 0004-6361 / 201833857$.

[55] L. Di Gesu, E. Bozzo, E. Kuulkers, A. Bazzano, V. Beckmann, T. Bird, A. Bodaghee, J. Chenevez, M. Del Santo, A. Domingo, P. Jonker, P. Kretschmar, C. Markwardt, A. Paizis, K. Pottschmidt, C. Sánchez-Fernández, R. Wijnand s, INTEGRAL detection of the on-going outburst from NGC 6440 and a new outburst likely from GRS 1747-312 in Terzan 6., The Astronomer's Telegram 10832 (2017) 1 .

[56] L. Pavan, J. Chenevez, E. Bozzo, E. Kuulkers, J. Alfonso-Garzon, V. Beckmann, T. Bird, S. Brand t, T. Courvoisier, A. Domingo, K. Ebisawa, P. Jonker, P. Kretschmar, C. Markwardt, T. Oosterbroek, A. Paizis, C. Sanchez-Fernandez, R. Wijnands, INTEGRAL and Swift detection of high energy emission from Swift J1749.4-2807, The Astronomer's Telegram 2548 (2010) 1.

[57] J. Chenevez, S. Brandt, C. Sanchez-Fernandez, E. Kuulkers, J. AlfonsoGarzón, V. Beckmann, T. Bird, T. Courvoisier, A. Domingo, K. Ebisawa, P. Jonker, P. Kretschmar, C. Markwardt, T. Oosterbroek, A. Paizis, R. Wijnands, INTEGRAL/JEM-X detection of an X-ray burst from Swift J1749.4-2807, The Astronomer's Telegram 2561 (2010) 1.

[58] C. Ferrigno, E. Bozzo, M. Falanga, L. Stella, S. Campana, T. Belloni, G. L. Israel, L. Pavan, E. Kuulkers, A. Papitto, INTEGRAL, Swift, and RXTE observations of the $518 \mathrm{~Hz}$ accreting transient pulsar Swift J1749.4-2807, A\&A, 525 (2011) A48. arXiv: 1005 .4554, doi : 10.1051/0004-6361/201015033.

[59] A. Sanna, A. Papitto, L. Burderi, E. Bozzo, A. Riggio, T. Di Salvo, C. Ferrigno, N. Rea, R. Iaria, Discovery of a new accreting millisecond X-ray pulsar in the globular cluster NGC 2808, A\&A, 598 (2017) A34. arXiv: 1611. 02995, doi : 10. $1051 / 0004-6361 / 201629406$.

[60] E. Bozzo, C. Ferrigno, A. Papitto, A. Sanna, L. Burderi, N. Rea, T. Di Salvo, INTEGRAL observation of MAXI J0911-655, The Astronomer's Telegram 8986 (2016) 1 .

[61] L. Ducci, K. Watanabe, C. Sanchez, R. Diehl, E. Bozzo, C. Ferrigno, INTEGRAL detection of MAXI J0911-655: still active, The Astronomer's Telegram 9738 (2016) 1.

[62] M. Falanga, L. Kuiper, J. Poutanen, D. K. Galloway, E. Bozzo, A. Goldwurm, W. Hermsen, L. Stella, Accreting millisecond X-ray pulsars: 10 years of INTEGRAL observations, arXiv e-prints (2013) arXiv:1302.2843arXiv: 1302.2843. 
[63] E. Kuulkers, S. Shaw, A. Paizis, N. Mowlavi, T. Courvoisier, K. Ebisawa, P. Kretschmar, C. Markwardt, T. Oosterbroek, A. Orr, R. Wijnands, Announcement of INTEGRAL Galactic Bulge monitoring program and (re)brightening of GRO J1655-40, The Astronomer's Telegram 438 (2005) 1.

[64] E. Kuulkers, S. E. Shaw, A. Paizis, J. Chenevez, S. Brandt, T. J. L. Courvoisier, A. Domingo, K. Ebisawa, P. Kretschmar, C. B. Markwardt, N. Mowlavi, T. Oosterbroek, A. Orr, D. Rísquez, C. Sanchez-Fernandez, R. Wijnands, The INTEGRAL Galactic bulge monitoring program: the first 1.5 years, A\&A, 466 (2) (2007) 595-618. arXiv:astro-ph/0701244, doi : 10.1051/ 0004-6361:20066651.

[65] D. Altamirano, Y. Cavecchi, A. Patruno, A. Watts, M. Linares, N. Degenaar, M. Kalamkar, M. van der Klis, N. Rea, P. Casella, M. Armas Padilla, R. Kaur, Y. J. Yang, P. Soleri, R. Wijnands, Discovery of an Accreting Millisecond Pulsar in the Eclipsing Binary System SWIFT J1749.4-2807, ApJL, 727 (1) (2011) L18. arXiv: 1005 . 3527, doi : $10.1088 / 2041-8205 / 727 / 1 /$ L18.

[66] P. G. Jonker, M. A. P. Torres, D. Steeghs, D. Chakrabarty, Chandra X-ray and Gemini near-infrared observations of the eclipsing millisecond pulsar SWIFT J1749.4-2807 in quiescence, MNRAS, 429 (1) (2013) 523-528. arXiv: 1211. 1156, doi : 10.1093/mnras/sts363.

[67] J. G. Victor, E. Kuulkers, L. Sidoli, C. Sanchez-Fernand ez, K. Watanabe, L. Pavan, E. Bozzo, INTEGRAL detection of continued hard X-ray emission from MAXI J0911-655, The Astronomer's Telegram 10425 (2017) 1.

[68] A. R. King, H. Ritter, The light curves of soft X-ray transients, MNRAS, 293 (1) (1998) L42-L48. doi : 10 . 1046/j . 1365-8711.1998.01295 .x.

[69] H. V. Bradt, R. E. Rothschild, J. H. Swank, X-ray timing explorer mission, A\&AS, 97 (1993) 355-360.

[70] C. R. Powell, C. A. Haswell, M. Falanga, Mass transfer during low-mass Xray transient decays, MNRAS, 374 (2) (2007) 466-476. arXiv:astro-ph/ 0610108, doi : 10.1111/j.1365-2966.2006.11144.x.

[71] F. Lewis, D. M. Russell, P. G. Jonker, M. Linares, V. Tudose, P. Roche, J. S. Clark, M. A. P. Torres, D. Maitra, C. G. Bassa, D. Steeghs, A. Patruno, S. Migliari, R. Wijnands, G. Nelemans, L. J. Kewley, V. E. Stroud, M. Modjaz, J. S. Bloom, C. H. Blake, D. Starr, The double-peaked 2008 outburst of the accreting milli-second X-ray pulsar, IGR J00291+5934, A\&A, 517 (2010) A72. arXiv: 1005 . 1178, doi : 10.1051/0004-6361/201014382.

[72] J. M. Hartman, D. K. Galloway, D. Chakrabarty, A Double Outburst from IGR J00291+5934: Implications for Accretion Disk Instability Theory, ApJ, 726 (1) (2011) 26. arXiv: 1006. 1908, doi : 10.1088/0004-637X/726/1/26. 
[73] A. Patruno, D. Maitra, P. A. Curran, C. D'Angelo, J. K. Fridriksson, D. M. Russell, M. Middleton, R. Wijnand s, The Reflares and Outburst Evolution in the Accreting Millisecond Pulsar SAX J1808.4-3658: A Disk Truncated Near Co-Rotation?, ApJ, 817 (2) (2016) 100. arXiv: 1504.05048, doi : 10.3847/ 0004-637X/817/2/100.

[74] P. Bult, D. Chakrabarty, Z. Arzoumanian, K. C. Gendreau, S. Guillot, C. Malacaria, P. S. Ray, T. E. Strohmayer, Timing the pulsations of the accreting millisecond pulsar SAX J1808.4-3658 during its 2019 outburst, arXiv e-prints (2019) arXiv:1910.03062arXiv: 1910.03062.

[75] J. Poutanen, Accretion-powered millisecond pulsars, Advances in Space Research 38 (12) (2006) 2697-2703. arXiv:astro-ph/0510038, doi:10. $1016 /$ j .asr. 2006.04 .025 .

[76] M. Gierliński, C. Done, D. Barret, Phase-resolved X-ray spectroscopy of the millisecond pulsar SAX J1808.4-3658, MNRAS, 331 (1) (2002) 141-153. arXiv: astro-ph/0111310, doi : 10.1046/j . 1365-8711.2002.05174.x.

[77] M. Gierliński, J. Poutanen, Physics of accretion in the millisecond pulsar XTE J1751-305, MNRAS, 359 (4) (2005) 1261-1276. arXiv: astro-ph/0411716, doi : 10.1111/j.1365-2966.2005.09004.x.

[78] J. Poutanen, R. Svensson, The Two-Phase Pair Corona Model for Active Galactic Nuclei and X-Ray Binaries: How to Obtain Exact Solutions, ApJ, 470 (1996) 249. arXiv: astro-ph/9605073, doi : 10.1086/177865.

[79] P. Casella, D. Altamirano, A. Patruno, R. Wijnands, M. van der Klis, Discovery of Coherent Millisecond X-Ray Pulsations in Aquila X-1, ApJL, 674 (1) (2008) L41. arXiv: 0708.1110, doi:10.1086/528982.

[80] J. Rodriguez, S. E. Shaw, S. Corbel, The faint 2005 hard state outburst of Aquila X-1 seen by INTEGRAL and RXTE, A\&A, 451 (3) (2006) 1045-1048. arXiv: astro-ph/0602235, doi : 10.1051/0004-6361:20054412.

[81] F. Jansen, D. Lumb, B. Altieri, J. Clavel, M. Ehle, C. Erd, C. Gabriel, M. Guainazzi, P. Gondoin, R. Much, R. Munoz, M. Santos, N. Schartel, D. Texier, G. Vacanti, XMM-Newton observatory. I. The spacecraft and operations, A\&A, 365 (2001) L1-L6. doi : 10. 1051/0004-6361:20000036.

[82] F. A. Harrison, W. W. Craig, F. E. Christensen, C. J. Hailey, W. W. Zhang, S. E. Boggs, D. Stern, W. R. Cook, K. Forster, P. Giommi, B. W. Grefenstette, Y. Kim, T. Kitaguchi, J. E. Koglin, K. K. Madsen, P. H. Mao, H. Miyasaka, K. Mori, M. Perri, M. J. Pivovaroff, S. Puccetti, V. R. Rana, N. J. Westergaard, J. Willis, A. Zoglauer, H. An, M. Bachetti, N. M. Barrière, E. C. Bellm, V. Bhalerao, N. F. Brejnholt, F. Fuerst, C. C. Liebe, C. B. Markwardt, M. Nynka, J. K. Vogel, D. J. Walton, D. R. Wik, D. M. Alexander, L. R. Cominsky, A. E. Hornschemeier, A. Hornstrup, V. M. Kaspi, G. M. Madejski, G. Matt, S. Molendi, D. M. Smith, J. A. Tomsick, M. Ajello, D. R. Ballantyne, M. Baloković, D. Barret, F. E. Bauer, 
R. D. Blandford, W. N. Brandt, L. W. Brenneman, J. Chiang, D. Chakrabarty, J. Chenevez, A. Comastri, F. Dufour, M. Elvis, A. C. Fabian, D. Farrah, C. L. Fryer, E. V. Gotthelf, J. E. Grindlay, D. J. Helfand, R. Krivonos, D. L. Meier, J. M. Miller, L. Natalucci, P. Ogle, E. O. Ofek, A. Ptak, S. P. Reynolds, J. R. Rigby, G. Tagliaferri, S. E. Thorsett, E. Treister, C. M. Urry, The Nuclear Spectroscopic Telescope Array (NuSTAR) High-energy X-Ray Mission, ApJ, 770 (2) (2013) 103. arXiv: 1301.7307, doi : 10.1088/0004-637X/770/2/103.

[83] A. Papitto, T. Di Salvo, A. D’Aì, R. Iaria, L. Burderi, A. Riggio, M. T. Menna, N. R. Robba, XMM-Newton detects a relativistically broadened iron line in the spectrum of the ms X-ray pulsar SAX J1808.4-3658, A\&A, 493 (3) (2009) L39L43. arXiv:0812 .1149, doi:10.1051/0004-6361:200811401.

[84] E. M. Cackett, D. Altamirano, A. Patruno, J. M. Miller, M. Reynolds, M. Linares, R. Wijnands, Broad Relativistic Iron Emission Line Observed in SAX J1808.4-3658, ApJL, 694 (1) (2009) L21-L25. arXiv: \$901.3142, doi : $10.1088 / 0004-637 \mathrm{X} / 694 / 1 / \mathrm{L} 21$.

[85] A. Sanna, F. Pintore, E. Bozzo, C. Ferrigno, A. Papitto, A. Riggio, T. Di Salvo, R. Iaria, A. D'Aì, E. Egron, L. Burderi, Spectral and timing properties of IGR J00291+5934 during its 2015 outburst, MNRAS, 466 (3) (2017) 2910-2917. arXiv: 1612.03865 , doi : $10.1093 / \mathrm{mnras} / \mathrm{stw} 3332$.

[86] T. Di Salvo, A. Sanna, L. Burderi, A. Papitto, R. Iaria, A. F. Gambino, A. Riggio, NuSTAR and XMM-Newton broad-band spectrum of SAX J1808.4-3658 during its latest outburst in 2015, MNRAS, 483 (1) (2019) 767-779. arXiv: 1811. Q0940, doi : $10.1093 / \mathrm{mnras} / \mathrm{sty} 2974$.

[87] M. A. Nowak, A. Paizis, G. K. Jaisawal, J. Chenevez, S. Chaty, F. Fortin, J. Rodriguez, J. Wilms, Chandra-HETGS Characterization of an Outflowing Wind in the Accreting Millisecond Pulsar IGR J17591-2342, ApJ, 874 (1) (2019) 69. arXiv:1902.09577, doi : 10.3847/1538-4357/ab0a71.

[88] A. Paizis, M. A. Nowak, J. Wilms, T. J. L. Courvoisier, K. Ebisawa, J. Rodriguez, P. Ubertini, Chandra and RXTE spectroscopy of the accreting msec pulsar IGR J00291+5934, A\&A, 444 (2) (2005) 357-363. arXiv:astro-ph/ Q508258, doi : 10.1051/0004-6361:20053419.

[89] A. Patruno, D. Altamirano, J. W. T. Hessels, P. Casella, R. Wijnands, M. van der Klis, Phase-Coherent Timing of the Accreting Millisecond Pulsar SAX J1748.92021, ApJ, 690 (2) (2009) 1856-1865. arXiv:0801.1031, doi:10.1088/ $0004-637 \mathrm{X} / 690 / 2 / 1856$.

[90] G. Hasinger, M. van der Klis, Two patterns of correlated X-ray timing and spectral behaviour in low-mass X-ray binaries., A\&A, 225 (1989) 79-96.

[91] F. Pintore, A. Sanna, T. Di Salvo, M. Del Santo, A. Riggio, A. D’Aì, L. Burderi, F. Scarano, R. Iaria, Broad-band spectral analysis of the accreting millisecond X-ray pulsar SAX J1748.9-2021, MNRAS, 457 (3) (2016) 2988-2998. arXiv: 1601.05215, doi : 10.1093/mnras/stw176. 
[92] T. Di Salvo, P. Goldoni, L. Stella, M. van der Klis, A. Bazzano, L. Burderi, R. Farinelli, F. Frontera, G. L. Israel, M. Méndez, I. F. Mirabel, N. R. Robba, P. Sizun, P. Ubertini, W. H. G. Lewin, A Hard X-Ray View of Scorpius X1 with INTEGRAL: Nonthermal Emission?, ApJL, 649 (2) (2006) L91-L94. arXiv: astro-ph/0608335, doi : 10.1086/508489.

[93] M. G. Revnivtsev, S. S. Tsygankov, E. M. Churazov, R. A. Krivonos, Hard Xray emission of Sco X-1, MNRAS, 445 (2) (2014) 1205-1212. arXiv: 1409. 1679, doi : $10.1093 / \mathrm{mnras} /$ stu1831.

[94] A. Paizis, R. Farinelli, L. Titarchuk, T. J. L. Courvoisier, A. Bazzano, V. Beckmann, F. Frontera, P. Goldoni, E. Kuulkers, S. Mereghetti, J. Rodriguez, O. Vilhu, Average hard X-ray emission from NS LMXBs: observational evidence of different spectral states in NS LMXBs, A\&A, 459 (1) (2006) 187-197. arXiv: astro-ph/0607592, doi : 10 . 1051/0004-6361:20065792.

[95] F. Pintore, T. Di Salvo, E. Bozzo, A. Sanna, L. Burderi, A. D’Aì, A. Riggio, F. Scarano, R. Iaria, Study of the reflection spectrum of the accreting neutron star GX 3+1 using XMM-Newton and INTEGRAL, MNRAS, 450 (2) (2015) 2016-2024. arXiv: 1504.00684, doi : 10.1093/mnras/stv758.

[96] F. Pintore, A. Sanna, A. Riggio, T. Di Salvo, S. Mereghetti, E. Bozzo, C. Sánchez-Fernández, L. Burderi, R. Iaria, A faint outburst of the accreting millisecond X-ray pulsar SAX J1748.9-2021 in NGC 6440, MNRAS, 479 (3) (2018) 4084-4090. arXiv:1806.10944, doi : 10.1093/mnras/sty1735.

[97] Z. Arzoumanian, K. C. Gendreau, C. L. Baker, T. Cazeau, P. Hestnes, J. W. Kellogg, S. J. Kenyon, R. P. Kozon, K. C. Liu, S. S. Manthripragada, C. B. Markwardt, A. L. Mitchell, J. W. Mitchell, C. A. Monroe, T. Okajima, S. E. Pollard, D. F. Powers, B. J. Savadkin, L. B. Winternitz, P. T. Chen, M. R. Wright, R. Foster, G. Prigozhin, R. Remillard, J. Doty, The neutron star interior composition explorer (NICER): mission definition, in: Proc. of the SPIE, , Vol. 9144 of Society of Photo-Optical Instrumentation Engineers (SPIE) Conference Series, 2014, p. 914420. doi : 10.1117/12.2056811.

[98] L. Kuiper, M. Falanga, V. De Falco, W. Hermsen, E. Bozzo, C. Ferrigno, INTEGRAL ToO observation of IGR J00291+5934 during its July/August 2015 outburst: spin and orbital ephemerides update for the July/August 2015 epoch, The Astronomer's Telegram 7949 (2015) 1.

[99] M. Falanga, L. Titarchuk, Energy-dependent $~ 100 \mu$ s Time Lags as Observational Evidence of Comptonization Effects in the Neutron Star Plasma Environment, ApJ, 661 (2) (2007) 1084-1088. arXiv:astro-ph/0702453, doi : $10.1086 / 514805$

[100] A. Sanna, L. Burderi, A. Riggio, F. Pintore, T. Di Salvo, A. F. Gambino, R. Iaria, M. Matranga, F. Scarano, Timing of the accreting millisecond pulsar SAX J1748.9-2021 during its 2015 outburst, MNRAS, 459 (2) (2016) 1340-1349. arXiv: 1603.08757 , doi : 10.1093/mnras/stw740. 
[101] A. Patruno, D. Altamirano, C. Messenger, The long-term evolution of the accreting millisecond X-ray pulsar SwiftJ1756.9-2508, MNRAS, 403 (3) (2010) 1426-1432. arXiv:0910.2920, doi : 10.1111/j .1365-2966.2010.16202.

[102] A. Sanna, F. Pintore, A. Riggio, S. M. Mazzola, E. Bozzo, T. Di Salvo, C. Ferrigno, A. F. Gambino, A. Papitto, R. Iaria, L. Burderi, SWIFT J1756.9-2508: spectral and timing properties of its 2018 outburst, MNRAS, 481 (2) (2018) 1658-1666. arXiv: 1808.06796, doi : 10.1093/mnras/sty2316.

[103] W. Cui, E. H. Morgan, L. G. Titarchuk, Soft Phase Lags of Pulsed Emission from the Millisecond X-Ray Pulsar SAX J1808.4-3658, ApJL, 504 (1) (1998) L27-L30. arXiv: astro-ph/9807081, doi : 10. 1086/311569.

[104] L. Titarchuk, W. Cui, K. Wood, Why Is It Difficult to Detect a Millisecond Pulsar in Neutron Star X-Ray Binaries?, ApJL, 576 (1) (2002) L49-L52. arXiv: astro-ph/0207552, doi:10.1086/343099.

[105] A. Ibragimov, J. Poutanen, Accreting millisecond pulsar SAX J1808.4-3658 during its 2002 outburst: evidence for a receding disc, MNRAS, 400 (1) (2009) 492-508. arXiv:0901.0073, doi : 10.1111/j .1365-2966.2009.15477.x.

[106] W. H. G. Lewin, J. van Paradijs, R. E. Taam, X-Ray Bursts, Space Sci. Rev., 62 (3-4) (1993) 223-389. doi : 10.1007/BF00196124.

[107] D. K. Galloway, L. Keek, Thermonuclear X-ray bursts, arXiv e-prints (2017) arXiv:1712.06227arXiv: 1712.06227

[108] I. V. Chelovekov, S. A. Grebenev, I. A. Mereminskiy, A. V. Prosvetov, Type I X-ray Bursts Detected by the JEM-X Telescope Onboard the INTEGRAL Observatory in 2003-2015, Astronomy Letters 43 (12) (2017) 781-795. doi : $10.1134 /$ S1063773717120076.

[109] A. Papitto, T. Di Salvo, L. Burderi, T. M. Belloni, L. Stella, E. Bozzo, A. D’Aì, C. Ferrigno, R. Iaria, S. Motta, A. Riggio, A. Tramacere, The pulse profile and spin evolution of the accreting pulsar in Terzan 5, IGR J17480-2446, during its 2010 outburst, MNRAS, 423 (2) (2012) 1178-1193. arXiv: 1203.4096, doi: $10.1111 / \mathrm{j} .1365-2966.2012 .20945 . \mathrm{x}$.

[110] V. Testa, T. di Salvo, F. D’Antona, M. T. Menna, P. Ventura, L. Burderi, A. Riggio, R. Iaria, A. D'Aì, A. Papitto, N. Robba, The near-IR counterpart of IGR J17480-2446 in Terzan 5, A\&A, 547 (2012) A28. arXiv: 1210.8261, doi : $10.1051 / 0004-6361 / 201219904$.

[111] A. Patruno, M. A. Alpar, M. van der Klis, E. P. J. van den Heuvel, The Peculiar Evolutionary History of IGR J17480-2446 in Terzan 5, ApJ, 752 (1) (2012) 33. arXiv: 1112 .5315, doi : 10.1088/0004-637X/752/1/33. 
[112] S. Motta, A. D’Aì, A. Papitto, A. Riggio, T. di Salvo, L. Burderi, T. Belloni, L. Stella, R. Iaria, X-ray bursts and burst oscillations from the slowly spinning X-ray pulsar IGR J17480-2446 (Terzan 5), MNRAS, 414 (2) (2011) 1508-1516. arXiv: 1102.1368, doi:10.1111/j.1365-2966.2011.18483.x.

[113] M. Linares, D. Altamirano, D. Chakrabarty, A. Cumming, L. Keek, Millihertz Quasi-periodic Oscillations and Thermonuclear Bursts from Terzan 5: A Showcase of Burning Regimes, ApJ, 748 (2) (2012) 82. arXiv: 1111.3978, doi : $10.1088 / 0004-637 \mathrm{X} / 748 / 2 / 82$.

[114] D. Altamirano, A. Ingram, M. van der Klis, R. Wijnand s, M. Linares, J. Homan, Low-frequency Quasi-periodic Oscillation from the $11 \mathrm{~Hz}$ Accreting Pulsar in Terzan 5: Not Frame Dragging, ApJL, 759 (1) (2012) L20. arXiv: 1210 . 1494, doi : $10.1088 / 2041-8205 / 759 / 1 /$ L20.

[115] M. Revnivtsev, E. Churazov, M. Gilfanov, R. Sunyaev, New class of low frequency QPOs: Signature of nuclear burning or accretion disk instabilities?, A\&A, 372 (2001) 138-144. arXiv:astro-ph/0011110, doi:10.1051/ $0004-6361: 20010434$.

[116] M. Linares, D. Chakrabarty, M. van der Klis, On the Cooling Tails of Thermonuclear X-ray Bursts: The IGR J17480-2446 Link, ApJL, 733 (2) (2011) L17. arXiv: 1102 . 1455, doi : 10.1088/2041-8205/733/2/L17.

[117] M. Chakraborty, S. Bhattacharyya, A. Mukherjee, Terzan 5 transient IGR J17480-2446: variation of burst and spectral properties with spectral states, MNRAS, 418 (1) (2011) 490-499. arXiv: 1102.1033, doi:10.1111/j . 1365-2966.2011.19499. $\mathrm{x}$

[118] A. Heger, A. Cumming, S. E. Woosley, Millihertz Quasi-periodic Oscillations from Marginally Stable Nuclear Burning on an Accreting Neutron Star, ApJ, 665 (2) (2007) 1311-1320. arXiv: astro-ph/0511292, doi : 10. 1086/ 517491.

[119] Y. Cavecchi, A. Patruno, B. Haskell, A. L. Watts, Y. Levin, M. Linares, D. Altamirano, R. Wijnands, M. van der Klis, Implications of Burst Oscillations from the Slowly Rotating Accreting Pulsar IGR J17480-2446 in the Globular Cluster Terzan 5, ApJL, 740 (1) (2011) L8. arXiv: 1102.1548, doi : 10. $1088 / 2041-8205 / 740 / 1 / \mathrm{L} 8$.

[120] J. M. Miller, D. Maitra, E. M. Cackett, S. Bhattacharyya, T. E. Strohmayer, A Fast X-ray Disk Wind in the Transient Pulsar IGR J17480-2446 in Terzan 5, ApJL, 731 (1) (2011) L7. arXiv: 1101 .2377, doi : 10. 1088/2041-8205/ 731/1/L7.

[121] N. Degenaar, E. F. Brown, R. Wijnands, Evidence for crust cooling in the transiently accreting $11-\mathrm{Hz} \mathrm{X}$-ray pulsar in the globular cluster Terzan $5, \mathrm{MN}$ RAS, 418 (1) (2011) L152-L156. arXiv:1107.5317, doi:10.1111/j. 1745-3933.2011.01164.x. 
[122] N. Degenaar, R. Wijnands, E. F. Brown, D. Altamirano, E. M. Cackett, J. Fridriksson, J. Homan, C. O. Heinke, J. M. Miller, D. Pooley, G. R. Sivakoff, Continued Neutron Star Crust Cooling of the $11 \mathrm{~Hz}$ X-Ray Pulsar in Terzan 5: A Challenge to Heating and Cooling Models?, ApJ, 775 (1) (2013) 48. arXiv: 1306.2345 , doi : $10.1088 / 0004-637 \mathrm{X} / 775 / 1 / 48$.

[123] L. S. Ootes, S. Vats, D. Page, R. Wijnands, A. S. Parikh, N. Degenaar, M. J. P. Wijngaarden, D. Altamirano, A. Bahramian, E. M. Cackett, C. O. Heinke, J. Homan, J. M. Miller, Continued cooling of the accretion-heated neutron star crust in the X-ray transient IGR J17480-2446 located in the globular cluster Terzan 5, MNRAS, 487 (1) (2019) 1447-1461. arXiv:1805.00610, doi: 10. $1093 / \mathrm{mnras} / \mathrm{stz} 1406$.

[124] N. Degenaar, D. R. Ballantyne, T. Belloni, M. Chakraborty, Y.-P. Chen, L. Ji, P. Kretschmar, E. Kuulkers, J. Li, T. J. Maccarone, J. Malzac, S. Zhang, S.-N. Zhang, Accretion Disks and Coronae in the X-Ray Flashlight, Space Sci. Rev., 214 (1) (2018) 15. arXiv: 1711.06272, doi: 10.1007/ s11214-017-0448-3.

[125] J. J. E. Kajava, C. Sánchez-Fernández, E. Kuulkers, J. Poutanen, X-ray burstinduced spectral variability in 4U 1728-34, A\&A, 599 (2017) A89. arXiv: 1611.03976, doi : 10.1051/0004-6361/201629542.

[126] L. Ji, S. Zhang, Y. P. Chen, S. N. Zhang, P. Kretschmar, J. M. Wang, J. Li, Possible hard X-ray shortages in bursts from KS 1731-260 and 4U 1705-44, A\&A, 564 (2014) A20. arXiv: 1402 . 3802, doi : 10 . 1051/0004-6361/201322981.

[127] L. Stella, S. Campana, M. Colpi, S. Mereghetti, M. Tavani, Do Quiescent Soft X-Ray Transients Contain Millisecond Radio Pulsars?, ApJL, 423 (1994) L47. doi : $10.1086 / 187232$.

[128] S. Campana, M. Colpi, S. Mereghetti, L. Stella, M. Tavani, The neutron stars of Soft X-ray Transients, A\&AR, 8 (4) (1998) 279-316. arXiv:astro-ph/ 9805079, doi : $10.1007 / \mathrm{s} 001590050012$.

[129] L. Burderi, A. Possenti, F. D’Antona, T. Di Salvo, M. Burgay, L. Stella, M. T. Menna, R. Iaria, S. Campana, N. d'Amico, Where May Ultrafast Rotating Neutron Stars Be Hidden?, ApJL, 560 (1) (2001) L71-L74. arXiv: astro-ph/0109088, doi : $10.1086 / 324220$.

[130] S. Campana, L. Stella, F. Gastaldello, S. Mereghetti, M. Colpi, G. L. Israel, L. Burderi, T. Di Salvo, R. N. Robba, An XMM-Newton Study of the $401 \mathrm{~Hz}$ Accreting Pulsar SAX J1808.4-3658 in Quiescence, ApJL, 575 (1) (2002) L15L19. arXiv: astro-ph/0206376, doi : 10.1086/342505.

[131] L. Burderi, T. Di Salvo, F. D’Antona, N. R. Robba, V. Testa, The optical counterpart to SAX J1808.4-3658 in quiescence: Evidence of an active radio pulsar?, A\&A, 404 (2003) L43-L46. arXiv:astro-ph/0305157, doi : 10. $1051 / 0004-6361: 20030669$. 
[132] J. M. Hartman, A. Patruno, D. Chakrabarty, D. L. Kaplan, C. B. Markwardt, E. H. Morgan, P. S. Ray, M. van der Klis, R. Wijnands, The Long-Term Evolution of the Spin, Pulse Shape, and Orbit of the Accretion-powered Millisecond Pulsar SAX J1808.4-3658, ApJ, 675 (2) (2008) 1468-1486. arXiv: 0708.0211, doi : 10.1086/527461.

[133] T. di Salvo, L. Burderi, A. Riggio, A. Papitto, M. T. Menna, Orbital evolution of an accreting millisecond pulsar: witnessing the banquet of a hidden black widow?, MNRAS, 389 (4) (2008) 1851-1857. arXiv:0708.0498, doi:10. $1111 / \mathrm{j} .1365-2966.2008 .13709 . \mathrm{x}$.

[134] M. S. E. Roberts, Surrounded by spiders! New black widows and redbacks in the Galactic field, in: J. van Leeuwen (Ed.), Neutron Stars and Pulsars: Challenges and Opportunities after 80 years, Vol. 291 of IAU Symposium, 2013, pp. $127-$ 132. arXiv: 1210.6903 , doi : 10.1017/S174392131202337X.

[135] M. Burgay, L. Burderi, A. Possenti, N. D’Amico, R. N. Manchester, A. G. Lyne, F. Camilo, S. Campana, A Search for Pulsars in Quiescent Soft X-Ray Transients. I., ApJ, 589 (2) (2003) 902-910. arXiv:astro-ph/0302128, doi : $10.1086 / 374690$.

[136] M. N. Iacolina, M. Burgay, L. Burderi, A. Possenti, T. di Salvo, Search for pulsations at high radio frequencies from accreting millisecond X-ray pulsars in quiescence, A\&A, 519 (2010) A13. arXiv: 1006.4260, doi:10.1051/ $0004-6361 / 201014025$.

[137] A. M. Archibald, I. H. Stairs, S. M. Ransom, V. M. Kaspi, V. I. Kondratiev, D. R. Lorimer, M. A. McLaughlin, J. Boyles, J. W. T. Hessels, R. Lynch, J. van Leeuwen, M. S. E. Roberts, F. Jenet, D. J. Champion, R. Rosen, B. N. Barlow, B. H. Dunlap, R. A. Remillard, A Radio Pulsar/X-ray Binary Link, Science 324 (5933) (2009) 1411. arXiv:0905 . 3397, doi : 10.1126/science. 1172740 .

[138] D. Eckert, M. Del Santo, A. Bazzano, K. Watanabe, A. Paizis, E. Bozzo, C. Ferrigno, I. Caballero, L. Sidoli, L. Kuiper, IGR J18245-2452: a new hard X-ray transient discovered by INTEGRAL, The Astronomer's Telegram 4925 (2013) 1.

[139] J. Strader, K.-L. Li, L. Chomiuk, C. O. Heinke, A. Udalski, M. Peacock, L. Shishkovsky, E. Tremou, A New $\gamma$-Ray Loud, Eclipsing Low-mass XRay Binary, ApJ, 831 (1) (2016) 89. arXiv:1608.02583, doi:10.3847/ 0004-637X/831/1/89.

[140] F. Coti Zelati, A. Papitto, D. de Martino, D. A. H. Buckley, A. Odendaal, J. Li, T. D. Russell, D. F. Torres, S. M. Mazzola, E. Bozzo, M. Gromadzki, S. Campana, N. Rea, C. Ferrigno, S. Migliari, Prolonged sub-luminous state of the new transitional pulsar candidate CXOU J110926.4-650224, A\&A, 622 (2019) A211. arXiv: 1903.04526, doi : 10.1051/0004-6361/201834835. 
[141] C. G. Bassa, A. Patruno, J. W. T. Hessels, E. F. Keane, B. Monard, E. K. Mahony, S. Bogdanov, S. Corbel, P. G. Edwards, A. M. Archibald, G. H. Janssen, B. W. Stappers, S. Tendulkar, A state change in the low-mass X-ray binary XSS J12270-4859, MNRAS, 441 (2) (2014) 1825-1830. arXiv: 1402.0765, doi : $10.1093 / \mathrm{mnras} / \mathrm{stu} 708$.

[142] D. de Martino, M. Falanga, J. M. Bonnet-Bidaud, T. Belloni, M. Mouchet, N. Masetti, I. Andruchow, S. A. Cellone, K. Mukai, G. Matt, The intriguing nature of the high-energy gamma ray source XSS J12270-4859, A\&A, 515 (2010) A25. arXiv: 1002 . 3740, doi : 10.1051/0004-6361/200913802.

[143] M. Linares, X-Ray States of Redback Millisecond Pulsars, ApJ, 795 (1) (2014) 72. arXiv: 1406.2384 , doi : 10.1088/0004-637X/795/1/72.

[146] A. T. Deller, J. Moldon, J. C. A. Miller-Jones, A. Patruno, J. W. T. Hessels, A. M. Archibald, Z. Paragi, G. Heald, N. Vilchez, Radio Imaging Observations of PSR J1023+0038 in an LMXB State, ApJ, 809 (1) (2015) 13. arXiv: 1412 . 5155, doi : $10.1088 / 0004-637 \mathrm{X} / 809 / 1 / 13$.

[147] S. Bogdanov, A. T. Deller, J. C. A. Miller-Jones, A. M. Archibald, J. W. T. Hessels, A. Jaodand, A. Patruno, C. Bassa, C. D’Angelo, Simultaneous Chandra and VLA Observations of the Transitional Millisecond Pulsar PSR J1023+0038: Anti-correlated X-Ray and Radio Variability, ApJ, 856 (1) (2018) 54. arXiv: 1709.08574, doi : 10.3847/1538-4357/aaaeb9.

[148] B. W. Stappers, A. M. Archibald, J. W. T. Hessels, C. G. Bassa, S. Bogdanov, G. H. Janssen, V. M. Kaspi, A. G. Lyne, A. Patruno, S. Tendulkar, A. B. Hill, T. Glanzman, A State Change in the Missing Link Binary Pulsar System PSR J1023+0038, ApJ, 790 (1) (2014) 39. arXiv: 1311.7506, doi : $10.1088 / 0004-637 \mathrm{X} / 790 / 1 / 39$.

[149] D. F. Torres, L. Ji, J. Li, A. r. Papitto, N. Rea, E. de Oña Wilhelmi, S. Zhang, A Search for Transitions between States in Redbacks and Black Widows Using Seven Years of Fermi-LAT Observations, ApJ, 836 (1) (2017) 68. arXiv: 1612 . 07083, doi : 10.3847/1538-4357/836/1/68. 
[150] A. M. Archibald, S. Bogdanov, A. Patruno, J. W. T. Hessels, A. T. Deller, C. Bassa, G. H. Janssen, V. M. Kaspi, A. G. Lyne, B. W. Stappers, S. P. Tendulkar, C. R. D'Angelo, R. Wijnands, Accretion-powered Pulsations in an Apparently Quiescent Neutron Star Binary, ApJ, 807 (1) (2015) 62. arXiv: 1412.1306, doi : 10.1088/0004-637X/807/1/62.

[151] A. Papitto, D. de Martino, T. M. Belloni, M. Burgay, A. Pellizzoni, A. Possenti, D. F. Torres, X-ray coherent pulsations during a sub-luminous accretion disc state of the transitional millisecond pulsar XSS J12270-4859., MNRAS, 449 (2015) L26-L30. arXiv: 1412 . 4252, doi : 10 . 1093/mnrasl/slv013.

[152] F. Ambrosino, A. Papitto, L. Stella, F. Meddi, P. Cretaro, L. Burderi, T. Di Salvo, G. L. Israel, A. Ghedina, L. Di Fabrizio, L. Riverol, Optical pulsations from a transitional millisecond pulsar, Nature Astronomy 1 (2017) 854-858. arXiv: 1709.01946, doi : 10.1038/s41550-017-0266-2.

[153] A. Papitto, F. Ambrosino, L. Stella, D. Torres, F. Coti Zelati, A. Ghedina, F. Meddi, A. Sanna, P. Casella, Y. Dallilar, S. Eikenberry, G. L. Israel, F. Onori, S. Piranomonte, E. Bozzo, L. Burderi, S. Campana, D. de Martino, T. Di Salvo, C. Ferrigno, N. Rea, A. Riggio, S. Serrano, A. Veledina, L. Zampieri, Pulsating in Unison at Optical and X-Ray Energies: Simultaneous High Time Resolution Observations of the Transitional Millisecond Pulsar PSR J1023+0038, ApJ, 882 (2) (2019) 104. arXiv: 1904, 10433, doi : 10. 3847/1538-4357/ab2fdf.

[154] N. Masetti, L. Morelli, E. Palazzi, G. Galaz, L. Bassani, A. Bazzano, A. J. Bird, A. J. Dean, G. L. Israel, R. Landi, A. Malizia, D. Minniti, F. Schiavone, J. B. Stephen, P. Ubertini, R. Walter, Unveiling the nature of INTEGRAL objects through optical spectroscopy. V. Identification and properties of 21 southern hard X-ray sources, A\&A, 459 (1) (2006) 21-30. arXiv: astro-ph/0608394, doi : 10.1051/0004-6361:20066055.

[155] A. B. Hill, A. Szostek, S. Corbel, F. Camilo, R. H. D. Corbet, R. Dubois, G. Dubus, P. G. Edwards, E. C. Ferrara, M. Kerr, E. Koerding, D. Kozieł, Ł. Stawarz, The bright unidentified $\gamma$-ray source 1FGL J1227.9-4852: can it be associated with a low-mass X-ray binary?, MNRAS, 415 (1) (2011) 235-243. arXiv: 1103.2637 , doi : 10.1111/j .1365-2966.2011.18692.x.

[156] D. de Martino, T. Belloni, M. Falanga, A. Papitto, S. Motta, A. Pellizzoni, Y. Evangelista, G. Piano, N. Masetti, J. M. Bonnet-Bidaud, M. Mouchet, K. Mukai, A. Possenti, X-ray follow-ups of XSS J12270-4859: a low-mass X-ray binary with gamma-ray Fermi-LAT association, A\&A, 550 (2013) A89. arXiv: 1212 . 1615, doi : 10.1051/0004-6361/201220393.

[157] A. Papitto, D. F. Torres, J. Li, A propeller scenario for the gamma-ray emission of low-mass X-ray binaries: the case of XSS J12270-4859, MNRAS, 438 (3) (2014) 2105-2116. arXiv: 1312 .0456, doi : 10.1093/mnras/stt2336.

[158] A. Papitto, D. F. Torres, A Propeller Model for the Sub-luminous State of the Transitional Millisecond Pulsar PSR J1023+0038, ApJ, 807 (1) (2015) 33. arXiv: 1504.05029 , doi : $10.1088 / 0004-637 \mathrm{X} / 807 / 1 / 33$. 
[159] C. R. D'Angelo, H. C. Spruit, Accretion discs trapped near corotation, MNRAS, 420 (1) (2012) 416-429. arXiv: 1108 . 3833, doi:10.1111/j.1365-2966.

$2011.20046 . x$.

[160] E. Bozzo, S. Ascenzi, L. Ducci, A. Papitto, L. Burderi, L. Stella, Magnetospheric radius of an inclined rotator in the magnetically threaded disk model, A\&A, 617 (2018) A126. arXiv: 1806. 11516, doi : 10. 1051/0004-6361/201732004.

[161] J. Roy, P. S. Ray, B. Bhattacharyya, B. Stappers, J. N. Chengalur, J. Deneva, F. Camilo, T. J. Johnson, M. Wolff, J. W. T. Hessels, C. G. Bassa, E. F. Keane, E. C. Ferrara, A. K. Harding, K. S. Wood, Discovery of Psr J1227-4853: A Transition from a Low-mass X-Ray Binary to a Redback Millisecond Pulsar, ApJL, 800 (1) (2015) L12. arXiv: 1412.4735, doi : 10.1088/2041-8205/ $800 / 1 /$ L12.

[162] The Fermi-LAT collaboration, Fermi Large Area Telescope Fourth Source Catalog, arXiv e-prints (2019) arXiv:1902.10045arXiv: 1902.10045.

[163] D. J. Thompson, A. K. Harding, W. Hermsen, M. P. Ulmer, Gamma-ray pulsars: the Compton Observatory contribution to the study of isolated neutron stars, in: C. D. Dermer, M. S. Strickman, J. D. Kurfess (Eds.), Proceedings of the Fourth Compton Symposium, Vol. 410 of American Institute of Physics Conference Series, 1997, pp. 39-56. doi : 10.1063/1.54038.

[164] L. Kuiper, W. Hermsen, J. M. Krijger, K. Bennett, A. Carramiñana, V. Schönfelder, M. Bailes, R. N. Manchester, COMPTEL detection of pulsed gamma -ray emission from PSR B1509-58 up to at least $10 \mathrm{MeV}, \mathrm{A} \& \mathrm{~A}, 351$ (1999) 119-132. arXiv:astro-ph/9903474.

[165] W. Hermsen, L. Kuiper, R. Diehl, G. Lichti, V. Schoenfelder, A. W. Strong, A. Connors, J. Ryan, K. Bennett, M. Busetta, A. Carramiñana, R. Buccheri, I. A. Grenier, Gamma-Ray Pulsar Studies with COMPTEL, ApJS, 92 (1994) 559. doi: $10.1086 / 192016$.

[166] J. K. Daugherty, A. K. Harding, Polar CAP Models of Gamma-Ray Pulsars: Emission from Single Poles of Nearly Aligned Rotators, ApJ, 429 (1994) 325. doi : $10.1086 / 174321$

[167] K. S. Cheng, C. Ho, M. Ruderman, Energetic Radiation from Rapidly Spinning Pulsars. I. Outer Magnetosphere Gaps, ApJ, 300 (1986) 500. doi:10.1086/ 163829.

[168] K. S. Cheng, C. Ho, M. Ruderman, Energetic Radiation from Rapidly Spinning Pulsars. II. VELA and Crab, ApJ, 300 (1986) 522. doi : 10. 1086/163830.

[169] G. Srinivasan, Millisecond pulsars: A new population of gamma ray sources?, Advances in Space Research 10 (2) (1990) 167-178. doi:10.1016/ Q273-1177 (90)90137-0. 
[170] L. Kuiper, W. Hermsen, F. Verbunt, D. J. Thompson, I. H. Stairs, A. G. Lyne, M. S. Strickman, G. Cusumano, The likely detection of pulsed high-energy gamma -ray emission from millisecond pulsar PSR J0218+4232, A\&A, 359 (2000) 615-626. arXiv: astro-ph/0005338.

[171] A. K. Harding, V. V. Usov, A. G. Muslimov, High-Energy Emission from Millisecond Pulsars, ApJ, 622 (1) (2005) 531-543. arXiv: astro-ph/0411805, doi : $10.1086 / 427840$.

[172] J. Dyks, B. Rudak, Two-Pole Caustic Model for High-Energy Light Curves of Pulsars, ApJ, 598 (2) (2003) 1201-1206. arXiv:astro-ph/0303006, doi: $10.1086 / 379052$.

[173] A. G. Muslimov, A. K. Harding, High-Altitude Particle Acceleration and Radiation in Pulsar Slot Gaps, ApJ, 606 (2) (2004) 1143-1153. arXiv: astro-ph/ 0402462, doi : $10.1086 / 383079$.

[174] A. Y. Chen, A. M. Beloborodov, Electrodynamics of Axisymmetric Pulsar Magnetosphere with Electron-Positron Discharge: A Numerical Experiment, ApJL, 795 (1) (2014) L22. arXiv: 1406.7834 , doi : 10 . 1088/2041-8205/795/1/ L22.

[175] I. Mochol, J. Petri, Very high energy emission as a probe of relativistic magnetic reconnection in pulsar winds., MNRAS, 449 (2015) L51-L55. arXiv: 1501. 07123, doi : $10.1093 / \mathrm{mnrasl} / \mathrm{slv}$ 018.

[176] B. Cerutti, A. A. Philippov, A. Spitkovsky, Modelling high-energy pulsar light curves from first principles, MNRAS, 457 (3) (2016) 2401-2414. arXiv: 1511. 01785, doi : 10,1093/mnras/stw124.

[177] A. A. Philippov, A. Spitkovsky, Ab-initio Pulsar Magnetosphere: Particle Acceleration in Oblique Rotators and High-energy Emission Modeling, ApJ, 855 (2) (2018) 94. arXiv: 1707 . 04323, doi : 10 . 3847/1538-4357/aaabbc.

1680 [178] G. Brambilla, C. Kalapotharakos, A. N. Timokhin, A. K. Harding, D. Kazanas, Electron-Positron Pair Flow and Current Composition in the Pulsar Magnetosphere, ApJ, 858 (2) (2018) 81. arXiv:1710.03536, doi:10.3847/ 1538-4357/aab3e1.

[179] C. Kalapotharakos, G. Brambilla, A. Timokhin, A. K. Harding, D. Kazanas, Three-dimensional Kinetic Pulsar Magnetosphere Models: Connecting to Gamma-Ray Observations, ApJ, 857 (1) (2018) 44. arXiv: 1710.03170, doi : 10.3847/1538-4357/aab550.

[180] G. Cusumano, T. Mineo, E. Massaro, L. Nicastro, E. Trussoni, S. Massaglia, W. Hermsen, L. Kuiper, The curved X-ray spectrum of PSR B1509-58 observed with BeppoSAX, A\&A, 375 (2001) 397-404. doi:10.1051/0004-6361: 20010884. 
[181] J. de Plaa, L. Kuiper, W. Hermsen, Hard X-ray timing and spectral properties of PSR B0540-69, A\&A, 400 (2003) 1013-1019. arXiv: astro-ph/0301195, doi : 10.1051/0004-6361:20030039.

[182] A. H. Rots, K. Jahoda, D. J. Macomb, N. Kawai, Y. Saito, V. M. Kaspi, A. G. Lyne, R. N. Manchester, D. C. Backer, A. L. Somer, D. Marsden, R. E. Rothschild, Rossi X-Ray Timing Explorer Absolute Timing Results for the Pulsars B1821-24 and B1509-58, ApJ, 501 (2) (1998) 749-757. arXiv:astro-ph/ 9801251, doi : $10.1086 / 305836$.

[183] L. Kuiper, W. Hermsen, B. Stappers, Chandra and RXTE studies of the X-ray $/ \gamma$ ray millisecond pulsar PSR J0218+4232, Advances in Space Research 33 (4) (2004) 507-512. arXiv:astro-ph/0306622, doi:10.1016/j .asr . 2003. 08.019.

[184] G. Cusumano, W. Hermsen, M. Kramer, L. Kuiper, O. Löhmer, E. Massaro, T. Mineo, L. Nicastro, B. W. Stappers, The phase of the radio and X-ray pulses of PSR B1937+21, A\&A, 410 (2003) L9-L12. arXiv: astro-ph/0309580, doi : 10.1051/0004-6361:20031368.

[185] S. J. Sturner, M. Forot, P. Laurent, Observations of PSR B1509-58 using INTEGRAL Core Program Data, in: V. Schoenfelder, G. Lichti, C. Winkler (Eds.), 5th INTEGRAL Workshop on the INTEGRAL Universe, Vol. 552 of ESA Special Publication, 2004, p. 479

[186] M. Forot, W. Hermsen, M. Renaud, P. Laurent, I. Grenier, P. Goret, B. Khelifi, L. Kuiper, High-Energy Particles in the Wind Nebula of Pulsar B1509-58 as Seen by INTEGRAL, ApJL, 651 (1) (2006) L45-L48. arXiv:astro-ph/ 0609663, doi : 10.1086/509077.

[187] D. Götz, S. Mereghetti, D. Merlini, L. Sidoli, T. Belloni, An INTEGRAL hard X-ray survey of the Large Magellanic Cloud, A\&A, 448 (3) (2006) 873-880. arXiv:astro-ph/0510770, doi : 10.1051/0004-6361:20053744.

[188] A. Słowikowska, G. Kanbach, A. Stefanescu, Fully Resolved Optical Polarization of the Crab Pulsar, in: S. Ritz, P. Michelson, C. A. Meegan (Eds.), The First GLAST Symposium, Vol. 921 of American Institute of Physics Conference Series, 2007, pp. 419-420. doi : 10.1063/1.2757381.

[189] R. Landi, A. de Rosa, A. J. Dean, L. Bassani, P. Ubertini, A. J. Bird, HESS J1616-508: likely to be powered by PSR J1617-5055, MNRAS, 380 (3) (2007) 926-932. arXiv:0707.0832, doi : 10.1111/j.1365-2966.2007.12168.x.

[190] A. J. Dean, A. de Rosa, V. A. McBride, R. Landi, A. B. Hill, L. Bassani, A. Bazzano, A. J. Bird, P. Ubertini, INTEGRAL observations of PSR J18111925 and its associated pulsar wind nebula, MNRAS, 384 (1) (2008) L29-L33. arXiv:0711.0648, doi : 10.1111/j .1745-3933.2007.00415.x. 
[199] A. K. Harding, C. Kalapotharakos, Multiwavelength Polarization of Rotationpowered Pulsars, ApJ, 840 (2) (2017) 73. arXiv: 1704 . 06183, doi : 10.3847/ 1538-4357/aa6ead.

[200] J. McDonald, P. O’Connor, D. de Burca, A. Golden, A. Shearer, Inverse mapping of polarized optical emission from pulsars: basic formulation and determination of emission altitude, MNRAS, 417 (1) (2011) 730-744. arXiv: 1106.5207, doi : 10.1111/j.1365-2966.2011.19318.x.

[201] M. C. Weisskopf, E. H. Silver, H. L. Kestenbaum, K. S. Long, R. Novick, A precision measurement of the X-ray polarization of the Crab Nebula without pulsar contamination., ApJL, 220 (1978) L117-L121. doi : 10. 1086/182648. 
[202] A. J. Dean, D. J. Clark, J. B. Stephen, V. A. McBride, L. Bassani, A. Bazzano, A. J. Bird, A. B. Hill, S. E. Shaw, P. Ubertini, Polarized Gamma-Ray Emission from the Crab, Science 321 (5893) (2008) 1183. doi : 10.1126/science. 1149056.

[203] M. Forot, P. Laurent, I. A. Grenier, C. Gouiffès, F. Lebrun, Polarization of the Crab Pulsar and Nebula as Observed by the INTEGRAL/IBIS Telescope, ApJL, 688 (1) (2008) L29. arXiv:0809. 1292, doi : 10. 1086/593974.

[204] F. G. Smith, D. H. P. Jones, J. S. B. Dick, C. D. Pike, The optical polarization of the Crab pulsar., MNRAS, 233 (1988) 305-319. doi : 10.1093/mnras/233. 2.305 .

[205] A. Słowikowska, G. Kanbach, M. Kramer, A. Stefanescu, Optical polarization of the Crab pulsar: precision measurements and comparison to the radio emission, MNRAS, 397 (1) (2009) 103-123. arXiv:0901.4559, doi: $10.1111 / \mathrm{j} .1365-2966.2009 .14935 . \mathrm{x}$.

[206] P. Moran, A. Shearer, C. Gouiffès, P. Laurent, INTEGRAL/IBIS and optical observations of the Crab nebula/pulsar polarisation, in: Proceedings of "An INTEGRAL view of the high-energy sky (the first 10 years)" - 9th INTEGRAL Workshop and celebration of the 10th anniversary of the launch (INTEGRAL 2012). 15-19 October 2012. Bibliotheque Nationale de France, 2012, p. 8.

[207] P. Moran, A. Shearer, R. P. Mignani, A. Słowikowska, A. De Luca, C. Gouiffès, P. Laurent, Optical polarimetry of the inner Crab nebula and pulsar, MNRAS, 433 (3) (2013) 2564-2575. arXiv: 1305.6824, doi:10.1093/mnras/ stt931.

[208] P. Moran, G. Kyne, C. Gouiffès, P. Laurent, G. Hallinan, R. M. Redfern, A. Shearer, A recent change in the optical and $\gamma$-ray polarization of the Crab nebula and pulsar, MNRAS, 456 (3) (2016) 2974-2981. arXiv: 1511.07641, doi $: 10.1093 / \mathrm{mnras} / \mathrm{stv} 2780$.

[209] A. Słowikowska, R. Mignani, G. Kanbach, K. Krzeszowski, Decomposition of the Optical Polarisation Components of the Crab Pulsar and its Nebula, Vol. 466 of Astronomical Society of the Pacific Conference Series, 2012, p. 37.

[210] J. J. Hester, P. A. Scowen, R. Sankrit, C. J. Burrows, I. Gallagher, John S., J. A. Holtzman, A. Watson, J. T. Trauger, G. E. Ballester, S. Casertano, J. T. Clarke, D. Crisp, R. W. Evans, R. E. Griffiths, J. G. Hoessel, J. Krist, R. Lynds, J. R. Mould, J. O’Neil, Earl J., K. R. Stapelfeldt, J. A. Westphal, WFPC2 Studies of the Crab Nebula. I. HST and ROSAT Imaging of the Synchrotron Nebula, ApJ, 448 (1995) 240. doi : 10.1086/175956.

[211] M. F. Bietenholz, D. A. Frail, J. J. Hester, The Crab Nebula's Moving Wisps in Radio, ApJ, 560 (1) (2001) 254-260. arXiv:astro-ph/0106339, doi: $10.1086 / 322244$. 
[212] J. D. Scargle, Activity in the Crab Nebula, ApJ, 156 (1969) 401. doi : 10. 1086/ 149978.

[213] M. G. Kirsch, U. G. Briel, D. Burrows, S. Campana, G. Cusumano, K. Ebisawa, M. J. Freyberg, M. Guainazzi, F. Haberl, K. Jahoda, J. Kaastra, P. Kretschmar, S. Larsson, P. Lubiński, K. Mori, P. Plucinsky, A. M. Pollock, R. Rothschild, S. Sembay, J. Wilms, M. Yamamoto, Crab: the standard X-ray candle with all (modern) x-ray satellites, in: O. H. W. Siegmund (Ed.), Proc. of the SPIE, , Vol. 5898 of Society of Photo-Optical Instrumentation Engineers (SPIE) Conference Series, 2005, pp. 22-33. arXiv : astro-ph/0508235, doi : $10.1117 / 12.616893$.

[214] M. C. Weisskopf, M. Guainazzi, K. Jahoda, N. Shaposhnikov, S. L. O’Dell, V. E. Zavlin, C. Wilson-Hodge, R. F. Elsner, On Calibrations Using the Crab Nebula and Models of the Nebular X-Ray Emission, ApJ, 713 (2) (2010) 912919. arXiv: 1003 . 1916, doi : 10.1088/0004-637X/713/2/912.

[215] A. A. Abdo, M. Ackermann, M. Ajello, A. Allafort, L. Baldini, J. Ballet, G. Barbiellini, D. Bastieri, K. Bechtol, R. Bellazzini, B. Berenji, R. D. Blandford, E. D. Bloom, E. Bonamente, A. W. Borgland, A. Bouvier, T. J. Brand t, J. Bregeon, A. Brez, M. Brigida, P. Bruel, R. Buehler, S. Buson, G. A. Caliandro, R. A. Cameron, A. Cannon, P. A. Caraveo, J. M. Casand jian, Ö. Çelik, E. Charles, A. Chekhtman, C. C. Cheung, J. Chiang, S. Ciprini, R. Claus, J. Cohen-Tanugi, L. Costamante, S. Cutini, F. D'Ammando, C. D. Dermer, A. de Angelis, A. de Luca, F. de Palma, S. W. Digel, E. do Couto e Silva, P. S. Drell, A. DrlicaWagner, R. Dubois, D. Dumora, C. Favuzzi, S. J. Fegan, E. C. Ferrara, W. B. Focke, P. Fortin, M. Frailis, Y. Fukazawa, S. Funk, P. Fusco, F. Gargano, D. Gasparrini, N. Gehrels, S. Germani, N. Giglietto, F. Giordano, M. Giroletti, T. Glanzman, G. Godfrey, I. A. Grenier, M. H. Grondin, J. E. Grove, S. Guiriec, D. Hadasch, Y. Hanabata, A. K. Harding, K. Hayashi, M. Hayashida, E. Hays, D. Horan, R. Itoh, G. Jóhannesson, A. S. Johnson, T. J. Johnson, D. Khangulyan, T. Kamae, H. Katagiri, J. Kataoka, M. Kerr, J. Knödlseder, M. Kuss, J. Lande, L. Latronico, S. H. Lee, M. Lemoine-Goumard, F. Longo, F. Loparco, P. Lubrano, G. M. Madejski, A. Makeev, M. Marelli, M. N. Mazziotta, J. E. McEnery, P. F. Michelson, W. Mitthumsiri, T. Mizuno, A. A. Moiseev, C. Monte, M. E. Monzani, A. Morselli, I. V. Moskalenko, S. Murgia, T. Nakamori, M. NaumannGodo, P. L. Nolan, J. P. Norris, E. Nuss, T. Ohsugi, A. Okumura, N. Omodei, J. F. Ormes, M. Ozaki, D. Paneque, D. Parent, V. Pelassa, M. Pepe, M. PesceRollins, M. Pierbattista, F. Piron, T. A. Porter, S. Rainò, R. Rando, P. S. Ray, M. Razzano, A. Reimer, O. Reimer, T. Reposeur, S. Ritz, R. W. Romani, H. F. W. Sadrozinski, D. Sanchez, P. M. S. Parkinson, J. D. Scargle, T. L. Schalk, C. Sgrò, E. J. Siskind, P. D. Smith, G. Spand re, P. Spinelli, M. S. Strickman, D. J. Suson, H. Takahashi, T. Takahashi, T. Tanaka, J. B. Thayer, D. J. Thompson, L. Tibaldo, D. F. Torres, G. Tosti, A. Tramacere, E. Troja, Y. Uchiyama, J. Vandenbroucke, V. Vasileiou, G. Vianello, V. Vitale, P. Wang, K. S. Wood, Z. Yang, M. Ziegler, Gamma-Ray Flares from the Crab Nebula, Science 331 (6018) (2011) 739. arXiv: 1011.3855 , doi : 10.1126/science. 1199705 . 
[220] E. G. P. O'Connor, A. Shearer, C. Gouiffes, P. Laurent, High Time Resolution Astronomical Polarimetry with GASP, in: P. Weltevrede, B. B. P. Perera, L. L. Preston, S. Sanidas (Eds.), Pulsar Astrophysics the Next Fifty Years, Vol. 337 of IAU Symposium, 2018, pp. 384-385. doi : 10. 1017/S1743921317010626.

[221] S. V. Vadawale, T. Chattopadhyay, N. P. S. Mithun, A. R. Rao, D. Bhattacharya, A. Vibhute, V. B. Bhalerao, G. C. Dewangan, R. Misra, B. Paul, A. Basu, B. C. Joshi, S. Sreekumar, E. Samuel, P. Priya, P. Vinod, S. Seetha, Phase-resolved $\mathrm{X}$-ray polarimetry of the Crab pulsar with the AstroSat CZT Imager, Nature Astronomy 2 (2018) 50-55. doi : 10 . 1038/s41550-017-0293-z.

[222] E. Jourdain, J. P. Roques, 2003-2018 Monitoring of the Crab Nebula Polarization in Hard X-Rays with INTEGRAL SPI, ApJ, 882 (2) (2019) 129. arXiv: 1907. 09341, doi : 10.3847/1538-4357/ab3422. 
[223] A. A. Abdo, M. Ajello, A. Allafort, L. Baldini, J. Ballet, G. Barbiellini, M. G. Baring, D. Bastieri, A. Belfiore, R. Bellazzini, B. Bhattacharyya, E. Bissaldi, E. D. Bloom, E. Bonamente, E. Bottacini, T. J. Brandt, J. Bregeon, M. Brigida, P. Bruel, R. Buehler, M. Burgay, T. H. Burnett, G. Busetto, S. Buson, G. A. Caliandro, R. A. Cameron, F. Camilo, P. A. Caraveo, J. M. Casandjian, C. Cecchi, Ö. Çelik, E. Charles, S. Chaty, R. C. G. Chaves, A. Chekhtman, A. W. Chen, J. Chiang, G. Chiaro, S. Ciprini, R. Claus, I. Cognard, J. Cohen-Tanugi, L. R. Cominsky, J. Conrad, S. Cutini, F. D'Ammando, A. de Angelis, M. E. DeCesar, A. De Luca, P. R. den Hartog, F. de Palma, C. D. Dermer, G. Desvignes, S. W. Digel, L. Di Venere, P. S. Drell, A. Drlica-Wagner, R. Dubois, D. Dumora, C. M. Espinoza, L. Falletti, C. Favuzzi, E. C. Ferrara, W. B. Focke, A. Franckowiak, P. C. C. Freire, S. Funk, P. Fusco, F. Gargano, D. Gasparrini, S. Germani, N. Giglietto, P. Giommi, F. Giordano, M. Giroletti, T. Glanzman, G. Godfrey, E. V. Gotthelf, I. A. Grenier, M. H. Grondin, J. E. Grove, L. Guillemot, S. Guiriec, D. Hadasch, Y. Hanabata, A. K. Harding, M. Hayashida, E. Hays, J. Hessels, J. Hewitt, A. B. Hill, D. Horan, X. Hou, R. E. Hughes, M. S. Jackson, G. H. Janssen, T. Jogler, G. Jóhannesson, R. P. Johnson, A. S. Johnson, T. J. Johnson, W. N. Johnson, S. Johnston, T. Kamae, J. Kataoka, M. Keith, M. Kerr, J. Knödlseder, M. Kramer, M. Kuss, J. Lande, S. Larsson, L. Latronico, M. Lemoine-Goumard, F. Longo, F. Loparco, M. N. Lovellette, P. Lubrano, A. G. Lyne, R. N. Manchester, M. Marelli, F. Massaro, M. Mayer, M. N. Mazziotta, J. E. McEnery, M. A. McLaughlin, J. Mehault, P. F. Michelson, R. P. Mignani, W. Mitthumsiri, T. Mizuno, A. A. Moiseev, M. E. Monzani, A. Morselli, I. V. Moskalenko, S. Murgia, T. Nakamori, R. Nemmen, E. Nuss, M. Ohno, T. Ohsugi, M. Orienti, E. Orlando, J. F. Ormes, D. Paneque, J. H. Panetta, D. Parent, J. S. Perkins, M. Pesce-Rollins, M. Pierbattista, F. Piron, G. Pivato, H. J. Pletsch, T. A. Porter, A. Possenti, S. Rainò, R. Rando, S. M. Ransom, P. S. Ray, M. Razzano, N. Rea, A. Reimer, O. Reimer, N. Renault, T. Reposeur, S. Ritz, R. W. Romani, M. Roth, R. Rousseau, J. Roy, J. Ruan, A. Sartori, P. M. Saz Parkinson, J. D. Scargle, A. Schulz, C. Sgrò, R. Shannon, E. J. Siskind, D. A. Smith, G. Spandre, P. Spinelli, B. W. Stappers, A. W. Strong, D. J. Suson, H. Takahashi, J. G. Thayer, J. B. Thayer, G. Theureau, D. J. Thompson, S. E. Thorsett, L. Tibaldo, O. Tibolla, M. Tinivella, D. F. Torres, G. Tosti, E. Troja, Y. Uchiyama, T. L. Usher, J. Vandenbroucke, V. Vasileiou, C. Venter, G. Vianello, V. Vitale, N. Wang, P. Weltevrede, B. L. Winer, M. T. Wolff, D. L. Wood, K. S. Wood, M. Wood, Z. Yang, The Second Fermi Large Area Telescope Catalog of Gamma-Ray Pulsars, ApJS, 208 (2) (2013) 17. arXiv: 1305.4385, doi : 10.1088/0067-0049/208/2/17.

[224] S. V. Molkov, A. M. Cherepashchuk, A. A. Lutovinov, M. G. Revnivtsev, K. A. Postnov, R. A. Sunyaev, A Hard X-ray Survey of the Sagittarius Arm Tangent with the IBIS Telescope of the INTEGRAL Observatory: A Catalog of Sources, Astronomy Letters 30 (2004) 534-539. arXiv: astro-ph/0402416, doi : 10 . 1134/1.1784495.

[225] S. Keek, L. Kuiper, W. Hermsen, The discovery of five new hard X-ray sources 
in the Circinus region by INTEGRAL, The Astronomer's Telegram 810 (2006) 1.

[226] A. J. Bird, A. Bazzano, L. Bassani, F. Capitanio, M. Fiocchi, A. B. Hill, A. Malizia, V. A. McBride, S. Scaringi, V. Sguera, J. B. Stephen, P. Ubertini, A. J. Dean, F. Lebrun, R. Terrier, M. Renaud, F. Mattana, D. Götz, J. Rodriguez, G. Belanger, R. Walter, C. Winkler, The Fourth IBIS/ISGRI Soft Gamma-ray Survey Catalog, ApJS, 186 (1) (2010) 1-9. arXiv:0910.1704, doi : $10.1088 / 0067-0049 / 186 / 1 / 1$.

[227] E. V. Gotthelf, J. P. Halpern, R. Terrier, F. Mattana, Discovery of an Energetic 38.5 ms Pulsar Powering the Gamma-ray Source IGR J18490-0000/HESS J1849-000, ApJL, 729 (2) (2011) L16. arXiv: 1012.2121, doi:10.1088/ 2041-8205/729/2/L16.

[228] M. Renaud, V. Marandon, E. V. Gotthelf, J. Rodriguez, R. Terrier, F. Mattana, F. Lebrun, J. A. Tomsick, R. N. Manchester, Discovery of a Highly Energetic Pulsar Associated with IGR J14003-6326 in the Young Uncataloged Galactic Supernova Remnant G310.6-1.6, ApJ, 716 (1) (2010) 663-670. arXiv:0910. 3074, doi : 10.1088/0004-637X/716/1/663.

[229] L. Pavan, E. Bozzo, G. Pühlhofer, C. Ferrigno, M. Balbo, R. Walter, IGR J11014-6103: a newly discovered pulsar wind nebula?, A\&A, 533 (2011) A74. arXiv: 1107.2832, doi:10.1051/0004-6361/201117379.

[230] L. Pavan, P. Bordas, G. Pühlhofer, M. D. Filipović, A. De Horta, A. O’Brien, M. Balbo, R. Walter, E. Bozzo, C. Ferrigno, E. Crawford, L. Stella, The long helical jet of the Lighthouse nebula, IGR J11014-6103, A\&A, 562 (2014) A122. arXiv: 1309.6792, doi : 10.1051/0004-6361/201322588.

[231] J. P. Halpern, J. A. Tomsick, E. V. Gotthelf, F. Camilo, C. Y. Ng, A. Bodaghee, J. Rodriguez, S. Chaty, F. Rahoui, Discovery of X-Ray Pulsations from the INTEGRAL Source IGR J11014-6103, ApJL, 795 (2) (2014) L27. arXiv: 1410.2332, doi : 10.1088/2041-8205/795/2/L27.

[232] A. Malizia, L. Bassani, J. B. Stephen, A. Bazzano, P. Ubertini, A. J. Bird, A. J. Dean, V. Sguera, M. Renaud, R. Walter, F. Gianotti, The INTEGRAL/IBIS Source AX J1838.0-0655: A Soft X-Ray-to-TeV $\gamma$-Ray Broadband Emitter, ApJL, 630 (2) (2005) L157-L160. arXiv:astro-ph/0507660, doi: 10. $1086 / 491653$.

[233] F. Aharonian, A. G. Akhperjanian, K. M. Aye, A. R. Bazer-Bachi, M. Beilicke, W. Benbow, D. Berge, P. Berghaus, K. Bernlöhr, C. Boisson, O. Bolz, C. Borgmeier, I. Braun, F. Breitling, A. M. Brown, J. B. Gordo, P. M. Chadwick, L. M. Chounet, R. Cornils, L. Costamante, B. Degrange, A. Djannati-Ataï, L. O. Drury, G. Dubus, T. Ergin, P. Espigat, F. Feinstein, P. Fleury, G. Fontaine, S. Funk, Y. A. Gallant, B. Giebels, S. Gillessen, P. Goret, C. Hadjichristidis, M. Hauser, G. Heinzelmann, G. Henri, G. Hermann, J. A. Hinton, W. Hofmann, M. Holleran, D. Horns, O. C. de Jager, I. Jung, B. Khélifi, N. Komin, 
A. Konopelko, I. J. Latham, R. Le Gallou, A. Lemière, M. Lemoine, N. Leroy, T. Lohse, A. Marcowith, C. Masterson, T. J. L. McComb, M. de Naurois, S. J. Nolan, A. Noutsos, K. J. Orford, J. L. Osborne, M. Ouchrif, M. Panter, G. Pelletier, S. Pita, G. Pühlhofer, M. Punch, B. C. Raubenheimer, M. Raue, J. Raux, S. M. Rayner, I. Redondo, A. Reimer, O. Reimer, J. Ripken, L. Rob, L. Rolland, G. Rowell, V. Sahakian, L. Saugé, S. Schlenker, R. Schlickeiser, C. Schuster, U. Schwanke, M. Siewert, H. Sol, R. Steenkamp, C. Stegmann, J. P. Tavernet, R. Terrier, C. G. Théoret, M. Tluczykont, D. J. van der Walt, G. Vasileiadis, C. Venter, P. Vincent, B. Visser, H. J. Völk, S. J. Wagner, A New Population of Very High Energy Gamma-Ray Sources in the Milky Way, Science 307 (5717) (2005) 1938-1942. arXiv:astro-ph/0504380, doi : $10.1126 /$ science. 1108643 .

[234] E. V. Gotthelf, J. P. Halpern, F. Camilo, C. Markwardt, J. Swank, Discovery of a 70.5 ms Pulsar in AX J1838.0-0655 Associated with HESS J1837-069, The Astronomer's Telegram 1392 (2008) 1.

[235] L. Kuiper, W. Hermsen, M. Klein-Wolt, R. Wijnands, The spin-down rate, energetics and spectrum of the $70.5 \mathrm{~ms}$ pulsar in AX J1838.0-0655, The Astronomer's Telegram 1405 (2008) 1.

[236] L. Kuiper, W. Hermsen, High-energy characteristics of the schizophrenic pulsar PSR J1846-0258 in Kes 75. Multi-year RXTE and INTEGRAL observations crossing the magnetar-like outburst, A\&A, 501 (3) (2009) 1031-1046. arXiv: 0904 . 4376, doi : $10.1051 / 0004-6361 / 200811580$.

[237] H. S. Kumar, S. Safi-Harb, Variability of the High Magnetic Field X-Ray Pulsar PSR J1846-0258 Associated with the Supernova Remnant Kes 75 as Revealed by the Chandra X-Ray Observatory, ApJL, 678 (1) (2008) L43-L46. doi : 10. $1086 / 588284$.

[238] F. P. Gavriil, M. E. Gonzalez, E. V. Gotthelf, V. M. Kaspi, M. A. Livingstone, P. M. Woods, Magnetar-Like Emission from the Young Pulsar in Kes 75, Science 319 (5871) (2008) 1802. arXiv:0802 .1704, doi:10.1126/science. 1153465.

[239] L. Kuiper, W. Hermsen, A. Dekker, The Fermi-LAT detection of magnetar-like pulsar PSR J1846-0258 at high-energy gamma-rays, MNRAS, 475 (1) (2018) 1238-1250. arXiv: 1709.00899, doi : 10.1093/mnras/stx3128.

[240] F. Coti Zelati, D. F. Torres, J. Li, D. Viganò, Spectral characterization of the non-thermal X-ray emission of gamma-ray pulsars, MNRAS, 492 (1) (2020) 1025-1043. arXiv: 1912.03953, doi : 10.1093/mnras/stz3485.

[241] D. F. Torres, Order parameters for the high-energy spectra of pulsars, Nature Astronomy 2 (2018) 247-256. arXiv: 1802.04177, doi:10.1038/ s41550-018-0384-5. 
[242] D. F. Torres, D. Viganò, F. Coti Zelati, J. Li, Synchrocurvature modelling of the multifrequency non-thermal emission of pulsars, MNRAS, 489 (4) (2019) 5494-5512. arXiv: 1908.11574, doi : 10.1093/mnras/stz2403.

[252] S. Mereghetti, L. Stella, The Very Low Mass X-Ray Binary Pulsars: A New Class of Sources?, ApJL, 442 (1995) L17. doi : 10 . 1086/187805.

[253] S. Mereghetti, The strongest cosmic magnets: soft gamma-ray repeaters and anomalous X-ray pulsars, A\&AR, 15 (4) (2008) 225-287. arXiv:0804.0250, doi : $10.1007 / \mathrm{s} 00159-008-0011-z$.

[254] L. Kuiper, W. Hermsen, M. Mendez, Discovery of Hard Nonthermal Pulsed XRay Emission from the Anomalous X-Ray Pulsar 1E 1841-045, ApJ, 613 (2) (2004) 1173-1178. arXiv:astro-ph/0404582, doi : 10.1086/423129. 
[255] D. Götz, N. Rea, G. L. Israel, S. Zane, P. Esposito, E. V. Gotthelf, S. Mereghetti, A. Tiengo, R. Turolla, Long term hard X-ray variability of the anomalous X-ray pulsar 1RXS J170849.0-400910 discovered with INTEGRAL, A\&A, 475 (1) (2007) 317-321. arXiv:0709.3712, doi:10.1051/0004-6361:20078291.

[256] L. Kuiper, W. Hermsen, P. R. den Hartog, W. Collmar, Discovery of Luminous Pulsed Hard X-Ray Emission from Anomalous X-Ray Pulsars 1RXS J17084009, 4U 0142+61, and 1E 2259+586 by INTEGRAL and RXTE, ApJ, 645 (1) (2006) 556-575. arXiv: astro-ph/0603467, doi : 10 . 1086/504317.

[257] M. G. Revnivtsev, R. A. Sunyaev, D. A. Varshalovich, V. V. Zheleznyakov, A. M. Cherepashchuk, A. A. Lutovinov, E. M. Churazov, S. A. Grebenev, M. R. Gilfanov, A Hard X-ray Survey of the Galactic-Center Region with the IBIS Telescope of the INTEGRAL Observatory: A Catalog of Sources, Astronomy Letters 30 (2004) 382-389. arXiv:astro-ph/0402027, doi : $10.1134 / 1.1764884$.

[258] P. R. den Hartog, L. Kuiper, W. Hermsen, V. M. Kaspi, R. Dib, J. Knödlseder, F. P. Gavriil, Detailed high-energy characteristics of AXP 4U 0142+61. Multiyear observations with INTEGRAL, RXTE, XMM-Newton, and ASCA, A\&A, 489 (1) (2008) 245-261. arXiv:0804.1640, doi:10.1051/0004-6361: 200809390 .

[259] S. Mereghetti, D. Götz, I, F. Mirabel, K. Hurley, INTEGRAL discovery of persistent hard X-ray emission from the Soft Gamma-ray Repeater SGR 1806-20, A\&A, 433 (2) (2005) L9-L12. arXiv:astro-ph/0411695, doi:10.1051/ $0004-6361: 200500088$.

[260] S. Molkov, K. Hurley, R. Sunyaev, P. Shtykovsky, M. Revnivtsev, C. Kouveliotou, The broad-band spectrum of the persistent emission from SGR 1806-20, A\&A, 433 (2) (2005) L13-L16. arXiv:astro-ph/0411696, doi : 10 . 1051/ 0004-6361: 200500087.

[261] P. Esposito, S. Mereghetti, A. Tiengo, S. Zane, R. Turolla, D. Götz, N. Rea, N. Kawai, M. Ueno, G. L. Israel, L. Stella, M. Feroci, SGR 1806-20 about two years after the giant flare: Suzaku, XMM-Newton and INTEGRAL observations, A\&A, 476 (1) (2007) 321-330. arXiv:0710.2789, doi:10.1051/ 0004-6361:20078562.

[262] D. Götz, S. Mereghetti, A. Tiengo, P. Esposito, Magnetars as persistent hard X-ray sources: INTEGRAL discovery of a hard tail in SGR 1900+14, A\&A, 449 (2) (2006) L31-L34. arXiv: astro-ph/0602359, doi : 10 . 1051/ 0004-6361:20064870.

[263] L. Ducci, S. Mereghetti, D. Götz, A. Santangelo, Ten years of INTEGRAL observations of the hard X-ray emission from SGR 1900+14, A\&A, 583 (2015) A113. arXiv: 1510.00940 , doi : 10.1051/0004-6361/201527029. 
[264] N. Rea, G. L. Israel, R. Turolla, P. Esposito, S. Mereghetti, D. Götz, S. Zane, A. Tiengo, K. Hurley, M. Feroci, M. Still, V. Yershov, C. Winkler, R. Perna, F. Bernardini, P. Ubertini, L. Stella, S. Campana, M. van der Klis, P. Woods, The first outburst of the new magnetar candidate SGR0501+4516, MNRAS, 396 (4) (2009) 2419-2432. arXiv:0904 .2413, doi:10.1111/j.1365-2966.2009. 14920.x.

[265] F. Bernardini, G. L. Israel, L. Stella, R. Turolla, P. Esposito, N. Rea, S. Zane, A. Tiengo, S. Campana, D. Götz, S. Mereghetti, P. Romano, Multi-instrument $\mathrm{X}$-ray monitoring of the January 2009 outburst from the recurrent magnetar candidate 1E 1547.0-5408, A\&A, 529 (2011) A19. arXiv:1102.5419, doi : 10.1051/0004-6361/201016197.

[266] L. Kuiper, W. Hermsen, P. R. den Hartog, J. O. Urama, Temporal and Spectral Evolution in X-and $\gamma$-Rays of Magnetar 1E 1547.0-5408 since its 2008 October Outburst: The Discovery of a Transient Hard Pulsed Component after its 2009 January Outburst, ApJ, 748 (2) (2012) 133. arXiv: 1201 . 5530, doi : 10 . 1088/ 0004-637X/748/2/133.

[267] T. Enoto, N. Rea, Y. E. Nakagawa, K. Makishima, T. Sakamoto, P. Esposito, D. Götz, K. Hurley, G. L. Israel, M. Kokubun, S. Mereghetti, H. Murakami, K. Nakazawa, L. Stella, A. Tiengo, R. Turolla, S. Yamada, K. Yamaoka, A. Yoshida, S. Zane, Wide-band Suzaku Analysis of the Persistent Emission from SGR 0501+4516 During the 2008 Outburst, ApJ, 715 (1) (2010) 665-670. doi : $10.1088 / 0004-637 \mathrm{X} / 715 / 1 / 665$.

[268] T. Enoto, S. Shibata, T. Kitaguchi, Y. Suwa, T. Uchide, H. Nishioka, S. Kisaka, T. Nakano, H. Murakami, K. Makishima, Magnetar Broadband X-Ray Spectra Correlated with Magnetic Fields: Suzaku Archive of SGRs and AXPs Combined with NuSTAR, Swift, and RXTE, ApJS, 231 (1) (2017) 8. arXiv : 1704.07018, doi : 10.3847/1538-4365/aa6f0a.

[269] H. An, V. M. Kaspi, R. Archibald, M. Bachetti, V. Bhalerao, E. C. Bellm, A. M. Beloborodov, S. E. Boggs, D. Chakrabarty, F. E. Christensen, W. W. Craig, F. Dufour, K. Forster, E. V. Gotthelf, B. W. Grefenstette, C. J. Hailey, F. A. Harrison, R. Hascoët, T. Kitaguchi, C. Kouveliotou, K. K. Madsen, K. Mori, M. J. Pivovaroff, V. R. Rana, D. Stern, S. Tendulkar, J. A. Tomsick, J. K. Vogel, W. W. Zhang, NuSTAR Team, NuSTAR results and future plans for magnetar and rotation-powered pulsar observations, Astronomische Nachrichten 335 (3) (2014) 280-284. arXiv: 1402 .1079, doi:10.1002/asna. 201312032.

[270] P. R. den Hartog, L. Kuiper, W. Hermsen, Detailed high-energy characteristics of AXP 1RXS J170849-400910. Probing the magnetosphere using INTEGRAL, RXTE, and XMM-Newton, A\&A, 489 (1) (2008) 263-279. arXiv : 0804 . 1641, doi : $10.1051 / 0004-6361: 200809772$.

[271] A. M. Beloborodov, On the Mechanism of Hard X-Ray Emission from Magnetars, ApJ, 762 (1) (2013) 13. arXiv: 1201.0664, doi:10.1088/0004-637X/ 762/1/13. 
[272] M. G. Baring, A. K. Harding, Resonant Compton upscattering in anomalous Xray pulsars, Ap\&SS, 308 (1-4) (2007) 109-118. arXiv: astro-ph/0610382, doi : $10.1007 / \mathrm{s} 10509-007-9326-\mathrm{x}$

[273] S. Zane, R. Turolla, L. Nobili, N. Rea, Modeling the broadband persistent emission of magnetars, Advances in Space Research 47 (8) (2011) 1298-1304. arXiv: 1008.1537 , doi : 10.1016/j.asr .2010.08.003.

[274] A. M. Beloborodov, Electron-Positron Flows around Magnetars, ApJ, 777 (2) (2013) 114. arXiv: 1209.4063, doi : 10.1088/0004-637X/777/2/114.

[275] Z. Wadiasingh, M. G. Baring, P. L. Gonthier, A. K. Harding, Resonant Inverse Compton Scattering Spectra from Highly Magnetized Neutron Stars, ApJ, 854 (2) (2018) 98. arXiv: 1712 . 09643, doi : 10 . 3847/1538-4357/aaa460.

[276] R. Hascoët, A. M. Beloborodov, P. R. den Hartog, Phase-resolved X-Ray Spectra of Magnetars and the Coronal Outflow Model, ApJL, 786 (1) (2014) L1. arXiv: 1401.3406 , doi : $10.1088 / 2041-8205 / 786 / 1 / \mathrm{L} 1$.

[277] S. P. Tendulkar, R. Hascöet, C. Yang, V. M. Kaspi, A. M. Beloborodov, H. An, M. Bachetti, S. E. Boggs, F. E. Christensen, W. W. Craig, S. Guillot, C. A. Hailey, F. A. Harrison, D. Stern, W. Zhang, Phase-resolved NuSTAR and SwiftXRT Observations of Magnetar 4U 0142+61, ApJ, 808 (1) (2015) 32. arXiv: 1506.03098, doi : $10.1088 / 0004-637 \mathrm{X} / 808 / 1 / 32$.

[278] S. Mereghetti, D. Götz, J. Borkowski, R. Walter, H. Pedersen, The INTEGRAL Burst Alert System, A\&A, 411 (2003) L291-L297. arXiv: astro-ph/ 0308173, doi : 10.1051/0004-6361:20031289.

[279] S. Mereghetti, V. Savchenko, C. Ferrigno, D. Götz, M. Rigoselli, A. Tiengo, A. Bazzano, E. Bozzo, A. Coleiro, T. J. L. Courvoisier, M. Doyle, A. Goldwurm, L. Hanlon, E. Jourdain, A. von Kienlin, A. Lutovinov, A. Martin-Carrillo, S. Molkov, L. Natalucci, F. Onori, F. Panessa, J. Rodi, J. Rodriguez, C. SánchezFernández, R. Sunyaev, P. Ubertini, INTEGRAL Discovery of a Burst with Associated Radio Emission from the Magnetar SGR 1935+2154, ApJL, 898 (2) (2020) L29. arXiv: 2005.06335, doi : 10.3847/2041-8213/aba2cf.

[280] The CHIME/FRB Collaboration, :, B. C. Andersen, K. M. Band ura, M. Bhardwaj, A. Bij, M. M. Boyce, P. J. Boyle, C. Brar, T. Cassanelli, P. Chawla, T. Chen, J. F. Cliche, A. Cook, D. Cubranic, A. P. Curtin, N. T. Denman, M. Dobbs, F. Q. Dong, M. Fandino, E. Fonseca, B. M. Gaensler, U. Giri, D. C. Good, M. Halpern, A. S. Hill, G. F. Hinshaw, C. Höfer, A. Josephy, J. W. Kania, V. M. Kaspi, T. L. Landecker, C. Leung, D. Z. Li, H. H. Lin, K. W. Masui, R. Mckinven, J. Mena-Parra, M. Merryfield, B. W. Meyers, D. Michilli, N. Milutinovic, A. Mirhosseini, M. Münchmeyer, A. Naidu, L. B. Newburgh, C. Ng, C. Patel, U. L. Pen, T. Pinsonneault-Marotte, Z. Pleunis, B. M. Quine, M. Rafiei-Ravandi, M. Rahman, S. M. Ransom, A. Renard, P. Sanghavi, P. Scholz, J. R. Shaw, K. Shin, S. R. Siegel, S. Singh, R. J. Smegal, K. M. Smith, I. H. Stairs, C. M. Tan, S. P. Tendulkar, I. Tretyakov, K. Vanderlinde, H. Wang, D. Wulf, A. V. 
Zwaniga, A bright millisecond-duration radio burst from a Galactic magnetar, arXiv e-prints (2020) arXiv:2005.10324arXiv:2005.10324.

[281] C. D. Bochenek, V. Ravi, K. V. Belov, G. Hallinan, J. Kocz, S. R. Kulkarni, D. L. McKenna, A fast radio burst associated with a Galactic magnetar, arXiv e-prints (2020) arXiv:2005.10828arXiv:2005. 10828.

[282] D. Götz, S. Mereghetti, I. F. Mirabel, K. Hurley, Spectral evolution of weak bursts from SGR 1806-20 observed with INTEGRAL, A\&A, 417 (2004) L45L48. arXiv: astro-ph/0403018, doi : 10. 1051/0004-6361:20040080.

[283] D. Götz, S. Mereghetti, S. Molkov, K. Hurley, I. F. Mirabel, R. Sunyaev, G. Weidenspointner, S. Brand t, M. del Santo, M. Feroci, E. Gögüs, A. von Kienlin, M. van der Klis, C. Kouveliotou, N. Lund, G. Pizzichini, P. Ubertini, C. Winkler, P. M. Woods, Two years of INTEGRAL monitoring of the soft gamma-ray repeater SGR 1806-20: from quiescence to frenzy, A\&A, 445 (1) (2006) 313321. arXiv: astro-ph/0508615, doi : 10.1051/0004-6361:20053648.

[284] S. Mereghetti, D. Götz, G. Weidenspointner, A. von Kienlin, P. Esposito, A. Tiengo, G. Vianello, G. L. Israel, L. Stella, R. Turolla, N. Rea, S. Zane, Strong Bursts from the Anomalous X-Ray Pulsar 1E 1547.0-5408 Observed with the INTEGRAL/SPI Anti-Coincidence Shield, ApJL, 696 (1) (2009) L74L78. arXiv: 0903 . 1974, doi : 10.1088/0004-637X/696/1/L74.

[285] V. Savchenko, A. Neronov, V. Beckmann, N. Produit, R. Walter, SGR-like flaring activity of the anomalous X-ray pulsar 1E 1547.0-5408, A\&A, 510 (2010) A77. arXiv:0912.0290, doi : 10.1051/0004-6361/200911988.

[286] A. Tiengo, G. Vianello, P. Esposito, S. Mereghetti, A. Giuliani, E. Costantini, G. L. Israel, L. Stella, R. Turolla, S. Zane, N. Rea, D. Götz, F. Bernardini, A. Moretti, P. Romano, M. Ehle, N. Gehrels, The Dust-scattering X-ray Rings of the Anomalous X-ray Pulsar 1E 1547.0-5408, ApJ, 710 (1) (2010) 227-235. arXiv:0911.3064, doi : 10.1088/0004-637X/710/1/227.

[287] C. K. Li, L. Lin, S. L. Xiong, M. Y. Ge, X. B. Li, T. P. Li, F. J. Lu, S. N. Zhang, Y. L. Tuo, Y. Nang, B. Zhang, S. Xiao, Y. Chen, L. M. Song, Y. P. Xu, C. Z. Liu, S. M. Jia, X. L. Cao, S. Zhang, J. L. Qu, J. Y. Liao, X. F. Zhao, Y. Tan, J. Y. Nie, H. S. Zhao, S. J. Zheng, Y. G. Zheng, Q. Luo, C. Cai, B. Li, W. C. Xue, Q. C. Bu, Z. Chang, G. Chen, L. Chen, T. X. Chen, Y. B. Chen, Y. P. Chen, W. Cui, W. W. Cui, J. K. Deng, Y. W. Dong, Y. Y. Du, M. X. Fu, G. H. Gao, H. Gao, M. Gao, Y. D. Gu, J. Guan, C. C. Guo, D. W. Han, Y. Huang, J. Huo, L. H. Jiang, W. C. Jiang, J. Jin, Y. J. Jin, L. D. Kong, G. Li, M. S. Li, W. Li, X. Li, X. F. Li, Y. G. Li, Z. W. Li, X. H. Liang, B. S. Liu, G. Q. Liu, H. W. Liu, X. J. Liu, Y. N. Liu, B. Lu, X. F. Lu, T. Luo, X. Ma, B. Meng, G. Ou, N. Sai, R. C. Shang, X. Y. Song, L. Sun, L. Tao, C. Wang, G. F. Wang, J. Wang, W. S. Wang, Y. S. Wang, X. Y. Wen, B. B. Wu, B. Y. Wu, M. Wu, G. C. Xiao, J. W. Yang, S. Yang, Y. J. Yang, Y.-J. Yang, Q. B. Yi, Q. Q. Yin, Y. You, A. M. Zhang, C. M. Zhang, F. Zhang, H. M. Zhang, J. Zhang, T. Zhang, 
W. Zhang, W. C. Zhang, W. Z. Zhang, Y. Zhang, Y. Zhang, Y. F. Zhang, Y. J. Zhang, Z. Zhang, Z. Zhang, Z. L. Zhang, D. K. Zhou, J. F. Zhou, Y. Zhu, Y. X. Zhu, R. L. Zhuang, Identification of a non-thermal X-ray burst with the Galactic magnetar SGR 1935+2154 and a fast radio burst with Insight-HXMT, arXiv e-prints (2020) arXiv:2005.11071arXiv:2005 . 11071.

[288] A. Ridnaia, D. Svinkin, D. Frederiks, A. Bykov, S. Popov, R. Aptekar, S. Golenetskii, A. Lysenko, A. Tsvetkova, M. Ulanov, T. Cline, A peculiar hard X-ray counterpart of a Galactic fast radio burst, arXiv e-prints (2020) arXiv:2005.11178arXiv:2005.11178.

[289] E. Petroff, J. W. T. Hessels, D. R. Lorimer, Fast radio bursts, A\&AR, 27 (1) (2019) 4. arXiv: 1904.07947, doi : 10.1007/s00159-019-0116-6.

[290] E. Platts, A. Weltman, A. Walters, S. P. Tendulkar, J. E. B. Gordin, S. Kandhai, A living theory catalogue for fast radio bursts, 821 (2019) 1-27. arXiv: 1810 . 05836, doi : 10.1016/j . physrep. 2019.06.003.

[291] J. Borkowski, D. Gotz, S. Mereghetti, N. Mowlavi, S. Shaw, M. Turler, Giant flare from Sgr 1806-20 detected by INTEGRAL., GRB Coordinates Network 2920 (2004) 1.

[292] S. Mereghetti, A. Tiengo, P. Esposito, D. Götz, L. Stella, G. L. Israel, N. Rea, M. Feroci, R. Turolla, S. Zane, An XMM-Newton View of the Soft Gamma Repeater SGR 1806-20: Long-Term Variability in the Pre-Giant Flare Epoch, ApJ, 628 (2) (2005) 938-945. arXiv:astro-ph/0502417, doi:10.1086/ 430943.

[293] J. L. Bibby, P. A. Crowther, J. P. Furness, J. S. Clark, A downward revision to the distance of the 1806-20 cluster and associated magnetar from Gemini NearInfrared Spectroscopy, MNRAS, 386 (1) (2008) L23-L27. arXiv: 0802 . 0815 , doi : $10.1111 / \mathrm{j} .1745-3933.2008 .00453 . \mathrm{x}$.

[294] S. Mereghetti, D. Götz, A. von Kienlin, A. Rau, G. Lichti, G. Weidenspointner, P. Jean, The First Giant Flare from SGR 1806-20: Observations Using the Anticoincidence Shield of the Spectrometer on INTEGRAL, ApJL, 624 (2) (2005) L105-L108. arXiv: astro-ph/0502577, doi : 10 . 1086/430669.

[295] D. M. Palmer, S. Barthelmy, N. Gehrels, R. M. Kippen, T. Cayton, C. Kouveliotou, D. Eichler, R. A. M. J. Wijers, P. M. Woods, J. Granot, Y. E. Lyubarsky, E. Ramirez-Ruiz, L. Barbier, M. Chester, J. Cummings, E. E. Fenimore, M. H. Finger, B. M. Gaensler, D. Hullinger, H. Krimm, C. B. Markwardt, J. A. Nousek, A. Parsons, S. Patel, T. Sakamoto, G. Sato, M. Suzuki, J. Tueller, A giant $\gamma$-ray flare from the magnetar SGR 1806 - 20, Nat., 434 (7037) (2005) 1107-1109. arXiv: astro-ph/0503030, doi : 10.1038/nature03525.

[296] T. Terasawa, Y. T. Tanaka, Y. Takei, N. Kawai, A. Yoshida, K. Nomoto, I. Yoshikawa, Y. Saito, Y. Kasaba, T. Takashima, T. Mukai, H. Noda, T. Murakami, K. Watanabe, Y. Muraki, T. Yokoyama, M. Hoshino, Repeated injections of energy in the first $600 \mathrm{~ms}$ of the giant flare of SGR1806 - 20, Nat., 
[297] K. Hurley, S. E. Boggs, D. M. Smith, R. C. Duncan, R. Lin, A. Zoglauer, S. Krucker, G. Hurford, H. Hudson, C. Wigger, W. Hajdas, C. Thompson, I. Mitrofanov, A. Sanin, W. Boynton, C. Fellows, A. von Kienlin, G. Lichti, A. Rau, T. Cline, An exceptionally bright flare from SGR 1806-20 and the origins of short-duration $\gamma$-ray bursts, Nat., 434 (7037) (2005) 1098-1103. arXiv: astro-ph/0502329, doi : 10.1038/nature03519.

[298] D. D. Frederiks, V. D. Palshin, R. L. Aptekar, S. V. Golenetskii, T. L. Cline, E. P. Mazets, On the possibility of identifying the short hard burst GRB 051103 with a giant flare from a soft gamma repeater in the M81 group of galaxies, Astronomy Letters 33 (1) (2007) 19-24. arXiv:astro-ph/0609544, doi: 10.1134/S1063773707010021.

[299] S. E. Boggs, A. Zoglauer, E. Bellm, K. Hurley, R. P. Lin, D. M. Smith, C. Wigger, W. Hajdas, The Giant Flare of 2004 December 27 from SGR 1806-20, ApJ, 661 (1) (2007) 458-467. arXiv : astro-ph/0611318, doi : 10.1086/516732.

[300] J. Granot, E. Ramirez-Ruiz, G. B. Taylor, D. Eichler, Y. E. Lyubarsky, R. A. M. J. Wijers, B. M. Gaensler, J. D. Gelfand, C. Kouveliotou, Diagnosing the Outflow from the SGR 1806-20 Giant Flare with Radio Observations, ApJ, 638 (1) (2006) 391-396. arXiv:astro-ph/0503251, doi : 10.1086/497680.

[301] M. J. Middleton, P. Casella, P. Gandhi, E. Bozzo, G. Anderson, N. Degenaar, I. Donnarumma, G. Israel, C. Knigge, A. Lohfink, S. Markoff, T. Marsh, N. Rea, S. Tingay, K. Wiersema, D. Altamirano, D. Bhattacharya, W. N. Brandt, S. Carey, P. Charles, M. Díaz Trigo, C. Done, M. Kotze, S. Eikenberry, R. Fender, P. Ferruit, F. Fürst, J. Greiner, A. Ingram, L. Heil, P. Jonker, S. Komossa, B. Leibundgut, T. Maccarone, J. Malzac, V. McBride, J. MillerJones, M. Page, E. M. Rossi, D. M. Russell, T. Shahbaz, G. R. Sivakoff, M. Tanaka, D. J. Thompson, M. Uemura, P. Uttley, G. van Moorsel, M. van Doesburgh, B. Warner, B. Wilkes, J. Wilms, P. Woudt, Paving the way to simultaneous multi-wavelength astronomy, New. Astron. Rev., 79 (2017) 26-48. arXiv: 1709.03520 , doi : $10.1016 / j$.newar . 2017.07.002. 


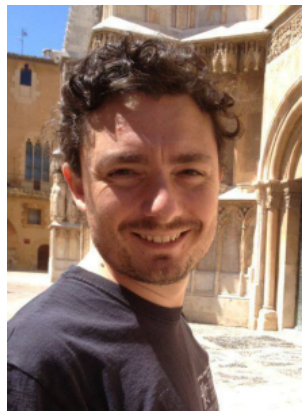

A. Papitto Master degree in Physics (2005) and PhD in Astronomy (2009) at the University of Roma Tor Vergata . Young researcher in Sardinia fellow at the University of Cagliari (2009-11), Juan de la Cierva fellow at the Institute of Space Sciences of Barcelona (2012-15), Marie Skodowska Curie Individual fellow at the INAF Astronomical Observatory of Rome (2016-2018) and currently permanent researcher at the same institute. Main research interests are in multiwavelength (mainly X-ray) observations of millisecond pulsars in binary systems, with a focus on transitional systems. He is co-PI of the fast photometer SiFAP2 for optical high time resolution astronomy. 